\title{
Using the Zeldovich dynamics to test expansion schemes
}

\author{
P. Valageas \\ Service de Physique Théorique, CEA Saclay, 91191 Gif-sur-Yvette, France \\ e-mail: volag@spht.saclay.cea.fr
}

Received 12 June 2007 / Accepted 24 August 2007

\begin{abstract}
Aims. We apply various expansion schemes that may be used to study gravitational clustering to the simple case of the Zeldovich dynamics.

Methods. Using the well-known exact solution of the Zeldovich dynamics we can compare the predictions of these various perturbative methods with the exact nonlinear result. We can also study their convergence properties and their behavior at high orders.

Results. We find that most systematic expansions fail to recover the decay of the response function in the highly nonlinear regime. "Linear methods" lead to increasingly fast growth in the nonlinear regime for higher orders, except for Padé approximants that give a bounded response at any order. "Nonlinear methods" manage to obtain some damping at one-loop order but they fail at higher orders. Although it recovers the exact Gaussian damping, a resummation in the high- $k$ limit is not justified very well as the generation of nonlinear power does not originate from a finite range of wavenumbers (hence there is no simple separation of scales). No method is able to recover the relaxation of the matter power spectrum on highly nonlinear scales. It is possible to impose a Gaussian cutoff in a somewhat ad-hoc fashion to reproduce the behavior of the exact two-point functions for two different times. However, this cutoff is not directly related to the clustering of matter and disappears in exact equal-time statistics such as the matter power spectrum. On a quantitative level, on weakly nonlinear scales, the usual perturbation theory, and the nonlinear scheme to which one adds an ansatz for the response function with such a Gaussian cutoff, are the two most efficient methods. We can expect these results to hold for the gravitational dynamics as well (this has been explicitly checked at one-loop order), since the structure of the equations of motion is identical for both dynamics.
\end{abstract}

Key words. gravitation - cosmology: theory - large-scale structure of Universe

\section{Introduction}

The growth of large-scale structures in the Universe through the amplification of small primordial fluctuations by gravitational instability is a key ingredient of modern cosmology (Peebles 1980). This process can be used to constrain cosmological parameters through the dependence of the matter power spectrum on scale and redshift. For instance, observations of highly nonlinear objects such as galaxy clusters can help constrain the normalization of the matter power spectrum and the average matter density (Oukbir \& Blanchard 1992; Younger et al. 2005). Although theoretical predictions are not very accurate on these small scales, one can still derive useful constraints because they are very rare objects, so that their dependence on cosmological parameters is very steep. Alternative probes, such as baryonic acoustic oscillations (Eisenstein et al. 1998, 2005) or weak lensing surveys (Munshi et al. 2007; Massey et al. 2007), focus on weakly nonlinear scales where they aim at measuring the matter distribution through its power spectrum or its two-point correlation, which are sensitive to typical fluctuations. In such cases, one needs an accurate theoretical prediction to derive useful constraints on cosmology. This problem is usually tackled through $\mathrm{N}$-body simulations or perturbative expansions for scales not too far from the linear regime (Bernardeau et al. 2002). However, numerical simulations are rather costly and analytical methods may have the advantage of leading to a better understanding of the physics at work. Moreover, on such large scales, a hydrodynamical description should be sufficient (i.e. one neglects shell crossing). This facilitates analytical approaches as one can use the continuity and Euler equations instead of the Vlasov equation. Therefore, there has recently been renewed interest in perturbation theory to devise analytical methods that could exhibit good accuracy on weakly nonlinear scales to be used for such observational probes (Crocce \& Scoccimarro 2006a,b; Valageas 2007; McDonald 2007; Matarrese \& Pietroni 2007a,b).

Thus, Crocce \& Scoccimarro (2006a,b) find that one can perform a partial resummation of the diagrammatic series that appears in the standard perturbative expansion to obtain a response function that decays into the nonlinear regime as expected (whereas the standard expansion grows as a polynomial of increasing order as we truncate the series at higher order). Moreover, this result agrees well with numerical simulations and can be used as an intermediate tool for obtaining a more accurate prediction of the matter power spectrum than with the usual perturbation theory (Crocce \& Scoccimarro 2007). On the other hand, Valageas (2007) present a path-integral formalism, so that the system is fully defined by its action $S$ (or its partition function $Z$ ). Then, one can apply the usual tools of field theory, such as large- $N$ expansions (similar to a semi-classical expansion over powers of $\hbar$ or a generalization to $N$ fields), to derive the matter power spectrum (Valageas 2007). Note that this method also applies to the highly nonlinear scales described by the Vlasov equation (Valageas 2004). Next, Matarrese \& Pietroni (2007a) have recently proposed an alternative method based on the path-integral formalism where one considers the dependence of the system on a large-wavenumber cutoff $\Lambda$. This gives rise to a new set of equations and, by taking the limit 
$\Lambda \rightarrow \infty$, one recovers the original system. These various methods may be seen as different reorganizations of the standard perturbative expansion. Of course, they all involve some truncation at some stage (otherwise the problem would be solved exactly), and they are all consistent up to that order (i.e. they only differ by higher-order terms).

To check the range of validity of such expansions, one must compare their predictions with $N$-body simulations (and assume for observational purposes that the accuracy remains the same for close cosmological parameters). This is not very convenient, since simulations themselves may be of limited accuracy. Along with this problem, the behaviors of other two-point functions than the power spectrum $P(k)$, such as the response function and self-energies and especially different-time functions such as $\left\langle\delta\left(\boldsymbol{x}_{1}, t_{1}\right) \delta\left(\boldsymbol{x}_{2}, t_{2}\right)\right\rangle$ with $t_{1} \neq t_{2}$, have not been analyzed in detail from $N$-body simulations (which do not give direct access to self-energies either). Therefore, it is interesting to investigate these theoretical methods applied to a simpler dynamics that can be solved exactly. Then, one can do a detailed comparison of the predictions of various expansion schemes with the exact nonlinear results. Moreover, one can reconstruct such expansion schemes in a direct manner from the exact two-point functions without computing high-order diagrams that involve many integrals, simply by expanding back the exact nonlinear result. In this way one can more easily investigate the convergence properties of these expansions and the behavior of high-order terms. A simple dynamics that can be solved exactly but that remains close to the gravitational dynamics (at least up to weakly nonlinear scales) is provided by the Zeldovich approximation (Zeldovich 1970; Gurbatov et al. 1989; Shandarin \& Zeldovich 1989). The latter was originally devised as an approximation to the gravitational dynamics. Here we take a different point of view as we modify the equations of motion so that the system is exactly given by the simple Zeldovich dynamics. Then, we apply to these new equations of motion various methods which can be applied to both dynamics (and to other stochastic dynamics such as the Navier-Stokes equations). Taking advantage of the exact results which can be obtained for the Zeldovich dynamics and its simpler properties we study the accuracy and the general properties of these expansion schemes in detail. This should shed light on the behavior of these methods applied to the gravitational dynamics, because both dynamics exhibit similar equations of motion, and these expansions apply in identical manner to both systems.

This article is organized as follows. First, in Sect. 2 we derive the equations of motion associated with the Zeldovich dynamics and their linear solution. Next, in Sect. 3 we obtain the path-integral formulation of this system, starting from either the differential form or the integral form of the equations of motion, in order to make the connection with the different approaches used in the literature. Then, we briefly describe how some expansion schemes can be built from this path-integral formalism, such as the large- $N$ expansions in Sect. 4 and the evolution equations with respect to a high- $k$ cutoff in Sect. 5. Before investigating such methods, we first derive the exact nonlinear two-point functions which can be obtained from the well-known exact solution of the Zeldovich dynamics in Sect. 6. Then, we describe the behavior of the standard perturbation theory in Sect. 7 and of the steepest-descent method (built from a large- $N$ expansion) in Sect. 8. Next, we discuss in Sect. 9 the high- $k$ resummation proposed by Crocce \& Scoccimarro (2006b) to improve the behavior of such expansion schemes. We turn to the 2PI effective action method in Sect. 10 (a second approach built from a large- $N$ expansion) and to simple nonlinear schemes associated with this expansion in Sect. 11. We investigate simple nonlinear schemes associated with the dependence on a high- $k$ cutoff in Sect. 12. Finally, in Sect. 13 we study the quantitative predictions on weakly nonlinear scales of these methods at one-loop order and we conclude in Sect. 14.

\section{Equations of motion}

\subsection{Zeldovich approximation}

On scales much larger than the Jeans length, both the cold dark matter and the baryons can be described as a pressureless dust. Then, we can neglect orbit crossings and use a hydrodynamical description governed by the equations of motion (Peebles 1980):

$\frac{\partial \delta}{\partial \tau}+\nabla \cdot[(1+\delta) v]=0$

$\frac{\partial v}{\partial \tau}+\mathcal{H} \boldsymbol{v}+(\boldsymbol{v} \cdot \nabla) \boldsymbol{v}=-\nabla \phi$

$\Delta \phi=\frac{3}{2} \Omega_{\mathrm{m}} \mathcal{H}^{2} \delta$

where $\tau=\int \mathrm{d} t / a$ is the conformal time (and $a$ the scale factor), $\mathcal{H}=\mathrm{d} \ln a / \mathrm{d} \tau$ the conformal expansion rate, and $\Omega_{\mathrm{m}}$ the matter density cosmological parameter. Here, $\delta$ is the matter density contrast and $v$ the peculiar velocity. Since the vorticity field decays within linear theory (Peebles 1980), we take the velocity to be a potential field so that $v$ is fully specified by its divergence $\theta$ or by its potential $\chi$ with

$\theta=\nabla \cdot \boldsymbol{v}, \quad \boldsymbol{v}=-\nabla \chi \quad$ whence $\theta=-\Delta \chi$

In the linear regime, one finds that the linear growing mode satisfies

$\theta_{\mathrm{L}}=-f \mathcal{H} \delta_{\mathrm{L}} \quad$ whence $\quad \phi_{\mathrm{L}}=\frac{3 \Omega_{\mathrm{m}} \mathcal{H}}{2 f} \chi_{\mathrm{L}}$,

where $f(\tau)$ is defined from the linear growing rate $D_{+}(\tau)$ of the density contrast by

$f=\frac{\mathrm{d} \ln D_{+}}{\mathrm{d} \ln a}=\frac{1}{\mathcal{H}} \frac{\mathrm{d} \ln D_{+}}{\mathrm{d} \tau}$,

and $D_{+}(\tau)$ is the growing solution of

$\frac{\mathrm{d}^{2} D_{+}}{\mathrm{d} \tau^{2}}+\mathcal{H} \frac{\mathrm{d} D_{+}}{\mathrm{d} \tau}=\frac{3}{2} \Omega_{\mathrm{m}} \mathcal{H}^{2} D_{+}$.

If we make the approximation that relation (5) remains valid in the nonlinear regime, that is, we replace the Poisson Eq. (3) by the second Eq. (5): $\phi=3 \Omega_{\mathrm{m}} \mathcal{H} \chi / 2 f$, then we obtain for the Euler Eq. (2):

$\frac{\partial v}{\partial \tau}+\left(1-\frac{3}{2} \frac{\Omega_{\mathrm{m}}}{f}\right) \mathcal{H} \boldsymbol{v}+(\boldsymbol{v} \cdot \nabla) \boldsymbol{v}=0$

Obviously, as shown by Eq. (8), within this approximation the velocity field now evolves independently of the density field. As is well known (Gurbatov et al. 1989), approximation (8) is actually identical to the Zeldovich approximation. Indeed, a change of variables for the velocity field yields

$\frac{\partial \boldsymbol{u}}{\partial D_{+}}+(\boldsymbol{u} \cdot \nabla) \boldsymbol{u}=0 \quad$ with $\quad \boldsymbol{v}=\left(\frac{\mathrm{d} D_{+}}{\mathrm{d} \tau}\right) \boldsymbol{u}$ 
Equation (9) is the equation of motion of free particles, $\mathrm{d} \boldsymbol{u} / \mathrm{d} D_{+}=0$, hence the trajectories are given by

$\boldsymbol{x}=\boldsymbol{q}+D_{+}(\tau) \boldsymbol{s}_{\mathrm{L} 0}(\boldsymbol{q}), \quad \boldsymbol{v}=\frac{\mathrm{d} D_{+}}{\mathrm{d} \tau} s_{\mathrm{L} 0}(\boldsymbol{q})$,

where $\boldsymbol{q}$ is the Lagrangian coordinate and $\boldsymbol{s}=\boldsymbol{s}_{\mathrm{L}}=D_{+} \boldsymbol{s}_{\mathrm{L} 0}$ is the displacement field that is exactly given by the linear theory. Equation (10) is the usual definition of the Zeldovich approximation (i.e. setting $s=s_{\mathrm{L}}$ ).

Thus, the Zeldovich approximation corresponds to a change in the linear term of the Euler equation, keeping the quadratic term and the continuity equation unchanged. Therefore, the analysis presented in Valageas (2007) for the case of the exact gravitational dynamics applies to the Zeldovich dynamics up to minor modifications. First, the equations of motion (1) and (8) read in Fourier space as

$$
\begin{aligned}
\frac{\partial \delta(\boldsymbol{k}, \tau)}{\partial \tau}+\theta(\boldsymbol{k}, \tau)= & -\int \mathrm{d} \boldsymbol{k}_{1} \mathrm{~d} \boldsymbol{k}_{2} \delta_{\mathrm{D}}\left(\boldsymbol{k}_{1}+\boldsymbol{k}_{2}-\boldsymbol{k}\right) \\
& \times \alpha\left(\boldsymbol{k}_{1}, \boldsymbol{k}_{2}\right) \theta\left(\boldsymbol{k}_{1}, \tau\right) \delta\left(\boldsymbol{k}_{2}, \tau\right)
\end{aligned}
$$

$$
\begin{aligned}
& \frac{\partial \theta(\boldsymbol{k}, \tau)}{\partial \tau}+\left(1-\frac{3 \Omega_{\mathrm{m}}}{2 f}\right) \mathcal{H} \theta(\boldsymbol{k}, \tau)= \\
& -\int \mathrm{d} \boldsymbol{k}_{1} \mathrm{~d} \boldsymbol{k}_{2} \delta_{\mathrm{D}}\left(\boldsymbol{k}_{1}+\boldsymbol{k}_{2}-\boldsymbol{k}\right) \beta\left(\boldsymbol{k}_{1}, \boldsymbol{k}_{2}\right) \theta\left(\boldsymbol{k}_{1}, \tau\right) \theta\left(\boldsymbol{k}_{2}, \tau\right)
\end{aligned}
$$

where $\delta_{\mathrm{D}}$ is the Dirac distribution. The coupling functions $\alpha$ and $\beta$ are given by

$\alpha\left(\boldsymbol{k}_{1}, \boldsymbol{k}_{2}\right)=\frac{\left(\boldsymbol{k}_{1}+\boldsymbol{k}_{2}\right) \cdot \boldsymbol{k}_{1}}{k_{1}^{2}}, \beta\left(\boldsymbol{k}_{1}, \boldsymbol{k}_{2}\right)=\frac{\left|\boldsymbol{k}_{1}+\boldsymbol{k}_{2}\right|^{2}\left(\boldsymbol{k}_{1} \cdot \boldsymbol{k}_{2}\right)}{2 k_{1}^{2} k_{2}^{2}}$,

and we defined the Fourier transforms as

$\delta(\boldsymbol{k})=\int \frac{\mathrm{d} \boldsymbol{x}}{(2 \pi)^{3}} \mathrm{e}^{-\mathrm{i} \boldsymbol{k} \cdot \boldsymbol{x}} \delta(\boldsymbol{x})$.

As in Crocce \& Scoccimarro (2006a,b), let us define the twocomponent vector $\psi$ as

$\psi(\boldsymbol{k}, \eta)=\left(\begin{array}{c}\psi_{1}(\boldsymbol{k}, \eta) \\ \psi_{2}(\boldsymbol{k}, \eta)\end{array}\right)=\left(\begin{array}{c}\delta(\boldsymbol{k}, \eta) \\ -\theta(\boldsymbol{k}, \eta) / f \mathcal{H}\end{array}\right)$,

where we have introduced the time coordinate $\eta$ defined from the linear growing rate $D_{+}$of the density contrast (normalized to unity today):

$\eta=\ln D_{+}(\tau) \quad$ with $\quad D_{+}(z=0)=1$.

Then, the equations of motion (11)-(12) can be written as

$\mathcal{O}\left(x, x^{\prime}\right) \cdot \psi\left(x^{\prime}\right)=K_{\mathrm{s}}\left(x ; x_{1}, x_{2}\right) \cdot \psi\left(x_{1}\right) \psi\left(x_{2}\right)$,

where we have introduced the coordinate $x=(\boldsymbol{k}, \eta, i)$ where $i=1,2$ is the discrete index of the two-component vectors. In Eq. (17) and in the following, we use the convention that repeated coordinates are integrated over as

$O\left(x, x^{\prime}\right) \cdot \psi\left(x^{\prime}\right)=\int \mathrm{d} \boldsymbol{k}^{\prime} \mathrm{d} \eta^{\prime} \sum_{i^{\prime}=1}^{2} O_{i, i^{\prime}}\left(\boldsymbol{k}, \eta ; \boldsymbol{k}^{\prime}, \eta^{\prime}\right) \psi_{i^{\prime}}\left(\boldsymbol{k}^{\prime}, \eta^{\prime}\right)$.

The matrix $O$ reads

$O\left(x, x^{\prime}\right)=\left(\begin{array}{cc}\frac{\partial}{\partial \eta} & -1 \\ 0 & \frac{\partial}{\partial \eta}-1\end{array}\right) \delta_{\mathrm{D}}\left(\boldsymbol{k}-\boldsymbol{k}^{\prime}\right), \delta_{\mathrm{D}}\left(\eta-\eta^{\prime}\right)$ whereas the symmetric vertex $K_{\mathrm{s}}\left(x ; x_{1}, x_{2}\right)=K_{\mathrm{s}}\left(x ; x_{2}, x_{1}\right)$ writes as

$$
\begin{aligned}
K_{\mathrm{s}}\left(x ; x_{1}, x_{2}\right)= & \delta_{\mathrm{D}}\left(\boldsymbol{k}_{1}+\boldsymbol{k}_{2}-\boldsymbol{k}\right) \delta_{\mathrm{D}}\left(\eta_{1}-\eta\right) \delta_{\mathrm{D}}\left(\eta_{2}-\eta\right) \\
& \times \gamma_{i ; i_{1}, i_{2}}^{s}\left(\boldsymbol{k}_{1}, \boldsymbol{k}_{2}\right)
\end{aligned}
$$

with

$\gamma_{1 ; 1,2}^{s}\left(\boldsymbol{k}_{1}, \boldsymbol{k}_{2}\right)=\frac{\alpha\left(\boldsymbol{k}_{2}, \boldsymbol{k}_{1}\right)}{2}, \quad \gamma_{1 ; 2,1}^{s}\left(\boldsymbol{k}_{1}, \boldsymbol{k}_{2}\right)=\frac{\alpha\left(\boldsymbol{k}_{1}, \boldsymbol{k}_{2}\right)}{2}$,

$\gamma_{2 ; 2,2}^{s}\left(\boldsymbol{k}_{1}, \boldsymbol{k}_{2}\right)=\beta\left(\boldsymbol{k}_{1}, \boldsymbol{k}_{2}\right)$,

and zero otherwise (Crocce \& Scoccimarro 2006a). We can note that all the dependence on cosmology is contained in the timeredshift relation $\eta \leftrightarrow z$. Indeed, the equation of motion (17) written in terms of the coordinate $\eta$ no longer involves timedependent factors such as $\Omega_{\mathrm{m}} / f^{2}$. Therefore, the evolution of the density field only depends on cosmology through the timecoordinate $\eta(z)$. In this article we study the system defined by the the equation of motion (17), which shows the exact solution (10). This will allow us to compare various expansion methods with exact nonlinear results.

\subsection{Linear regime}

On large scales or at early times where the density and velocity fluctuations are small, one can linearize the equation of motion (17), which yields $O \cdot \psi_{\mathrm{L}}=0$. This gives the two linear modes:

$\psi_{+}=\mathrm{e}^{\eta}\left(\begin{array}{l}1 \\ 1\end{array}\right), \quad \psi_{-}=\left(\begin{array}{l}1 \\ 0\end{array}\right)$

Of course we recover the linear growing mode $\psi_{+}$of the gravitational dynamics, since approximation (5) is valid in this case. However, the usual decaying mode $\psi_{-}$has been changed to a constant mode. As seen in Eq. (23), it corresponds to a mere perturbation of the density field that is transported by the unchanged velocity field. Indeed, since the velocity field is now decoupled from the density field, it obeys a first-order differential equation in the linear regime (rather than a second-order differential equation), which only admits one linear mode. As usual we define the initial conditions by the linear growing mode $\psi_{\mathrm{L}}$ :

$\psi_{\mathrm{L}}(x)=\mathrm{e}^{\eta} \delta_{\mathrm{L} 0}(\boldsymbol{k})\left(\begin{array}{l}1 \\ 1\end{array}\right)$

where $\delta_{\mathrm{L} 0}(\boldsymbol{k})$ is the linear density contrast today at redshift $z=0$. In this fashion the system (11)-(12) that we study here agrees with the gravitational dynamics in the linear regime. Besides, from Eqs. (5) and (10), we see that the displacement field $s_{\mathrm{L} 0}(\boldsymbol{q})$ obeys

$\nabla_{q} \cdot s_{\mathrm{L} 0}=-\delta_{\mathrm{L} 0}$.

Moreover, we assume Gaussian homogeneous and isotropic initial conditions defined by the linear power spectrum $P_{\mathrm{L} 0}(k)$ :

$\left\langle\delta_{\mathrm{L} 0}\left(\boldsymbol{k}_{1}\right) \delta_{\mathrm{L} 0}\left(\boldsymbol{k}_{2}\right)\right\rangle=\delta_{\mathrm{D}}\left(\boldsymbol{k}_{1}+\boldsymbol{k}_{2}\right) P_{\mathrm{L} 0}\left(k_{1}\right)$.

Then, as for the gravitational dynamics studied in Valageas (2007), the linear two-point correlation function $G_{\mathrm{L}}\left(x_{1}, x_{2}\right)$ reads as:

$\begin{aligned} G_{\mathrm{L}}\left(x_{1}, x_{2}\right) & =\left\langle\psi_{\mathrm{L}}\left(x_{1}\right) \psi_{\mathrm{L}}\left(x_{2}\right)\right\rangle \\ & =\delta_{\mathrm{D}}\left(\boldsymbol{k}_{1}+\boldsymbol{k}_{2}\right) \mathrm{e}^{\eta_{1}+\eta_{2}} P_{\mathrm{L} 0}\left(k_{1}\right)\left(\begin{array}{ll}1 & 1 \\ 1 & 1\end{array}\right) .\end{aligned}$ 
As in Valageas (2004), it is convenient to introduce the response function $R\left(x_{1}, x_{2}\right)$ (related to the propagator used in Crocce \& Scoccimarro 2006a,b, see Sect. 3.2.2 below) defined by the functional derivative

$R\left(x_{1}, x_{2}\right)=\left\langle\frac{\delta \psi\left(x_{1}\right)}{\delta \zeta\left(x_{2}\right)}\right\rangle_{\zeta=0}$,

where $\zeta(x)$ is a "noise" added to the r.h.s. in Eq. (17). Thus, $R\left(x_{1}, x_{2}\right)$ measures the linear response of the system to an external source of noise. Because of causality it contains an Heaviside factor $\theta\left(\eta_{1}-\eta_{2}\right)$ since the field $\psi\left(x_{1}\right)$ can only depend on the values of the "noise" at earlier times $\eta_{2} \leq \eta_{1}$. Moreover, it satisfies the initial condition:

$\eta_{1} \rightarrow \eta_{2}^{+}: R\left(x_{1}, x_{2}\right) \rightarrow \delta_{\mathrm{D}}\left(\boldsymbol{k}_{1}-\boldsymbol{k}_{2}\right) \delta_{i_{1}, i_{2}}$.

In the linear regime, the response $R_{\mathrm{L}}$ can be obtained from the initial condition (29) and the linear dynamics $O \cdot R_{\mathrm{L}}=0$ for $\eta_{1}>\eta_{2}$ (as implied by the definition (28) and $O \cdot \psi_{\mathrm{L}}=0$ ). This yields (Crocce et al. 2006)

$$
\begin{aligned}
R_{\mathrm{L}}\left(x_{1}, x_{2}\right)= & \delta_{\mathrm{D}}\left(\boldsymbol{k}_{1}-\boldsymbol{k}_{2}\right) \theta\left(\eta_{1}-\eta_{2}\right) \\
& \times\left\{\mathrm{e}^{\eta_{1}-\eta_{2}}\left(\begin{array}{ll}
0 & 1 \\
0 & 1
\end{array}\right)+\left(\begin{array}{cc}
1 & -1 \\
0 & 0
\end{array}\right)\right\} .
\end{aligned}
$$

This expression holds for any cosmology, whereas for the case of the gravitational dynamics factors, such as $\Omega_{\mathrm{m}} / f^{2}$, lead to a small explicit dependence on cosmological parameters. Note that by symmetry the two-point correlation $G$ has the form

$G\left(x_{1}, x_{2}\right)=\delta_{\mathrm{D}}\left(\boldsymbol{k}_{1}+\boldsymbol{k}_{2}\right) G_{i_{1}, i_{2}}\left(k_{1} ; \eta_{1}, \eta_{2}\right)$

with

$G_{i_{1}, i_{2}}\left(k ; \eta_{1}, \eta_{2}\right)=G_{i_{2}, i_{1}}\left(k ; \eta_{2}, \eta_{1}\right)$

whereas the response function has the form

$R\left(x_{1}, x_{2}\right)=\delta_{\mathrm{D}}\left(\boldsymbol{k}_{1}-\boldsymbol{k}_{2}\right) \theta\left(\eta_{1}-\eta_{2}\right) R_{i_{1}, i_{2}}\left(k_{1} ; \eta_{1}, \eta_{2}\right)$.

On the other hand, as noticed above, the linear two-point functions obey

$O(x, z) \cdot G_{\mathrm{L}}(z, y)=0, \quad O(x, z) \cdot R_{\mathrm{L}}(z, y)=\delta_{\mathrm{D}}(x-y)$.

This can also be checked from the explicit expressions (27), (30). Finally, it is convenient to define the power per logarithmic wavenumber $\Delta^{2}(k)$ by

$\Delta^{2}(k)=4 \pi k^{3} P(k), \quad \Delta^{2}\left(k ; \eta_{1}, \eta_{2}\right)=4 \pi k^{3} G_{11}\left(k ; \eta_{1}, \eta_{2}\right)$

where the second expression generalizes $\Delta^{2}(k)$ at different times. Note that for $\eta_{1} \neq \eta_{2}$ we can have $\Delta^{2}\left(k ; \eta_{1}, \eta_{2}\right)<0$, whereas at equal times we have $\Delta^{2}(k ; \eta, \eta) \geq 0$. Then we have, for instance,

$\left\langle\delta\left(\boldsymbol{x}_{1}\right) \delta\left(\boldsymbol{x}_{2}\right)\right\rangle=\int_{0}^{\infty} \frac{\mathrm{d} k}{k} \Delta^{2}(k) \frac{\sin k\left|\boldsymbol{x}_{1}-\boldsymbol{x}_{2}\right|}{k\left|\boldsymbol{x}_{1}-\boldsymbol{x}_{2}\right|}$.

Thus, for a CDM cosmology the linear power $\Delta_{\mathrm{L} 0}^{2}(k)$ grows as $k^{4}$ at low $k$ and as $\ln k$ at high $k$.

\section{Path-integral formalism}

\subsection{Differential form}

As in Valageas $(2004,2007)$ we can apply a path-integral approach to the hydrodynamical system described in Sect. 2.1. Let us briefly recall how this can be done from the differential Eq. (17) (see also Martin et al. 1973; Phythian 1977). In order to explicitly include the initial conditions, we rewrite Eq. (17) as

$O \cdot \psi=K_{\mathrm{s}} \cdot \psi \psi+\mu_{\mathrm{I}}$

with $\psi=0$ for $\eta<\eta_{\text {I }}$ and

$\mu_{\mathrm{I}}(x)=\delta_{\mathrm{D}}\left(\eta-\eta_{\mathrm{I}}\right) \mathrm{e}^{\eta_{\mathrm{I}}} \delta_{\mathrm{L} 0}(\boldsymbol{k})\left(\begin{array}{l}1 \\ 1\end{array}\right)=\delta_{\mathrm{D}}\left(\eta-\eta_{\mathrm{I}}\right) \psi_{\mathrm{I}}(\bar{x})$,

where we have introduced the coordinate $\bar{x}$ :

$\bar{x}=(\boldsymbol{k}, i)$ and $\psi_{\mathrm{I}}(\bar{x})=\psi_{\mathrm{L}}\left(\bar{x}, \eta_{\mathrm{I}}\right)$.

Thus, the source $\mu_{\mathrm{I}}$ (which formally plays the role of some external noise) merely provides the initial conditions at time $\eta_{\mathrm{I}}$, obtained from the linear growing mode (24). We shall eventually take the limit $\eta_{\mathrm{I}} \rightarrow-\infty$. Next, we define the generating functional $Z[j]$ by

$Z[j]=\left\langle\mathrm{e}^{j \cdot \psi}\right\rangle=\int\left[\mathrm{d} \mu_{\mathrm{I}}\right] \mathrm{e}^{j \cdot \psi\left[\mu_{\mathrm{I}}\right]-\frac{1}{2} \mu_{\mathrm{I}} \cdot \Delta_{\mathrm{I}}^{-1} \cdot \mu_{\mathrm{I}}}$,

where we took the average over the Gaussian initial conditions

$\left\langle\mu_{\mathrm{I}}\right\rangle=0, \quad\left\langle\mu_{\mathrm{I}}\left(x_{1}\right) \mu_{\mathrm{I}}\left(x_{2}\right)\right\rangle=\Delta_{\mathrm{I}}\left(x_{1}, x_{2}\right)$,

with

$\Delta_{\mathrm{I}}\left(x_{1}, x_{2}\right)=\delta_{\mathrm{D}}\left(\eta_{1}-\eta_{\mathrm{I}}\right) \delta_{\mathrm{D}}\left(\eta_{2}-\eta_{\mathrm{I}}\right) G_{\mathrm{I}}\left(\bar{x}_{1}, \bar{x}_{2}\right)$
$G_{\mathrm{I}}\left(\bar{x}_{1}, \bar{x}_{2}\right)=G_{\mathrm{L}}\left(\bar{x}_{1}, \eta_{\mathrm{I}} ; \bar{x}_{2}, \eta_{\mathrm{I}}\right)$

All statistical properties of the field $\psi$ may be obtained from $Z[j]$. It is convenient to write Eq. (40) as

$$
\begin{aligned}
Z[j]= & \int\left[\mathrm{d} \mu_{\mathrm{I}}\right][\mathrm{d} \psi]|\operatorname{det} M| \delta_{\mathrm{D}}\left(\mu_{\mathrm{I}}-O \cdot \psi+K_{\mathrm{S}} \cdot \psi \psi\right) \\
& \times \mathrm{e}^{j \cdot \psi-\frac{1}{2} \mu_{\mathrm{I}} \cdot \Delta_{\mathrm{I}}^{-1} \cdot \mu_{\mathrm{I}}}
\end{aligned}
$$

where the Jacobian $|\operatorname{det} M|$ is defined by the functional derivative $M=\delta \mu_{\mathrm{I}} / \delta \psi$. As in Valageas (2007), a simple computation shows that this Jacobian is equal to an irrelevant constant. Then, introducing an imaginary ghost field $\lambda$ to express the Dirac as an exponential and performing the Gaussian integration over $\mu_{\mathrm{I}}$, we obtain

$Z[j]=\int[\mathrm{d} \psi][\mathrm{d} \lambda] \mathrm{e}^{j \cdot \psi+\lambda \cdot\left(-O \cdot \psi+K_{\mathrm{s}} \cdot \psi \psi\right)+\frac{1}{2} \lambda \cdot \Delta_{\mathrm{I}} \cdot \lambda}$.

Thus, the statistical properties of the system (17) are described by the action $S[\psi, \lambda]$ defined by

$S[\psi, \lambda]=\lambda \cdot\left(O \cdot \psi-K_{\mathrm{S}} \cdot \psi \psi\right)-\frac{1}{2} \lambda \cdot \Delta_{\mathrm{I}} \cdot \lambda$.

Moreover, we can note that adding a "noise" $\zeta$ to the r.h.s. of Eq. (17) amounts to changing $\mu_{\mathrm{I}} \rightarrow \mu_{\mathrm{I}}+\zeta$, which translates into $S \rightarrow S-\lambda \cdot \zeta$. Therefore, functional derivatives with respect to $\zeta$ are equivalent to insertions of the ghost field $\lambda$. In particular, we have

$R\left(x_{1}, x_{2}\right)=\left\langle\psi\left(x_{1}\right) \lambda\left(x_{2}\right)\right\rangle, \quad\langle\lambda\rangle=0, \quad\langle\lambda \lambda\rangle=0$. 
The response function $R$ is also related to the correlation with the initial conditions $\mu_{\mathrm{I}}$ through

$$
\begin{aligned}
\left\langle\psi \mu_{\mathrm{I}}\right\rangle & =\left\langle\psi\left(O \cdot \psi-K_{\mathrm{s}} \cdot \psi \psi\right)\right\rangle \\
& =\int[\mathrm{d} \psi][\mathrm{d} \lambda] \psi\left[-\frac{\delta}{\delta \lambda}+\Delta_{\mathrm{I}} \cdot \lambda\right] \mathrm{e}^{\lambda \cdot\left(-O \cdot \psi+K_{\mathrm{s}} \cdot \psi \psi\right)+\frac{1}{2} \lambda \cdot \Delta_{\mathrm{I}} \cdot \lambda} \\
& =\left\langle\psi\left(\Delta_{\mathrm{I}} \cdot \lambda\right)\right\rangle=R \cdot \Delta_{\mathrm{I}}
\end{aligned}
$$

since the integral of a total derivative vanishes, and we have used the symmetry of $\Delta_{\mathrm{I}}$. This also reads as

$\left\langle\psi\left(x_{1}\right) \psi_{\mathrm{I}}\left(\bar{x}_{2}\right)\right\rangle=R\left(x_{1} ; \bar{x}, \eta_{\mathrm{I}}\right) \times G_{\mathrm{I}}\left(\bar{x} ; \bar{x}_{2}\right)$,

where we define the cross-product $\times$ as the dot product (18) without integration over time, such as:

$R \times \psi_{\mathrm{I}}=\int \mathrm{d} \boldsymbol{k}^{\prime} \sum_{j=1}^{2} R_{i j}\left(\boldsymbol{k}, \eta ; \boldsymbol{k}^{\prime}, \eta_{\mathrm{I}}\right) \psi_{\mathrm{I} j}\left(\boldsymbol{k}^{\prime}\right)$.

\subsection{Integral form}

In order to make the connection with the approach developed in Crocce \& Scoccimarro (2006a,b), we describe here how the same path-integral method can be applied to the equation of motion (17) written in integral form.

\subsubsection{Letting $\eta_{1} \rightarrow-\infty$}

First, as in Valageas (2001) (see also Scoccimarro 2000), we can integrate the equation of motion (17) as

$\psi(x)=\psi_{\mathrm{L}}(x)+\tilde{K}_{\mathrm{S}}\left(x ; x_{1}, x_{2}\right) \cdot \psi\left(x_{1}\right) \psi\left(x_{2}\right)$

with

$O \cdot \tilde{K}_{\mathrm{S}}=K_{\mathrm{s}}$ or $\quad \tilde{K}_{\mathrm{S}}=R_{\mathrm{L}} \cdot K_{\mathrm{S}}$

as seen from Eq. (34). Here the initial time $\eta_{\mathrm{I}}$ no longer appears, because we have already taken the limit $\eta_{\mathrm{I}} \rightarrow-\infty$. Then, following the same procedure as in Sect. 3.1 we can write

$Z[j]=\left\langle\mathrm{e}^{j \cdot \psi}\right\rangle=\int\left[\mathrm{d} \psi_{\mathrm{L}}\right] \mathrm{e}^{j \cdot \psi\left[\psi_{\mathrm{L}}\right]-\frac{1}{2} \psi_{\mathrm{L}} \cdot G_{\mathrm{L}}^{-1} \cdot \psi_{\mathrm{L}}}$

Introducing again an imaginary field $\chi$ to impose the constraint associated with the equation of motion (51), we finally obtain (the Jacobian is equal to unity):

$Z[j]=\int[\mathrm{d} \psi][\mathrm{d} \chi] \mathrm{e}^{j \cdot \psi+\chi \cdot\left(-\psi+\tilde{K}_{\mathrm{s}} \cdot \psi \psi\right)+\frac{1}{2} \chi \cdot G_{\mathrm{L}} \cdot \chi}$.

Thus, the statistical properties of the system (51) are now described by the action $\mathcal{S}[\psi, \chi]$ defined by

$\mathcal{S}[\psi, \chi]=\chi \cdot\left(\psi-\tilde{K}_{\mathrm{s}} \cdot \psi \psi\right)-\frac{1}{2} \chi \cdot G_{\mathrm{L}} \cdot \chi$.

Note that this formulation is equivalent to the one described in Sect. 3.1 except that we have already taken the limit $\eta_{\mathrm{I}} \rightarrow-\infty$ directly into the equation of motion (51). From the response field $\chi$, we can again obtain a new response function $\mathcal{R}$ associated with Eq. (51). From the comparison with Eq. (45), we see that we have the relations between both approaches:

$\chi=\lambda \cdot O, \quad \mathcal{R}=\langle\psi \chi\rangle=R \cdot O=\left\langle\frac{\delta \psi}{\delta \psi_{\mathrm{L}}}\right\rangle$, where in the last expression we recall that from Eq. (51) a variation with respect to an external noise $\zeta$ can be seen as a variation with respect to $\psi_{\mathrm{L}}$. In the linear regime we simply have $\mathcal{R}_{\mathrm{L}}(x, y)=\delta_{\mathrm{D}}(x-y)$. Moreover, in a fashion similar to Eq. (49) we have the property

$$
\begin{aligned}
\left\langle\psi \psi_{\mathrm{L}}\right\rangle & =\left\langle\psi\left(\psi-\tilde{K}_{\mathrm{s}} \cdot \psi \psi\right)\right\rangle \\
& =\int[\mathrm{d} \psi][\mathrm{d} \chi] \psi\left[-\frac{\delta}{\delta \chi}+G_{\mathrm{L}} \cdot \chi\right] \mathrm{e}^{\chi \cdot\left(-\psi+\tilde{K}_{\mathrm{s}} \cdot \psi \psi\right)+\frac{1}{2} \chi \cdot G_{\mathrm{L}} \cdot \chi} \\
& =\left\langle\psi\left(G_{\mathrm{L}} \cdot \chi\right)\right\rangle
\end{aligned}
$$

which yields the relation

$\left\langle\psi\left(x_{1}\right) \psi_{\mathrm{L}}\left(x_{2}\right)\right\rangle=\mathcal{R}\left(x_{1}, x\right) \cdot G_{\mathrm{L}}\left(x, x_{2}\right)$,

where we use the symmetry of $G_{\mathrm{L}}$.

\subsubsection{Integral form with finite $\eta_{\text {। }}$}

Finally, as in Crocce \& Scoccimarro (2006a,b), it is possible to apply the initial conditions at some finite time $\eta_{\mathrm{I}}$, as in Sect. 3.1. Thus, we may write the linear growing mode $\psi_{\mathrm{L}}$ at times $\eta>$ $\eta_{\mathrm{I}}$ as

$\psi_{\mathrm{L}}(x)=R_{\mathrm{L}}\left(x ; \bar{x}^{\prime}, \eta_{\mathrm{I}}\right) \times \psi_{\mathrm{I}}\left(\bar{x}^{\prime}\right)$

where $\psi_{\mathrm{I}}$ was defined in Eq. (39) and the cross-product $X$ in Eq. (50). Then, following the same procedure as in Eqs. (51)-(54), where the Gaussian average is now taken over the field $\psi_{\mathrm{I}}$ with two-point correlation $G_{\mathrm{I}}$, we now obtain the generating functional:

$$
\begin{aligned}
Z[j] & =\int\left[\mathrm{d} \psi_{\mathrm{I}}\right][\mathrm{d} \psi][\mathrm{d} \chi] \mathrm{e}^{j \cdot \psi+\chi \cdot\left(R_{\mathrm{L}} \times \psi_{\mathrm{I}}-\psi+\tilde{K}_{\mathrm{S}} \cdot \psi \psi\right)-\frac{1}{2} \psi_{\mathrm{I}} \times G_{\mathrm{I}}^{-1} \times \psi_{\mathrm{I}}} \\
& =\int[\mathrm{d} \psi][\mathrm{d} \chi] \mathrm{e}^{j \cdot \psi+\chi \cdot\left(-\psi+\tilde{K}_{\mathrm{S}} \cdot \psi \psi\right)+\frac{1}{2} \chi \cdot\left(R_{\mathrm{L}} \times G_{\mathrm{I}} \times R_{\mathrm{L}}^{\mathrm{T}}\right) \cdot \chi}
\end{aligned}
$$

Of course, we can check that, by taking the limit $\eta_{\mathrm{I}} \rightarrow-\infty$ in Eq. (60), we recover Eq. (54) since we have

$\eta_{1}, \eta_{2}>\eta_{\mathrm{I}}: G_{\mathrm{L}}\left(\eta_{1}, \eta_{2}\right)=R_{\mathrm{L}}\left(\eta_{1}, \eta_{\mathrm{I}}\right) \times G_{\mathrm{I}} \times R_{\mathrm{L}}^{\mathrm{T}}\left(\eta_{2}, \eta_{\mathrm{I}}\right)$.

The system is now described by the action $\tilde{\mathcal{S}}[\psi, \chi]$ defined by

$\tilde{\mathcal{S}}[\psi, \chi]=\chi \cdot\left(\psi-\tilde{K}_{\mathrm{s}} \cdot \psi \psi\right)-\frac{1}{2} \chi \cdot\left(R_{\mathrm{L}} \times G_{\mathrm{I}} \times R_{\mathrm{L}}^{\mathrm{T}}\right) \cdot \chi$.

Next, we can define a response function with respect to the initial conditions by

$\tilde{\mathcal{R}}\left(x_{1}, \bar{x}_{2}\right)=\left\langle\frac{\delta \psi\left(x_{1}\right)}{\delta \psi_{\mathrm{I}}\left(\bar{x}_{2}\right)}\right\rangle=\left\langle\psi\left(x_{1}\right) \chi(x) \cdot R_{\mathrm{L}}\left(x ; \bar{x}_{2}, \eta_{\mathrm{I}}\right)\right\rangle$.

From the comparison of (62) with (46), we obtain $\chi=\lambda \cdot O$ and $\tilde{\mathcal{R}}\left(x_{1}, \bar{x}_{2}\right)=R \cdot O \cdot R_{\mathrm{L}}=R\left(x_{1} ; \bar{x}_{2}, \eta_{\mathrm{I}}\right)$.

Thus, the response $\tilde{\mathcal{R}}\left(x_{1}, \bar{x}_{2}\right)$, which is called the "propagator $G_{i_{1} i_{2}}\left(k_{1}, \eta_{1}\right) \delta_{\mathrm{D}}\left(\boldsymbol{k}_{1}-\boldsymbol{k}_{2}\right)$ " in Crocce \& Scoccimarro $(2006 \mathrm{a}, \mathrm{b})$ is equal to the response function $R$ of Sect. 3.1 restricted to time $\eta_{2}=\eta_{\mathrm{I}}$, without taking the limit $\eta_{\mathrm{I}} \rightarrow-\infty$. Finally, we can note that from Eq. (49) we have

$\left\langle\psi\left(x_{1}\right) \psi_{\mathrm{I}}\left(\bar{x}_{2}\right)\right\rangle=\tilde{\mathcal{R}}\left(x_{1} ; \bar{x}\right) \times G_{\mathrm{I}}\left(\bar{x}, \bar{x}_{2}\right)$.

This relation was obtained in Crocce \& Scoccimarro (2006b) from a diagrammatic approach. Thus, we see that the three approaches (46), (55), and (62) are closely related. In the integral 
method we simply absorb the matrix $O$ into the response field $\chi$. Next, we can either take the limit $\eta_{\mathrm{I}} \rightarrow-\infty$ from the start, as for the action $\mathcal{S}$, or keep a finite $\eta_{\mathrm{I}}$ in the computation, as for $\tilde{\mathcal{S}}$. Then, we can take $\eta_{\mathrm{I}} \rightarrow-\infty$ in the final results for the nonlinear two-point correlation. Note, however, that for the approach of Crocce \& Scoccimarro (2006a,b), which corresponds to the action $\tilde{\mathcal{S}}$, it is not possible to take this limit in a practical manner, since one needs to keep track of the response $\tilde{\mathcal{R}}$, which has no finite limit for $\eta_{\mathrm{I}} \rightarrow-\infty$. This leads to somewhat more complicated expressions than for the approaches based on the actions $S$ and $S$ of Eqs. (46), (55), where the response functions $R$ and $\mathcal{R}$ remain well-defined for $\eta_{\mathrm{I}} \rightarrow-\infty$. Of course, the analysis described above also applies to the case of the gravitational dynamics.

\section{Large- $N$ expansions}

The path integrals (45), (54), and (60) can be computed by expanding over powers of the non-Gaussian part (i.e. over powers of $K_{\mathrm{S}}$ or $\tilde{K}_{\mathrm{s}}$ ). This actually gives the usual perturbative expansion over powers of the linear power spectrum $P_{\mathrm{L}}$ (see also Valageas 2001, 2004, for the case of the Vlasov equation of motion). On the other hand, these path integrals may also be studied through large- $N$ expansions as in Valageas (2004). We focus below on the differential form (45), but the formalism also applies to the integral forms (54) and (60). Thus, one considers the generating functional $Z_{N}[j, h]$ defined by

$Z_{N}[j, h]=\int[\mathrm{d} \psi][\mathrm{d} \lambda] \mathrm{e}^{N[j \cdot \psi+h \cdot \lambda-S[\psi, \lambda]]}$,

and one looks for an expansion over powers of $1 / N$, taking eventually $N=1$ into the results. As discussed in Valageas (2004), the large- $N$ expansions may also be derived from a generalization of the dynamics to $N$ fields $\psi^{(\alpha)}$. This yields the same results once we deal with the long-wavelength divergences that constrain which subsets of diagrams need to be gathered.

The interest of such large- $N$ expansions is to provide new systematic expansion schemes that may show improved convergence properties as compared with the standard perturbation theory. Besides, it is clear from Eq. (66) that the symmetries of the system (e.g. invariance through translations) are automatically conserved at any order. These methods have been applied to many fields of theoretical physics, such as quantum field theory (e.g. Zinn-Justin 1989; Berges 2002), statistical physics (e.g. study of growing interfaces described by the Kardar-ParisiZhang equation, Doherty et al. 1994), and turbulence (e.g. Mou $\&$ Weichman 1993). They are closely related at lowest order to the so-called "mode-coupling approximations" used for critical dynamics, liquids, or glassy systems (Bouchaud et al. 1996), and to the "direct interaction approximation" used for turbulent flows (Kraichnan 1961). Therefore, it is natural to investigate their application to the cosmological gravitational dynamics described by Eqs. (1)-(3), which are similar to the Navier-Stokes equations. In some cases (e.g. Berges 2002), it has been found that, whereas the simplest perturbative expansions give rise to secular terms (which grow as powers of time), the 2PI effective action method derived from such a large- $N$ method (discussed below in Sect. 4.2) could achieve a non-secular behavior and display relaxation processes. Of course, the actual behavior of such schemes depends on the specific problem. It has already been shown in Valageas (2007) that, for the case of the gravitational dynamics in the expanding Universe, the large- $N$ expansions indeed show a qualitative improvement over standard perturbation theory at one-loop order, as they display bounded oscillations (for the steepest-descent method) or decaying oscillations (for the 2PI effective action method) for the response functions instead of the secular terms encountered in the standard perturbative expansion (which gives increasingly large powers of time $D(\tau)^{p}$ at higher orders). In this article, we investigate whether this good behavior extends to higher orders in the case of the Zeldovich dynamics.

We discuss below both "linear schemes", such as the standard perturbation theory or the steepest-descent method of Sect. 4.1, which involve expansions over linear two-point functions, and "nonlinear schemes", such as the 2PI effective action method of Sect. 4.2, which involve expansions over nonlinear two-point functions themselves. By expanding different intermediate quantities or different equations (derived from the same equation of motion), one obtains different methods that also correspond to different partial resummations.

\subsection{Steepest-descent method}

A first approach to handle the large- $N$ limit of Eq. (66) is to use a steepest-descent method (also called a semi-classical or loopwise expansion in the case of usual quantum field theory with $\hbar=1 / N)$. For auxiliary correlation and response functions $G_{0}$ and $R_{0}$, this yields the equations (Valageas 2004)

$O(x, z) \cdot G_{0}(z, y)=0$

$O(x, z) \cdot R_{0}(z, y)=\delta_{\mathrm{D}}(x-y)$

$R_{0}(x, z) \cdot O(z, y)=\delta_{\mathrm{D}}(x-y)$,

whereas the actual correlation and response functions obey

$O(x, z) \cdot G(z, y)=\Sigma(x, z) \cdot G(z, y)+\Pi(x, z) \cdot R^{\mathrm{T}}(z, y)$

$O(x, z) \cdot R(z, y)=\delta_{\mathrm{D}}(x-y)+\Sigma(x, z) \cdot R(z, y)$

$R(x, z) \cdot O(z, y)=\delta_{\mathrm{D}}(x-y)+R(x, z) \cdot \Sigma(z, y)$

where the self-energy terms $\Sigma$ and $\Pi$ are given at one-loop order by

$\Sigma(x, y)=4 K_{\mathrm{s}}\left(x ; x_{1}, x_{2}\right) K_{\mathrm{s}}\left(z ; y, z_{2}\right) R_{0}\left(x_{1}, z\right) G_{0}\left(x_{2}, z_{2}\right)$

$\Pi(x, y)=2 K_{\mathrm{s}}\left(x ; x_{1}, x_{2}\right) K_{\mathrm{s}}\left(y ; y_{1}, y_{2}\right) G_{0}\left(x_{1}, y_{1}\right) G_{0}\left(x_{2}, y_{2}\right)$.

Note that Eqs. (67)-(72) are exact and that the expansion over powers of $1 / N$ only enters the expression of the self-energy (73), (74). Here we only kept the lowest-order terms (see Valageas 2004 for the next-order terms). We also took the limit $\eta_{\mathrm{I}} \rightarrow$ $-\infty$ so that terms involving $\Delta_{\mathrm{I}}$ vanish. The comparison of Eqs. (67), (68) with Eq. (34) shows that the auxiliary matrices $G_{0}$ and $R_{0}$ are actually equal to their linear counterparts:

$G_{0}=G_{\mathrm{L}}, \quad R_{0}=R_{\mathrm{L}}$.

Next, substituting $G_{0}$ and $R_{0}$ into Eqs. (73), (74), we obtain the self-energies at one-loop order. First, we can note that $\Sigma$ has the same form (33) as the response $R$, whereas $\Pi$ is symmetric and has the same form (31) as the two-point correlation $G$. Then, a simple calculation gives

$$
\begin{aligned}
\Sigma_{0}\left(x_{1}, x_{2}\right)= & -\omega_{1}^{2} \theta\left(\eta_{1}-\eta_{2}\right) \delta_{\mathrm{D}}\left(\boldsymbol{k}_{1}-\boldsymbol{k}_{2}\right) \\
& \times\left[\mathrm{e}^{2 \eta_{1}}\left(\begin{array}{ll}
0 & 1 \\
0 & 1
\end{array}\right)+\mathrm{e}^{\eta_{1}+\eta_{2}}\left(\begin{array}{cc}
1 & -1 \\
0 & 0
\end{array}\right)\right],
\end{aligned}
$$

where we define $\omega_{1}=\omega\left(k_{1}\right)$ as

$\omega(k)=k \sigma_{v}$ with $\sigma_{v}^{2}=\frac{1}{3}\left\langle s_{\mathrm{L} 0}^{2}\right\rangle=\frac{4 \pi}{3} \int_{0}^{\infty} \mathrm{d} w P_{\mathrm{L} 0}(w)$. 
Here $\sigma_{v}^{2}$ is the variance of the one-dimensional displacement field $s_{\mathrm{L} 0}$ (or of the one-dimensional velocity dispersion up to a normalization factor). On the other hand, $\Pi$ is given at one-loop order by

$\Pi_{0}\left(x_{1}, x_{2}\right)=\delta_{\mathrm{D}}\left(\boldsymbol{k}_{1}+\boldsymbol{k}_{2}\right) \mathrm{e}^{2 \eta_{1}+2 \eta_{2}} \Pi_{0}\left(k_{1}\right)$,

with

$$
\begin{aligned}
\Pi_{0}(k)= & 2 \int \mathrm{d} \boldsymbol{k}_{1} \mathrm{~d} \boldsymbol{k}_{2} \delta_{\mathrm{D}}\left(\boldsymbol{k}_{1}+\boldsymbol{k}_{2}-\boldsymbol{k}\right) P_{\mathrm{L} 0}\left(k_{1}\right) P_{\mathrm{L} 0}\left(k_{2}\right) \\
& \times\left(\begin{array}{cc}
\pi_{1}^{2} & \pi_{1} \pi_{2} \\
\pi_{1} \pi_{2} & \pi_{2}^{2}
\end{array}\right)
\end{aligned}
$$

and

$\pi_{1}=\frac{\alpha\left(\boldsymbol{k}_{1}, \boldsymbol{k}_{2}\right)+\alpha\left(\boldsymbol{k}_{2}, \boldsymbol{k}_{1}\right)}{2}, \quad \pi_{2}=\beta\left(\boldsymbol{k}_{1}, \boldsymbol{k}_{2}\right)$.

Then, the response $R$ and the correlation $G$ can be obtained by integrating Eqs. (70), (71).

\subsection{The $2 P I$ effective action method}

As described in Valageas (2004), a second approach is to first introduce the double Legendre transform $\Gamma[\psi, G]$ of the functional $W=\ln Z$ (with respect to both the field $\psi$ and its twopoint correlation $G$ ) and next to apply the $1 / N$ expansion to $\Gamma$. This "2PI effective action"method yields the same Eqs. (70)(72), and the self-energy shows the same structure at one-loop order as (73), (74) where $G_{0}$ and $R_{0}$ are replaced by $G$ and $R$ :

$\Sigma(x, y)=4 K_{\mathrm{s}}\left(x ; x_{1}, x_{2}\right) K_{\mathrm{s}}\left(z ; y, z_{2}\right) R\left(x_{1}, z\right) G\left(x_{2}, z_{2}\right)$

$\Pi(x, y)=2 K_{\mathrm{s}}\left(x ; x_{1}, x_{2}\right) K_{\mathrm{s}}\left(y ; y_{1}, y_{2}\right) G\left(x_{1}, y_{1}\right) G\left(x_{2}, y_{2}\right)$.

Thus, the direct steepest-descent method yields a series of linear equations that can be solved directly, whereas the 2PI effective action method gives a system of nonlinear equations (through the dependence on $G$ and $R$ of $\Sigma$ and $\Pi$ ) that usually must be solved numerically by an iterative scheme. However, thanks to the Heaviside factors appearing in the response $R$ and the selfenergy $\Sigma$, these equations can be solved directly by integrating forward over the time $\eta_{1}$.

\subsection{Role of self-energy terms}

From Eq. (71) we can see that the self-energy $\Sigma$ plays the role of a damping term. Indeed, Eq. (71) has the form $\partial R / \partial \eta_{1}=\Sigma \cdot R$ so that large negative values of $\Sigma$ are associated with a strong damping of the response function (Exact details are somewhat more intricate since Eq. (71) is actually an integro-differential equation.) This agrees with Eq. (76) which shows that the one-loop self-energy $\Sigma_{0}$ becomes large and negative at high $k$ as $\Sigma_{0} \propto-k^{2}$. Thus, the self-energy $\Sigma$ encodes the loss of memory associated with the nonlinear dynamics.

On the other hand, we can see from Eq. (70) that the selfenergy $\Pi$ is associated with the continuous production of power due to nonlinear mode couplings. Indeed, we can see from Eqs. (70), (72) that the correlation $G$ can also be written in terms of the response $R$ as

$G\left(x_{1}, x_{2}\right)=R \times G_{\mathrm{I}} \times R^{\mathrm{T}}+R \cdot \Pi \cdot R^{\mathrm{T}}$,

and we let $\eta_{\mathrm{I}} \rightarrow-\infty$. The physical meaning of Eq. (83) is clear. The first term on the right hand side means that the fluctuations at the initial time $\eta_{\mathrm{I}}$ are merely transported forward in time through the response $R$. This is the only nonzero term in the linear regime (with $R=R_{\mathrm{L}}$ hence $G=G_{\mathrm{L}}$ ). The effect of the nonlinear dynamics is to modify the transport matrix $R$ and to add a second term to the right hand side of Eq. (83). The latter has the meaning of a source term that produces fluctuations with two-point correlation $\Pi\left(\eta_{1}^{\prime}, \eta_{2}^{\prime}\right)$ at the times $\left(\eta_{1}^{\prime}, \eta_{2}^{\prime}\right)$ that are next transported forward to later times $\left(\eta_{1}, \eta_{2}\right)$ by the matrices $R\left(\eta_{1}, \eta_{1}^{\prime}\right)$ and $R^{\mathrm{T}}\left(\eta_{2}^{\prime}, \eta_{2}\right)$.

\section{Running with a high-k cutoff}

In a recent paper, Matarrese \& Pietroni (2007a) introduce another approach to studying the gravitational dynamics within the hydrodynamical framework. It is also based on a path-integral formulation. Although they use the integral form of the equations of motion, as in Sect. 3.2, we briefly describe in this section how this method may be applied to the path integral (45). First, from Eq. (45) we define the generating functional $Z[j, h]$ as

$Z[j, h]=\int[\mathrm{d} \psi][\mathrm{d} \lambda] \mathrm{e}^{j \cdot \psi+h \cdot \lambda-S[\psi, \lambda]}$,

where we have introduced the external source $h$. This allows us to obtain the correlations of the response field $\lambda$ through derivatives with respect to $h$. Next, following Matarrese \& Pietroni (2007a), we add a high- $k$ cutoff $\Lambda$ to the linear power spectrum $P_{\mathrm{L} 0}(k)$ by changing the kernel $\Delta_{\mathrm{I}}$, which appears in the action $S$ of Eq. (46) as

$\Delta_{\mathrm{I}} \rightarrow \Delta_{\Lambda}=\theta\left(\Lambda-k_{1}\right) \Delta_{\mathrm{I}}\left(x_{1}, x_{2}\right)$.

Thus, the Heaviside factor $\theta\left(\Lambda-k_{1}\right)$ removes the linear power at high wavenumbers $k_{1}>\Lambda$, and we recover the full system in the limit $\Lambda \rightarrow \infty$. Then, the idea proposed in Matarrese \& Pietroni (2007a) is to study the evolution of the system as a function of the cutoff $\Lambda$. Therefore, one first looks for equations that describe the dependence on $\Lambda$. Second, one derives some approximation for these equations, for instance by a truncation of some expansion, and finally solves these approximate equations from $\Lambda=0$ up to $\Lambda=\infty$. First, the dependence on $\Lambda$ may obviously be described through the derivative of $Z$ with respect to $\Lambda$, which reads

$\frac{\partial Z}{\partial \Lambda}=\frac{\mathrm{e}^{2 \eta_{\mathrm{I}}} P_{\mathrm{L} 0}(\Lambda)}{2} \int \mathrm{d} \boldsymbol{k} \delta_{\mathrm{D}}(\Lambda-k) \sum_{i, j} \frac{\delta^{2} Z}{\delta h_{i}\left(\boldsymbol{k}, \eta_{\mathrm{I}}\right) \delta h_{j}\left(-\boldsymbol{k}, \eta_{\mathrm{I}}\right)}$.

Next, introducing the generating functional $W$ of the connected correlation functions,

$W=\ln Z, \quad R\left(x_{1}, x_{2}\right)=\left.\frac{\delta^{2} W}{\delta j\left(x_{1}\right) \delta h\left(x_{2}\right)}\right|_{j=h=0}$,

we obtain from Eq. (86) the evolution of the response $R$ with the cutoff $\Lambda$ as

$$
\begin{aligned}
\frac{\partial R}{\partial \Lambda}\left(x_{1}, x_{2}\right)= & \frac{\mathrm{e}^{2 \eta_{\mathrm{I}}} P_{\mathrm{L} 0}(\Lambda)}{2} \int \mathrm{d} \boldsymbol{w} \delta_{\mathrm{D}}(\Lambda-w) \\
& \times \sum_{i, j} \frac{\delta^{4} W}{\delta h_{i}\left(\boldsymbol{w}, \eta_{\mathrm{I}}\right) \delta h_{j}\left(-\boldsymbol{w}, \eta_{\mathrm{I}}\right) \delta j\left(x_{1}\right) \delta h\left(x_{2}\right)} .
\end{aligned}
$$

Here we use the property (47): $\langle\lambda\rangle=\langle\lambda \lambda\rangle=0$. Next, to make some progress, one needs to obtain an expression for the fourthderivative $W^{(4)}$. Of course, in generic cases this quantity is not known exactly and one must introduce some approximations. The usual procedure is to write a diagrammatic expansion for $W$, using the path integral expression (84), and to truncate at some 


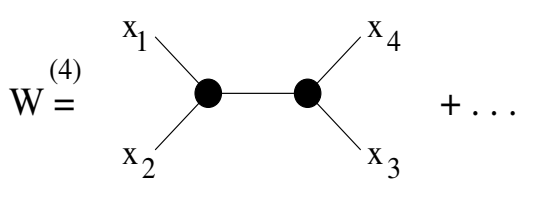

Fig. 1. The first diagram of the expansion of the fourth derivative $W^{(4)}$ as in Eq. (89). The big dots are the vertices $K_{\mathrm{s}}$ and the lines are the two-point functions $R$ or $G$.

finite order. For the cubic action (46), the lowest-order contribution is associated with the diagram of Fig. 1, which gives

$$
\begin{aligned}
\frac{\delta^{4} W}{\delta j_{1} \delta h_{2} \delta h_{3} \delta h_{4}} & =\left\langle\psi_{1} \lambda_{2} \lambda_{3} \lambda_{4}\right\rangle_{c} \\
& =12 R R\left(K_{\mathrm{s}} R K_{\mathrm{s}}\right) R R+\cdots
\end{aligned}
$$

Using expression (20) of the vertex $K_{\mathrm{s}}$, this gives:

$$
\begin{aligned}
& \frac{\partial R_{i_{1} i_{2}}}{\partial \Lambda}\left(k ; \eta_{1}, \eta_{2}\right)=4 \mathrm{e}^{2 \eta_{1}} P_{\mathrm{L} 0}(\Lambda) \int \mathrm{d} w \delta_{\mathrm{D}}(\Lambda-w) \int_{\eta_{2}}^{\eta_{1}} \mathrm{~d} \eta \int_{\eta_{2}}^{\eta} \mathrm{d} \eta^{\prime} \\
& \times R_{i_{1} i_{1}^{\prime}}\left(k ; \eta_{1}, \eta\right) R_{i_{2}^{\prime} i_{2}}\left(k ; \eta^{\prime}, \eta_{2}\right) R_{i_{3}^{\prime} i_{3}}\left(w ; \eta, \eta_{\mathrm{I}}\right) R_{i_{4}^{\prime} i_{4}}\left(w ; \eta^{\prime}, \eta_{\mathrm{I}}\right) \\
& \times R_{i j}\left(\boldsymbol{k}-\boldsymbol{w} ; \eta, \eta^{\prime}\right) \gamma_{i_{1}^{\prime} ; i_{3}^{\prime} i}^{s}(\boldsymbol{w}, \boldsymbol{k}-\boldsymbol{w}) \gamma_{j ; i_{2}^{\prime} i_{4}^{\prime}}^{s}(\boldsymbol{k},-\boldsymbol{w}) .
\end{aligned}
$$

Following Matarrese \& Pietroni (2007a,b), we note that at lowest-order we may replace the response functions on the right hand side in Eq. (90) by the linear responses, which do not depend on $\Lambda$ since they do not depend on the linear power spectrum, see Eq. (30). Then, in the limit $\eta_{\mathrm{I}} \rightarrow-\infty$, only the linear growing modes of $R_{i_{3}^{\prime} i_{3}}$ and $R_{i_{4}^{\prime} i_{4}}$ give a non-vanishing contribution:

$$
\begin{aligned}
\frac{\partial R_{i_{1} i_{2}}}{\partial \Lambda}= & 4 P_{\mathrm{L} 0}(\Lambda) \int \mathrm{d} \boldsymbol{w} \delta_{\mathrm{D}}(\Lambda-w) \int_{\eta_{2}}^{\eta_{1}} \mathrm{~d} \eta \int_{\eta_{2}}^{\eta} \mathrm{d} \eta^{\prime} \mathrm{e}^{\eta+\eta^{\prime}} \\
& \times R_{\mathrm{Li}_{1} i_{1}^{\prime}}\left(\eta_{1}, \eta\right) R_{\mathrm{Li}_{2}^{\prime} i_{2}}\left(\eta^{\prime}, \eta_{2}\right) R_{\mathrm{Lij}}\left(\eta, \eta^{\prime}\right) \\
& \times \gamma_{i_{1}^{\prime} ; i_{3}^{\prime} i}^{s}(\boldsymbol{w}, \boldsymbol{k}-\boldsymbol{w}) \gamma_{j ; i_{2}^{\prime} i_{4}^{\prime}}^{s}(\boldsymbol{k},-\boldsymbol{w}) .
\end{aligned}
$$

Using Eqs. (21), (22) for the vertices $\gamma^{s}$ we obtain:

$$
\frac{\partial R}{\partial \Lambda}=-\frac{4 \pi}{3} k^{2} P_{\mathrm{L} 0}(\Lambda) \frac{\left(D_{1}-D_{2}\right)^{2}}{2} R_{\mathrm{L}}
$$

Starting from the initial condition $R(\Lambda=0)=R_{\mathrm{L}}$, this yields at $\Lambda=\infty$,

$R=R_{\mathrm{L}}\left[1-\frac{\omega^{2}\left(D_{1}-D_{2}\right)^{2}}{2}\right]$,

which agrees with the usual perturbative result at order $P_{\mathrm{L} 0}$ (see Eq. (155) below). The running with $\Lambda$ of the two-point correlation $G$ (whence of the nonlinear power spectrum) is then obtained by taking the derivative with respect to $\Lambda$ of Eq. (83) and again using a loopwise expansion for the self-energy $\Pi$. In practice, Matarrese \& Pietroni (2007a,b) use some ansatz for $G$ to derive a linear equation that can be solved up to $\Lambda=\infty$. Although they refer to this approach as a renormalization group method, we can note that it is somewhat different from the usual renormalization group techniques. Although considering the evolution with a cutoff $\Lambda$, one does not look for a fixed point of renormalization group equations that would govern the properties of the system in some large-scale limit.

Matarrese \& Pietroni (2007a,b) notice that, if we promote the linear response $R_{\mathrm{L}}$ on the right hand side of Eq. (92) to the nonlinear response $R$, we obtain the response (133) with a Gaussian decay at high $k$ as the solution of this linear equation. Note that all the previous steps apply identically to the case of the gravitational dynamics, where this procedure again leads to the response (133). In this case, this expression is no longer exact, but it does show the expected damping into the nonlinear regime. This remark suggests that this procedure may provide a very efficient expansion scheme for the response function. However, this is somewhat artificial. Indeed, to derive Eq. (92) from Eq. (90), one makes use of the properties of the linear response to simplify the right hand side, so that substituting back the nonlinear response $R$ is somewhat ad-hoc (although it is correct at lowestorder it makes the procedure not systematic). Moreover, it is clear that one can apply the same procedure to any other scheme that gives an equation of the form $\partial R / \partial \alpha=F\left[P_{\mathrm{L} 0}, R, G\right]$ where $\alpha$ can be any variable among $\left\{k, \eta_{1}, \eta_{2}, \Lambda, ..\right\}$. (For the large- $N$ expansions of Sect. 4 , it would be $\eta_{1}$.) Indeed, at lowest order one can always simplify $F$ as a linear functional of $R_{\mathrm{L}}$ such that one obtains the exact response (133) by substituting $R_{\mathrm{L}} \rightarrow R$, since the right hand side must be consistent at lowest-order with $\partial R / \partial \alpha$ evaluated for the exact response (133). Thus, the latter satisfies the equation

$\frac{\partial R}{\partial \alpha}=\left[\frac{\partial \ln R_{\mathrm{L}}}{\partial \alpha}-\frac{1}{2} \frac{\partial}{\partial \alpha}\left(D_{1}-D_{2}\right)^{2} \omega^{2}\right] R$,

which implies that at lowest order one can always write $\partial R / \partial \alpha=$ $F\left[P_{\mathrm{L} 0}, R, G\right]$ as

$\left[\frac{\partial}{\partial \alpha}-\frac{\partial \ln R_{\mathrm{L}}}{\partial \alpha}\right] R=-\frac{1}{2}\left(\frac{\partial}{\partial \alpha}\left(D_{1}-D_{2}\right)^{2} \omega^{2}\right) R_{\mathrm{L}}+\cdots$

where the dots stand for higher-order terms over $\left\{\left(D_{1}-D_{2}\right), \omega\right\}$. For the specific case $\alpha=\Lambda$, Eq. (95) leads back to Eq. (92). For the large- $N$ expansion schemes, Eq. (95) would be Eq. (71) with $\alpha \rightarrow \eta_{1}$, the left hand side corresponding to $O \cdot R$ and the right hand side to $\Sigma \cdot R$ at lowest order. Then, substituting $R$ to $R_{\mathrm{L}}$ on the right hand side of Eq. (95), one recovers Eq. (94) and the nonlinear response (133) with the Gaussian cutoff. This reasoning also applies to the gravitational dynamics, to which one adds high- $k$ approximations so that the response (133) (or a variant) still applies; see also Sect. 9 below. Therefore, recovering the response (133) in this manner does not imply that the underlying expansion scheme is very efficient. However, Matarrese \& Pietroni (2007b) argue that for the running with $\Lambda$ it is possible to derive a stronger justification of this procedure which still applies at higher orders. Then, for cases where additional arguments can be obtained, such techniques based on the evolution of the system with respect to some parameter $\alpha$ may provide useful alternative expansion schemes. We discuss this method, based on the dependence of the system on a high- $k$ cutoff $\Lambda$, in Sect. 12 below within the framework of a simple systematic expansion, and we find that it actually gives similar results to the 2PI effective action method.

\section{Exact two-point functions}

For the Zeldovich dynamics, all quantities of interest can be computed exactly, since we know the solution (10) of the equations of motion. This makes the Zeldovich dynamics an interesting test of approximation schemes, since we can compare their predictions with the exact results. As the equations of motion in the form (11), (12) are very similar to those associated with the exact gravitational dynamics, we can expect that the behavior of various approximation schemes will be similar for both dynamics. Therefore, we compute the exact two-point functions associated with the Zeldovich dynamics in this section. 


\subsection{Two-point correlation}

As is well known, the two-point correlation $G$ for the Zeldovich dynamics can be computed exactly from the solution (10) of the equations of motion (e.g. Schneider \& Bartelmann 1995; Taylor $\&$ Hamilton 1996). Indeed, starting from the uniform density $\bar{\rho}$ at $t \rightarrow 0$, the conservation of matter gives, before orbit-crossing,

$\rho(\boldsymbol{x}) \mathrm{d} \boldsymbol{x}=\bar{\rho} \mathrm{d} \boldsymbol{q} \quad$ whence $1+\delta(\boldsymbol{x})=\left[\operatorname{det}\left(\frac{\partial \boldsymbol{x}}{\partial \boldsymbol{q}}\right)\right]^{-1}$.

This also reads from Eq. (10) as

$\delta(\boldsymbol{x}, \eta)=\int \mathrm{d} \boldsymbol{q} \delta_{\mathrm{D}}[\boldsymbol{x}-\boldsymbol{q}-\boldsymbol{s}(\boldsymbol{q}, \eta)]-1$,

where $\boldsymbol{s}(\boldsymbol{q}, \eta)=D_{+}(\eta) \boldsymbol{s}_{\mathrm{L} 0}(\boldsymbol{q})$ is the displacement field. Note that this expression remains valid after shell crossing: all particles of Lagrangian coordinate $\boldsymbol{q}$ that happen to be at location $\boldsymbol{x}$ at the time of interest contribute to the right hand side. In Fourier space we obtain, for $k \neq 0$,

$\delta(\boldsymbol{k})=\int \frac{\mathrm{d} \boldsymbol{q}}{(2 \pi)^{3}} \mathrm{e}^{-\mathrm{i} \boldsymbol{k} \cdot(\boldsymbol{q}+s)}$.

Therefore, the density-density two-point correlation reads as

$$
\begin{aligned}
& \delta_{\mathrm{D}}\left(\boldsymbol{k}_{1}+\boldsymbol{k}_{2}\right) G_{11}\left(k_{1} ; \eta_{1}, \eta_{2}\right)= \\
& \quad \int \frac{\mathrm{d} \boldsymbol{q}_{1} \mathrm{~d} \boldsymbol{q}_{2}}{(2 \pi)^{6}} \mathrm{e}^{-\mathrm{i}\left(\boldsymbol{k}_{1} \cdot \boldsymbol{q}_{1}+\boldsymbol{k}_{2} \cdot \boldsymbol{q}_{2}\right)}\left\langle\mathrm{e}^{-\mathrm{i}\left(\boldsymbol{k}_{1} \cdot \boldsymbol{s}_{1}+\boldsymbol{k}_{2} \cdot \boldsymbol{s}_{2}\right)}\right\rangle .
\end{aligned}
$$

Since the displacement field $s_{\mathrm{L} 0}$ given by Eq. (25) is Gaussian, the average in Eq. (99) reads as

$\left\langle\mathrm{e}^{-\mathrm{i}\left(\boldsymbol{k}_{1} \cdot \boldsymbol{s}_{1}+\boldsymbol{k}_{2} \cdot \boldsymbol{s}_{2}\right)}\right\rangle=\mathrm{e}^{-\frac{1}{2}\left\langle\left(k_{1 i} s_{1 i}+k_{2 i} s_{2 i}\right)\left(k_{1 j} s_{1 j}+k_{2 j} s_{2 j}\right)\right\rangle}$,

where we sum over the $3 \mathrm{D}$ components $i, j=1,2,3$. Let us define the displacement correlation $\Psi_{0}$ :

$\Psi_{0 ; i j}\left(\boldsymbol{q}_{1}, \boldsymbol{q}_{2}\right)=\left\langle s_{\mathrm{L} 0 ; i}\left(\boldsymbol{q}_{1}\right) s_{\mathrm{L} 0 ; j}\left(\boldsymbol{q}_{2}\right)\right\rangle$.

Thanks to statistical homogeneity, it obeys

$\Psi_{0 ; i j}\left(\boldsymbol{q}_{1}, \boldsymbol{q}_{2}\right)=\Psi_{0 ; i j}\left(\boldsymbol{q}_{1}-\boldsymbol{q}_{2}\right)$,

and from Eq. (25) it is given by

$\Psi_{0 ; i j}(\boldsymbol{q})=\int \mathrm{d} \boldsymbol{k} \mathrm{e}^{\mathrm{i} \boldsymbol{k} \cdot \boldsymbol{q}} \frac{k_{i} k_{j}}{k^{4}} P_{\mathrm{L} 0}(k)$.

Then, Eq. (99) writes as

$$
\begin{aligned}
& G_{11}\left(k ; \eta_{1}, \eta_{2}\right)=\int \frac{\mathrm{d} \boldsymbol{q}}{(2 \pi)^{3}} \mathrm{e}^{-\mathrm{i} \boldsymbol{k} \cdot \boldsymbol{q}} \\
& \times \mathrm{e}^{\mathrm{e}^{\eta_{1}+\eta_{2}} k_{i} k_{j}\left[\Psi_{0 ; i j}(\boldsymbol{q})-\cosh \left(\eta_{1}-\eta_{2}\right) \Psi_{0 ; i j}(0)\right]} .
\end{aligned}
$$

Using Eq. (103) this can also be written as

$$
\begin{aligned}
& G_{11}\left(k ; \eta_{1}, \eta_{2}\right)=\int \frac{\mathrm{d} \boldsymbol{q}}{(2 \pi)^{3}} \mathrm{e}^{-\mathrm{i} \boldsymbol{k} \cdot \boldsymbol{q}} \\
& \quad \times \mathrm{e}^{\mathrm{e}^{\eta_{1}+\eta_{2}} \int \mathrm{d} \boldsymbol{w} \frac{(\boldsymbol{k} \cdot \boldsymbol{w})^{2}}{w^{4}} P_{\mathrm{L} 0}(w)\left[\cos (\boldsymbol{w} \cdot \boldsymbol{q})-\cosh \left(\eta_{1}-\eta_{2}\right)\right]} .
\end{aligned}
$$

The integration over angles in the exponent of Eq. (105) can be performed analytically. Thus, let us define the quantity $I(\boldsymbol{q} ; \boldsymbol{k})$ by

$I(\boldsymbol{q} ; \boldsymbol{k})=k_{i} k_{j} \Psi_{0 ; i j}(\boldsymbol{q})=\int \mathrm{d} \boldsymbol{w} \mathrm{e}^{\mathrm{i} w \cdot \boldsymbol{q}} \frac{(\boldsymbol{k} \cdot \boldsymbol{w})^{2}}{w^{4}} P_{\mathrm{L} 0}(w)$.
Then, by expanding the exponential over spherical harmonics, we obtain

$I(\boldsymbol{q} ; \boldsymbol{k})=k^{2} I_{0}(q)+k^{2}\left(1-3 \mu^{2}\right) I_{2}(q), \quad \mu=\frac{\boldsymbol{k} \cdot \boldsymbol{q}}{k q}$,

where we introduce

$I_{n}(q)=\frac{4 \pi}{3} \int_{0}^{\infty} \mathrm{d} w P_{\mathrm{L} 0}(w) j_{n}(w q)$,

and $j_{n}$ is the spherical Bessel function of order $n$. Note that the variance $\sigma_{v}^{2}$ of the one-dimensional displacement field, defined in Eq. (77), also satisfies $\sigma_{v}^{2}=I_{0}(0)$. Then, Eq. (105) reads (using the linear growth factor $D=\mathrm{e}^{\eta}$ as the time-coordinate) as

$$
\begin{aligned}
G_{11}\left(k ; D_{1}, D_{2}\right)= & \mathrm{e}^{-\frac{D_{1}^{2}+D_{2}^{2}}{2} k^{2} \sigma_{v}^{2}} \int \frac{\mathrm{d} \boldsymbol{q}}{(2 \pi)^{3}} \cos (k q \mu) \\
& \times \mathrm{e}^{D_{1} D_{2} k^{2}\left[I_{0}+\left(1-3 \mu^{2}\right) I_{2}\right]} .
\end{aligned}
$$

Following Schneider \& Bartelmann (1995), we can perform the integration over angles by expanding the exponential and using the property

$\int_{0}^{1} \mathrm{~d} \mu \cos (k q \mu)\left(1-\mu^{2}\right)^{n}=n !\left(\frac{2}{k q}\right)^{n} j_{n}(k q)$.

This gives

$$
\begin{aligned}
G_{11}\left(k ; D_{1}, D_{2}\right)= & \mathrm{e}^{-\frac{D_{1}^{2}+D_{2}^{2}}{2} k^{2} \sigma_{v}^{2}} \int_{0}^{\infty} \frac{\mathrm{d} q q^{2}}{2 \pi^{2}} \mathrm{e}^{D_{1} D_{2} k^{2}\left(I_{0}-2 I_{2}\right)} \\
& \times \sum_{n=0}^{\infty}\left(D_{1} D_{2} \frac{6 k^{2} I_{2}}{k q}\right)^{n} j_{n}(k q) .
\end{aligned}
$$

\subsection{Asymptotic behavior}

From the explicit expression (109), we can obtain the asymptotic behavior of the two-point correlation function in the highly nonlinear regime. Thus, we can formally write for a power-law linear power spectrum

$G_{11}=\mathrm{e}^{\left[D_{1} D_{2}-\frac{D_{1}^{2}+D_{2}^{2}}{2}\right] k^{2} \sigma_{v}^{2}} \frac{1}{4 \pi k^{3}} F\left[\Delta_{\mathrm{L}}^{2}\left(k ; D_{1}, D_{2}\right)\right]$

with

$F(x)=\int \frac{\mathrm{d} \boldsymbol{q}}{2 \pi^{2}} \cos (q \mu) \mathrm{e}^{\frac{x}{3} \int \mathrm{d} w w^{n}\left[j_{0}(q w)-1+\left(1-3 \mu^{2}\right) j_{2}(q w)\right]}$

where we make the change of variables $q \rightarrow q / k, w \rightarrow k w$, for

$P_{\mathrm{L} 0}(k)=\frac{1}{4 \pi k_{0}^{3}}\left(\frac{k}{k_{0}}\right)^{n}$,

whence

$\Delta_{\mathrm{L}}^{2}\left(k ; D_{1}, D_{2}\right)=D_{1} D_{2}\left(\frac{k}{k_{0}}\right)^{n+3}$.

First, we note that infrared (IR) divergences appear in the onedimensional velocity dispersion $\sigma_{v}^{2}$ (defined in Eq. (77)) for $n \leq$ -1 at low $k$, and in the integral over $w$ in Eq. (113) for $n \leq-3$. As is well-known, the IR divergence at $n \leq-1$ should disappear for equal-time statistics (Vishniac 1983; Jain \& Bertschinger 1996) because of Galilean invariance. This is explicitly checked in Eq. (112) since for $D_{1}=D_{2}$ the prefactor of $k^{2} \sigma^{2}$ vanishes so that the contribution associated with $\sigma_{v}$ cancels out. Thus the 
equal-time nonlinear power spectrum is well-defined for $n>-3$. Second, we can see that both $\sigma_{v}^{2}$ and the integral over $w$ in Eq. (113) diverge if $n \geq-1$ at high $k$. Thus, this UV divergence remains untamed in the full non-perturbative result (112). This is a qualitative difference with the true gravitational dynamics where such UV divergences are expected to disappear in the exact nonlinear power spectrum for $-3<n<1$. However, this may require going beyond the single-stream approximation. Therefore, in the following we assume $-3<n<-1$. After performing the integral over $w$ and making a change of variable, we obtain

$$
\begin{aligned}
F(x)= & x^{\frac{3}{n+1}} \frac{2}{\pi} \int_{0}^{\infty} \mathrm{d} q q^{2} \int_{0}^{1} \mathrm{~d} \mu \cos \left(x^{\frac{1}{n+1}} q \mu\right) \\
& \times \exp \left[-q^{-n-1} \frac{\pi^{1 / 2} 2^{n-1} \Gamma[(n+3) / 2]}{(-n-1) \Gamma[(4-n) / 2]}\left[1-(n+1) \mu^{2}\right]\right],
\end{aligned}
$$

which shows that $F(x)$ is well-defined for $-3<n<-1$ and obeys the asymptotic behavior

$F(x) \sim x^{\frac{3}{n+1}}$ for $x \gg 1$.

Thus, the equal-time power $\Delta^{2}(k ; D)$ decreases in the highly nonlinear regime as

$\Delta^{2}(k ; D) \sim \Delta_{\mathrm{L}}^{2}(k ; D)^{\frac{3}{n+1}}$ for $\Delta_{\mathrm{L}}^{2} \gg 1$.

Therefore, if $P_{\mathrm{L} 0}(k) \sim k^{n}$ at high $k$ the nonlinear power decreases as a power law $P(k) \sim k^{-3+3(n+3) /(n+1)}$ in the highly nonlinear regime.

\subsection{Response function}

Using the exact solution (10) we can also compute the exact response function $R$. First, we note that, since the velocity field is decoupled from the density field, we have the simple exact result:

$R_{21}=0$.

Of course, the linear solution (30) is consistent with Eq. (119). Next, we can compute the response $R_{12}$ of the density to a velocity perturbation as follows. At time $\eta_{2}^{-}$, before the velocity perturbation localized at time $\eta_{2}$, the location and velocity of the particle of Lagrangian coordinate $\boldsymbol{q}$ are from Eq. (10),

$\boldsymbol{x}_{2}^{-}=\boldsymbol{q}+D_{2} \boldsymbol{s}_{\mathrm{L} 0}(\boldsymbol{q}), \quad \boldsymbol{v}_{2}^{-}=\dot{D}_{2} \boldsymbol{s}_{\mathrm{L} 0}(\boldsymbol{q})$,

whereas at time $\eta_{2}^{+}$, after the velocity perturbation $\zeta_{2}(\boldsymbol{x})$, we have:

$\boldsymbol{x}_{2}^{+}=\boldsymbol{x}_{2}^{-}, \quad \boldsymbol{v}_{2}^{+}=\boldsymbol{v}_{2}^{-}-f_{2} \mathcal{H}_{2} \nabla_{\boldsymbol{x}_{2}}^{-1} \cdot \zeta_{2}$

where we have used the definition of $\psi_{2}$ in Eq. (15). Therefore, the location of the particle at time $\eta_{1}>\eta_{2}$ is

$\boldsymbol{x}_{1}=\boldsymbol{q}+D_{1} s_{\mathrm{L} 0}(\boldsymbol{q})-\frac{D_{1}-D_{2}}{D_{2}} \nabla_{\boldsymbol{x}_{2}}^{-1} \cdot \zeta_{2}$.

The density contrast is again given by expressions of the form (96)-(98) so that $\psi_{1}\left(\boldsymbol{k}_{1}, \eta_{1}\right)=\delta\left(\boldsymbol{k}_{1}, \eta_{1}\right)$ reads for $k_{1} \neq 0$ as

$\psi_{1}\left(\boldsymbol{k}_{1}, \eta_{1}\right)=\int \frac{\mathrm{d} \boldsymbol{q}}{(2 \pi)^{3}} \mathrm{e}^{-\mathrm{i} \boldsymbol{k}_{1} \cdot\left[\boldsymbol{q}+D_{1} s_{\mathrm{L} 0}-\frac{D_{1}-D_{2}}{D_{2}} \nabla_{\boldsymbol{x}_{2}}^{-1} \cdot \zeta_{2}\right]}$.

Definition (28) of the response function reads here as

$R_{12}\left(\boldsymbol{k}_{1}, \eta_{1} ; \boldsymbol{k}_{2}, \eta_{2}\right)=\left\langle\left.\frac{\delta \psi_{1}\left(\boldsymbol{k}_{1}\right)}{\delta \zeta_{2}\left(\boldsymbol{k}_{2}\right)}\right|_{\zeta_{2}=0}\right\rangle$.
Then, using the expression

$-\nabla_{\boldsymbol{x}_{2}}^{-1} \cdot \zeta_{2}=\int \mathrm{d} \boldsymbol{k} \mathrm{e}^{\mathrm{i} \boldsymbol{k} \cdot \boldsymbol{x}_{2}} \mathrm{i} \frac{\boldsymbol{k}}{k^{2}}, \zeta_{2}(\boldsymbol{k})$

we obtain from Eq. (123)

$$
\begin{aligned}
R_{12}= & \frac{D_{1}-D_{2}}{D_{2}} \frac{\boldsymbol{k}_{1} \cdot \boldsymbol{k}_{2}}{k_{2}^{2}} \int \frac{\mathrm{d} \boldsymbol{q}}{(2 \pi)^{3}} \mathrm{e}^{\mathrm{i}\left(\boldsymbol{k}_{2}-\boldsymbol{k}_{1}\right) \cdot \boldsymbol{q}} \\
& \times\left\langle\mathrm{e}^{-\mathrm{i}\left(D_{1} \boldsymbol{k}_{1}-D_{2} \boldsymbol{k}_{2}\right) \cdot \boldsymbol{s}_{\mathrm{L} 0}(\boldsymbol{q})}\right\rangle .
\end{aligned}
$$

Because of homogeneity, the average in Eq. (126) does not depend on $\boldsymbol{q}$ so that the integral over $\boldsymbol{q}$ yields a Dirac factor $\delta_{\mathrm{D}}\left(\boldsymbol{k}_{1}-\boldsymbol{k}_{2}\right)$. On the other hand, since $\boldsymbol{s}_{\mathrm{L} 0}$ is Gaussian the average can be easily performed as in Sect. 6.1, which gives for $D_{1}>D_{2}$ :

$$
\begin{aligned}
R_{12}\left(k ; D_{1}, D_{2}\right) & =\frac{D_{1}-D_{2}}{D_{2}} \mathrm{e}^{-\frac{1}{2}\left(D_{1}-D_{2}\right)^{2} k^{2} \sigma_{v}^{2}} \\
& =R_{\mathrm{L} 12} \mathrm{e}^{-\frac{1}{2}\left(D_{1}-D_{2}\right)^{2} \omega^{2}},
\end{aligned}
$$

where $R_{\mathrm{L} 12}$ is the linear response from Eq. (30) and we have introduced $\omega(k)=k \sigma_{v}$ as in Eq. (77). The computation of $R_{11}$ proceeds along the same lines. A perturbation $\zeta_{1}\left(x_{2}\right)$ of the density field at time $\eta_{2}$ does not modify the velocity field, and we obtain, for $k_{1} \neq 0$,

$$
\begin{aligned}
& \psi_{1}\left(\boldsymbol{k}_{1}, \eta_{1}\right)=\int \frac{\mathrm{d} \boldsymbol{x}_{2}}{(2 \pi)^{3}}\left[1+\delta\left(\boldsymbol{x}_{2}, \eta_{2}^{+}\right)\right] \mathrm{e}^{-\mathrm{i} \boldsymbol{k}_{1} \cdot \boldsymbol{x}_{1}} \\
& \quad=\int \frac{\mathrm{d} \boldsymbol{x}_{2}}{(2 \pi)^{3}} \mathrm{e}^{-\mathrm{i} \boldsymbol{k}_{1} \cdot \boldsymbol{x}_{1}}\left[\int \mathrm{d} \boldsymbol{q} \delta_{\mathrm{D}}\left[\boldsymbol{x}_{2}-\boldsymbol{q}-D_{2} \boldsymbol{s}_{\mathrm{L} 0}(\boldsymbol{q})\right]+\zeta_{1}\left(\boldsymbol{x}_{2}\right)\right]
\end{aligned}
$$

where we have used Eq. (97) and $\boldsymbol{x}_{1}, \boldsymbol{x}_{2}$ are the locations at times $\eta_{1}, \eta_{2}$ of the particle of Lagrangian coordinate $\boldsymbol{q}$. This gives

$R_{11}=\left\langle\operatorname{det}\left(\frac{\partial \boldsymbol{x}_{2}}{\partial \boldsymbol{q}}\right) \mathrm{e}^{-\mathrm{i}\left(D_{1}-D_{2}\right) \boldsymbol{k} \cdot s_{\mathrm{L} 0}(\boldsymbol{q})}\right\rangle$.

Expanding the determinant, we find that most terms cancel out, and we obtain the simple result:

$R_{11}=R_{\mathrm{L} 11} \mathrm{e}^{-\frac{1}{2}\left(D_{1}-D_{2}\right)^{2} \omega^{2}}$.

In a similar fashion, for $R_{22}$ we can write:

$$
\begin{aligned}
\psi_{2}\left(\boldsymbol{k}_{1}, \eta_{1}\right)= & \int \frac{\mathrm{d} \boldsymbol{q}}{(2 \pi)^{3}} \operatorname{det}\left(\frac{\partial \boldsymbol{x}_{1}}{\partial \boldsymbol{q}}\right) \mathrm{e}^{-\mathrm{i} \boldsymbol{k}_{1} \cdot \boldsymbol{x}_{1}} \int \mathrm{d} \boldsymbol{k} \frac{\boldsymbol{k}_{1} \cdot \boldsymbol{k}}{k^{2}} \\
& \times\left[D_{1} \mathrm{e}^{\mathrm{i} \boldsymbol{k} \cdot \boldsymbol{q}} \delta_{\mathrm{L} 0}(\boldsymbol{k})+\frac{D_{1}}{D_{2}} \mathrm{e}^{\mathrm{i} \boldsymbol{k} \cdot \boldsymbol{x}_{2}} \zeta_{2}(\boldsymbol{k})\right] .
\end{aligned}
$$

The computation is slightly more intricate than for $R_{11}$, since $x_{1}$ also depends on $\zeta_{2}$; however, most terms cancel out and we recover again the same form as in Eqs. (128), (131). Thus, the exact nonlinear response function is merely given by

$R\left(k ; D_{1}, D_{2}\right)=R_{\mathrm{L}} \mathrm{e}^{-\frac{1}{2}\left(D_{1}-D_{2}\right)^{2} \omega^{2}}$,

that is, all linear components are multiplied by the same damping factor. We can see from Eq. (133) that the response function only depends on the linear power spectrum through the linear velocity dispersion $\sigma_{v}^{2}$, and on scale through $\omega^{2}=k^{2} \sigma_{v}^{2}$. This property extends to the self-energy $\Sigma$ which is related to $R$ through Eq. (71). This is a big simplification with respect to the gravitational dynamics, where the response $R$ and the self-energy $\Sigma$ depend on the detailed shape of $P_{\mathrm{L} 0}(k)$, see Valageas (2007). However, even in that case it appears that the behavior of the response function is mostly governed by $\omega^{2}=k^{2} \sigma_{v}^{2}$; see for instance the analysis in Sect. 5.2 of Valageas (2007). This also shows that both dynamics share important features. 


\subsection{Damping self-energy $\Sigma$}

The self-energy $\Sigma$ introduced in Sect. 4 is usually obtained as a series of diagrams from the path integral (66). However, since we know the exact response function $R$, we can directly compute $\Sigma$ from Eq. (71). From the simple result (133), we can see that the matrix structure of $R$, hence of $\Sigma$, is not changed by the nonlinear corrections. Therefore, from Eqs. (76) and (133), we write the exact self-energy $\Sigma$ as

$\Sigma\left(k ; D_{1}, D_{2}\right)=\Sigma_{0} \sigma\left[\omega\left(D_{1}-D_{2}\right)\right]$,

where the matrix $\Sigma_{0}$ was obtained in Eq. (76). To generalize the calculation for future use, we consider a response of the form

$R\left(k ; D_{1}, D_{2}\right)=R_{\mathrm{L}} r(t), \quad t=\omega\left(D_{1}-D_{2}\right), \quad r(0)=1$,

where the constraint $r(0)=1$ comes from Eq. (29). Substituting Eqs. (134)-(135) into Eq. (71) yields

$r^{\prime}(t)=-\int_{0}^{t} \mathrm{~d} t^{\prime} \sigma\left(t-t^{\prime}\right) r\left(t^{\prime}\right)$.

The fact that the system (71) can be reduced to Eq. (136) shows that the scalings (134), (135) are indeed self-consistent. Note that the functions $r(t)$ and $\sigma(t)$ are defined for $t \geq 0$, because of the Heaviside factors $\theta\left(\eta_{1}-\eta_{2}\right)$ in $R$ and $\Sigma$. Then, introducing the Laplace transform

$\tilde{r}(s)=\int_{0}^{\infty} \mathrm{d} t \mathrm{e}^{-s t} r(t)$

we obtain from Eq. (136)

$s \tilde{r}(s)-1=-\tilde{\sigma}(s) \tilde{r}(s)$.

For the exact nonlinear response (133), we have:

$r(t)=\mathrm{e}^{-t^{2} / 2}, \quad \tilde{r}(s)=\sqrt{\frac{\pi}{2}} \mathrm{e}^{s^{2} / 2} \operatorname{erfc}\left(\frac{s}{\sqrt{2}}\right)$,

which gives

$\tilde{\sigma}(s)=\sqrt{\frac{2}{\pi}} \frac{\mathrm{e}^{-s^{2} / 2}}{\operatorname{erfc}(s / \sqrt{2})}-s$,

where $\operatorname{erfc}(x)$ is the complementary error function:

$\operatorname{erfc}(x)=\frac{2}{\sqrt{\pi}} \int_{x}^{\infty} \mathrm{d} t \mathrm{e}^{-t^{2}}$

On the other hand, if we write the expansion of $\sigma(t)$ around $t=0$ as

$t \geq 0: \sigma(t)=\sum_{p=0}^{\infty}(-1)^{p} \sigma_{p} \frac{t^{2 p}}{(2 p) !}$,

we obtain by substituting into Eq. (136)

$\sigma_{p}=(2 p+1) ! !-\sum_{m=1}^{p} \sigma_{p-m}(2 m-1) ! !$

and the first few coefficients are

$\sigma_{0}=1, \sigma_{1}=2, \sigma_{2}=10, \sigma_{3}=74, \sigma_{4}=706, \cdots$

We note that this series also appears in other problems of field theory as the number of Feynman diagrams associated for instance to a cubic complex action with two fields
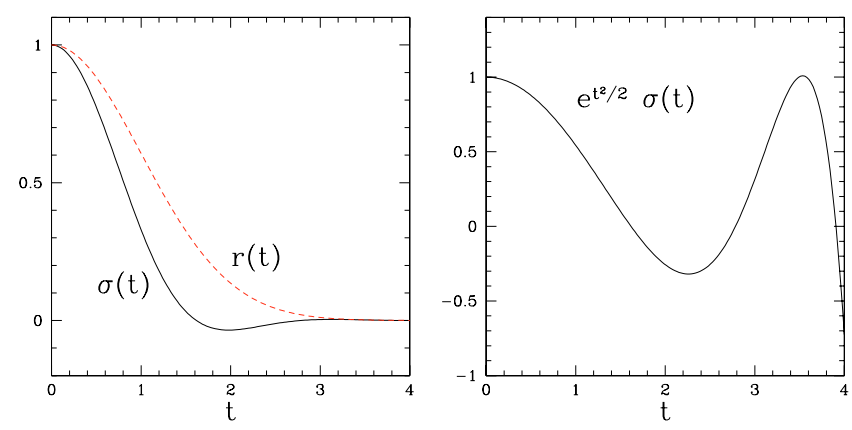

Fig. 2. Left panel: the functions $r(t)$ and $\sigma(t)$. Right panel: the function $\sigma(t)$ multiplied by a factor $\mathrm{e}^{t^{2} / 2}$.

(Cvitanovic et al. 1978). This is not surprising since in our case we also have a cubic action (46) with two fields $\psi, \lambda$. We show in Fig. 2 the behavior of the self-energy function $\sigma(t)$ computed from the expansion (142). We can see that it shows a fast decay, together with oscillations (but the numerical range is too small to check whether the asymptotic is of the form $\left.\mathrm{e}^{-t^{2} / 2} \cos (t)\right)$. Thus, the self-energy exhibits a more intricate behavior than the response $r(t)$. This may explain why path-integral methods based on the Schwinger-Dyson Eq. (71) have difficulty reproducing the response $R$ from simple approximations to $\Sigma$, as we shall see below.

\subsection{Self-energy $\Pi$}

We can also compute the self-energy $\Pi$ from Eq. (83), which gives

$\Pi\left(x_{1}, x_{2}\right)=(O-\Sigma) \cdot G \cdot(O-\Sigma)^{\mathrm{T}}$

using Eqs. (71), (72). Then, using the exact expressions of the two-point correlation $G$ and of the self-energy $\Sigma$, we can obtain $\Pi$. However, using the exact two-point correlation $G_{i j}$ would give intricate expressions, as the velocity correlations involve a few prefactors up to order $P_{\mathrm{L} 0}^{8}$ in front of the exponential of Eq. (105). Here we are mostly interested in the qualitative behavior of the self-energy $\Pi$, therefore, we use the simple approximation

$G \simeq \hat{G}$ with $\hat{G} \equiv G_{11}\left(\begin{array}{ll}1 & 1 \\ 1 & 1\end{array}\right)$,

where $G_{11}$ is the exact density-density correlation derived in Sect. 6.1. The simple form (146) is also consistent with the linear regime limit (27). Then, expanding the exponential of Eq. (109), we can write

$$
\begin{aligned}
\hat{G}\left(k ; D_{1}, D_{2}\right)= & \int \frac{\mathrm{d} \boldsymbol{q}}{(2 \pi)^{3}} \mathrm{e}^{-\mathrm{i} \boldsymbol{k} \cdot \boldsymbol{q}} \sum_{n=1}^{\infty} \frac{1}{n !} I(\boldsymbol{q} ; \boldsymbol{k})^{n} \\
& \times \hat{G}_{n}\left(k, D_{1}\right) \hat{G}_{n}\left(k, D_{2}\right)^{\mathrm{T}}
\end{aligned}
$$

where $I(\boldsymbol{q} ; \boldsymbol{k})$ is defined in Eq. (107), and we introduce the vectors $\hat{G}_{n}$ defined by

$$
\hat{G}_{n}(k, D)=\mathrm{e}^{-\omega^{2} D^{2} / 2} D^{n}\left(\begin{array}{l}
1 \\
1
\end{array}\right) .
$$

In Eq. (147) we use the fact that the term $n=0$ does not contribute to $\hat{G}(k)$. Then, the self-energy $\hat{\Pi}$ associated with $\hat{G}$ 
through Eq. (145) reads as

$$
\begin{aligned}
\hat{\Pi}\left(k ; D_{1}, D_{2}\right)= & \int \frac{\mathrm{d} \boldsymbol{q}}{(2 \pi)^{3}} \mathrm{e}^{-\mathrm{i} \boldsymbol{k} \cdot \boldsymbol{q}} \sum_{n=1}^{\infty} \frac{1}{n !} I(\boldsymbol{q} ; \boldsymbol{k})^{n} \\
& \times \hat{\Pi}_{n}\left(k, D_{1}\right) \hat{\Pi}_{n}\left(k, D_{2}\right)^{\mathrm{T}}
\end{aligned}
$$

with

$\hat{\Pi}_{n}(k, D)=(O-\Sigma) \cdot \hat{G}_{n}=\hat{\pi}_{n}(k, D)\left(\begin{array}{l}1 \\ 1\end{array}\right)$.

Using Eq. (134) we obtain

$$
\begin{aligned}
\hat{\pi}_{n}(k, D) & =\left(n-1-\omega^{2} D^{2}\right) D^{n} \mathrm{e}^{-\omega^{2} D^{2} / 2} \\
& +\omega^{2} D^{2} \int_{0}^{D} \mathrm{~d} D^{\prime} \sigma\left[\omega\left(D-D^{\prime}\right)\right] D^{\prime n-1} \mathrm{e}^{-\omega^{2} D^{\prime 2} / 2} .
\end{aligned}
$$

Then, using Eq. (136) we can check that $\hat{\pi}_{1}=0$. Moreover, if we apply the response $R$ to $\hat{\Pi}$ as in Eq. (83), we obtain obviously from Eqs. (150) and (72)

$$
\begin{aligned}
R \cdot \hat{\Pi} \cdot R^{\mathrm{T}}= & \int \frac{\mathrm{d} \boldsymbol{q}}{(2 \pi)^{3}} \mathrm{e}^{-\mathrm{i} \boldsymbol{k} \cdot \boldsymbol{q}} \sum_{n=2}^{\infty} \frac{1}{n !} I(\boldsymbol{q} ; \boldsymbol{k})^{n} \\
& \times \hat{G}_{n}\left(k, D_{1}\right) \hat{G}_{n}\left(k, D_{2}\right)^{\mathrm{T}},
\end{aligned}
$$

and we recover the full two-point correlation $\hat{G}$ by noticing that the term $n=1$ in Eq. (147) is equal to $R \times G_{\mathrm{I}} \times R^{\mathrm{T}}$ (with $\eta_{\mathrm{I}} \rightarrow-\infty$ ) in agreement with Eq. (83).

\section{Standard perturbative expansions}

In this section, we describe the usual perturbative expansion over powers of the linear power spectrum $P_{\mathrm{L} 0}$ applied to the Zeldovich dynamics. In this article we are considering a $\Lambda \mathrm{CDM}$ universe with $\Omega_{\mathrm{m}}=0.3, \Omega_{\Lambda}=0.7, \Omega_{\mathrm{b}}=0.046, \sigma_{8}=0.9$, a reduced Hubble constant $h=0.7$, and we use the linear power spectrum given by the CAMB Boltzmann code (Lewis et al. 2000). This gives at $z=0$ for a smooth linear power spectrum taken from Eisenstein \& $\mathrm{Hu}(1998)$ :

$\Delta_{\mathrm{L} 0}^{2}\left(k_{0}\right)=1, \quad \omega\left(k_{0}\right)=1.3, \quad$ for $k_{0}=0.21 h \mathrm{Mpc}^{-1}$.

However, until Sect. 13 where we focus on weakly nonlinear scales and baryonic acoustic oscillations, we use a power-law linear power spectrum (114) with $n=-2$ (except in Sect. 10), normalized as in Eq. (153):

$n=-2: \quad \Delta_{\mathrm{L} 0}^{2}(k)=k / k_{0}$,

where $k_{0}$ is given in Eq. (153). This is not important for the response function $R$, which only depends on $\omega(k)=k \sigma_{v}$, whatever the linear power spectrum, but this will allow us to simplify the analysis for the nonlinear two-point correlation $G$. First, we consider the response function $R$. Expanding Eq. (133) over powers of $P_{\mathrm{L} 0}$ is equivalent to expanding over powers of $\omega^{2}$, since $\omega^{2} \propto P_{\mathrm{L} 0}$ from Eq. (77). Therefore, the response function $R^{(p)}$ expanded up to order $P_{\mathrm{L} 0}^{p}$ is:

$R^{(p)}\left(k ; D_{1}, D_{2}\right)=R_{\mathrm{L}} \sum_{m=0}^{p} \frac{(-1)^{m}}{m !}\left[\frac{\omega^{2}}{2}\left(D_{1}-D_{2}\right)^{2}\right]^{m}$.

This expansion converges absolutely at all times and on all scales, but the convergence is not uniform. Thus, the convergence rate is very slow for $\omega\left(D_{1}-D_{2}\right) \gtrsim 1$ and for any finite

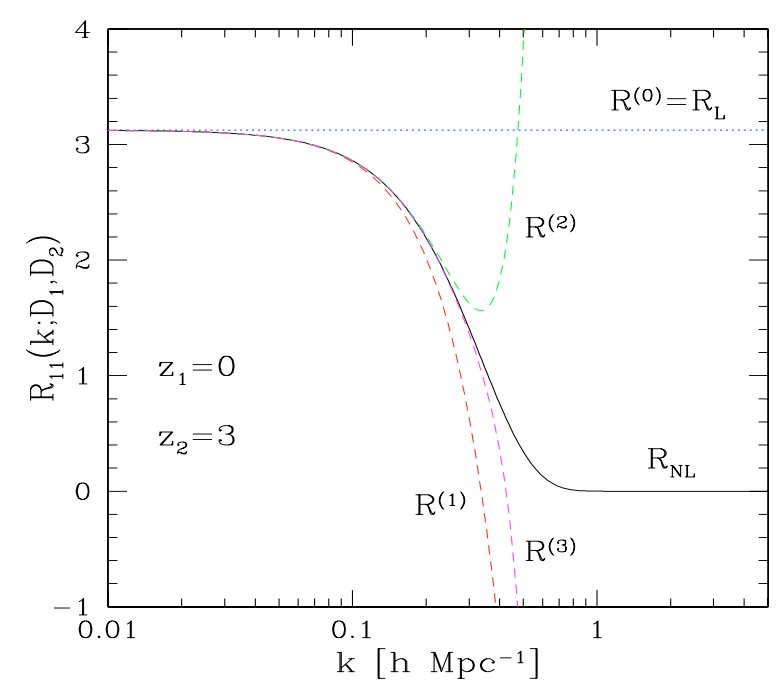

Fig. 3. The standard perturbative expansion of the response function over powers of $P_{\mathrm{L} 0}$ as in Eq. (155). We only show the densitydensity component $R_{11}$ for clarity. The solid curve $R_{\mathrm{NL}}$ is the exact response (133), whereas curves labeled $R^{(p)}$ are the expansion of the response function up to order $p$ over $P_{\mathrm{L} 0}$. Here we consider a $\Lambda \mathrm{CDM}$ Universe with " $n=-2$ " normalized as in Eq. (153), but the results are identical for any CDM cosmology up to a rescaling of $k$.

order $R^{(p)}$ grows without bound at large times or wavenumbers instead of decreasing. From Eq. (155) we can see that in order to obtain a reliable prediction at a given scale we need to go at least up to order $p \sim \omega^{2}\left(D_{1}-D_{2}\right)^{2}$. We display the first few terms in Fig. 3, which clearly shows that increasing the order $p$ improves the agreement with the exact result on weakly nonlinear scales but worsens the prediction in the highly nonlinear regime.

Next, we turn to the standard perturbative expansion of the two-point density-density correlation $G_{11}$. For illustrative purposes we consider the power-law linear power spectrum (154). In this case we can perform the integrals in Eq. (116) and for the equal-time nonlinear power we obtain:

$\Delta^{2}=F\left(\Delta_{\mathrm{L}}^{2}\right)$ with $F(x)=x \tilde{F}(x \pi / 8)$

and

$$
\begin{gathered}
\tilde{F}(y)=\frac{1}{\left(1+y^{2}\right)^{2}}+\frac{3 y\left(1+\sqrt{1+y^{2}}\right) \operatorname{Arctan} \frac{y}{\sqrt{2+y^{2}+2 \sqrt{1+y^{2}}}}}{4\left(1+y^{2}\right)^{5 / 2 \sqrt{2+y^{2}+2 \sqrt{1+y^{2}}}}} \\
+\frac{3 y\left(-1+\sqrt{1+y^{2}}\right) \operatorname{Arctan} \frac{y}{\sqrt{2+y^{2}-2 \sqrt{1+y^{2}}}}}{4\left(1+y^{2}\right)^{5 / 2} \sqrt{2+y^{2}-2 \sqrt{1+y^{2}}}} .
\end{gathered}
$$

Note that the last two terms have not been correctly written in Taylor \& Hamilton (1996). The expansion over powers of $P_{\mathrm{L} 0}$ corresponds to the expansion of $F(x)$ over powers of $x$, and we obtain

$\Delta^{2}=\Delta_{\mathrm{L}}^{2}+\frac{3 \pi^{2}}{64} \Delta_{\mathrm{L}}^{4}-\frac{\pi^{2}}{32} \Delta_{\mathrm{L}}^{6}-\frac{15 \pi^{4}}{8192} \Delta_{\mathrm{L}}^{8}+\cdots$

Contrary to the response function, we can see from Eq. (157) that this expansion diverges for $\Delta_{L}^{2}>8 / \pi$. Therefore, one cannot describe nonlinear scales from this perturbative expansion, and going to higher orders only improves the predictions for weakly 


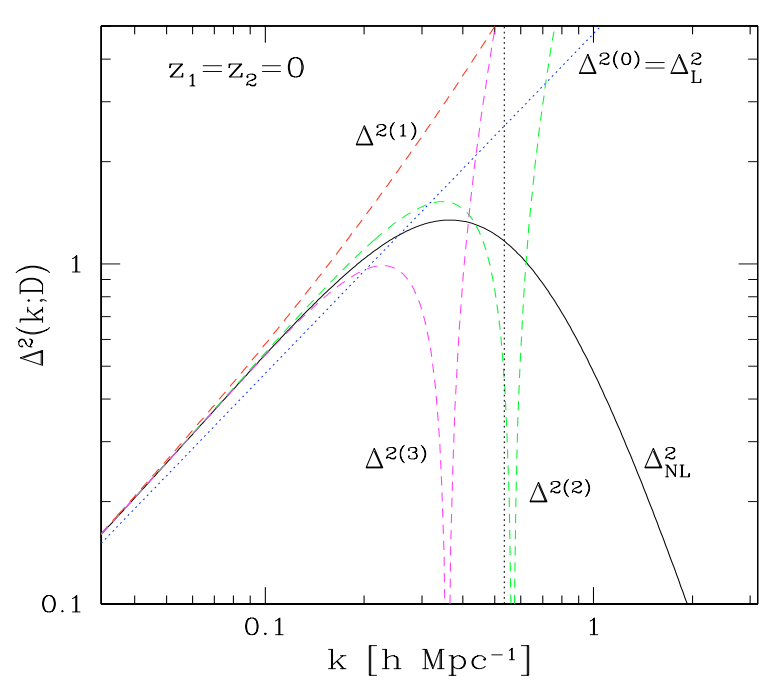

Fig. 4. The standard perturbative expansion of the two-point correlation over powers of $P_{\mathrm{L} 0}$ from Eq. (158). We only show the density-density equal-time logarithmic power $\Delta^{2}(k ; D)$ at redshift $z=0$, for the case $n=-2$. The solid curve $\Delta_{\mathrm{NL}}^{2}$ is the exact power from Eqs. (156), (157), whereas curves labeled $\Delta^{2(p)}$ are the expansion up to order $p+1$ over $P_{\mathrm{L} 0}$. Higher-order terms grow as $k^{p+1}$ at high wavenumbers. The perturbative expansion diverges beyond the vertical dotted line, for $k>$ $0.53 h \mathrm{Mpc}^{-1}$.

nonlinear scales where $\Delta_{\mathrm{L}}^{2}<8 / \pi$. We can see from Eqs. (113)(116) that the radius of convergence of the perturbative series is zero for $n<-2$. (Since at large $q$ we encounter an integrand of the form $\int \mathrm{d} q \mathrm{e}^{-x q^{-n-1}}$, which gives rise to a singularity at $x<0$.) For a $\Lambda \mathrm{CDM}$ cosmology, the slope of the linear power spectrum goes to $n=1$ on large scales; therefore the perturbative series should always converge. However, on small scales where $n \leq-2$ the perturbative expansion is likely to be useless (except for the quasi-linear regime) since the series only converges because of the behavior of the linear power spectrum on unrelated large scales. We compare the first few terms $\Delta^{2(p)}$ of this perturbative expansion with the exact nonlinear power in Fig. 4. In agreement with the analysis above, we can check that the perturbative predictions provide a good match on quasi-linear scales $\Delta_{\mathrm{L}}^{2} \ll 8 / \pi, k \ll 0.53 h \mathrm{Mpc}^{-1}$ and becomes useless deeper into the nonlinear regime.

Crocce \& Scoccimarro (2006a) notice that instead of the standard perturbative expansion (158), one could keep the exponential factor $\mathrm{e}^{-\left(D_{1}^{2}+D_{2}\right)^{2} \omega^{2} / 2}$ in expression (109) and only expand the last exponential $\mathrm{e}^{D_{1} D_{2} I}$. In this fashion, all terms of the new series are positive and damped by the exponential factor at small scales, which gives a seemingly better behaved expansion. We display the results we obtain for the case $n=-2$ in Fig. 5, when we consider the series

$$
\begin{aligned}
\Delta^{2} & =\mathrm{e}^{-D^{2} \omega^{2}}\left[\mathrm{e}^{D^{2} \omega^{2}} F\left(\Delta_{\mathrm{L}}^{2}\right)\right] \\
& =\mathrm{e}^{-D^{2} \omega^{2}}\left[\Delta_{\mathrm{L}}^{2}+\left(\frac{3 \pi^{2}}{64} \Delta_{\mathrm{L}}^{4}+D^{2} \omega^{2} \Delta_{\mathrm{L}}^{2}\right)+\cdots\right]
\end{aligned}
$$

where we use the normalization of Eq. (153) for $\omega(k)$. Of course, for a power-law linear power spectrum, we actually have $\omega=\infty$, but Eq. (159) describes a CDM-like power spectrum, such that $n=-2$ on the weakly nonlinear scales of interest and $\omega$ is made finite and normalized to Eq. (153) through IR and UV cutoffs. Figure 5 shows that indeed the terms at each order over $P_{\mathrm{L} 0}$

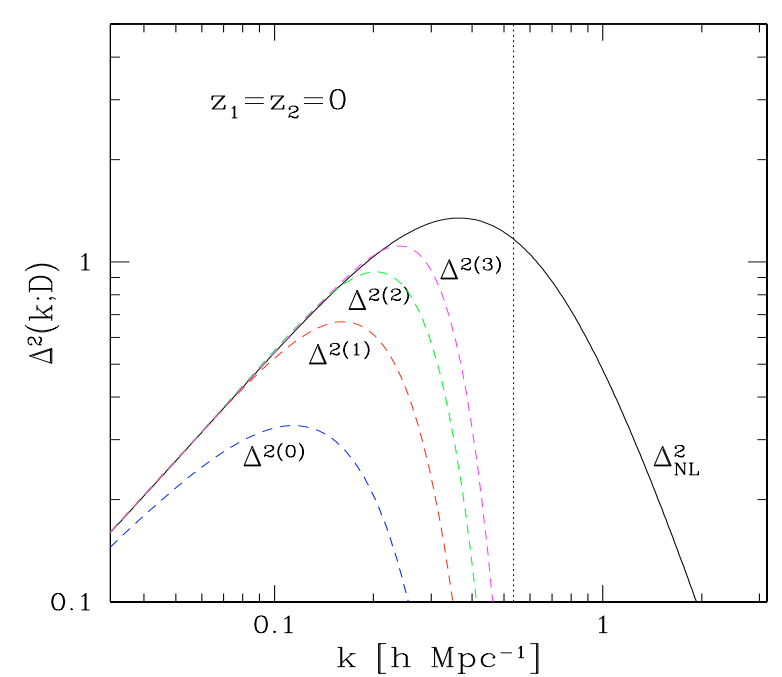

Fig. 5. The perturbative expansion of the two-point correlation over powers of $P_{\mathrm{L} 0}$ from Eq. (159) where we keep the exponential factor $\mathrm{e}^{-D^{2} \omega^{2}}$ apart. The solid curve $\Delta_{\mathrm{NL}}^{2}$ is the exact power from Eqs. (156), (157), whereas curves labeled $\Delta^{2(p)}$ are the expansion up to order $p+1$ over $P_{\mathrm{L} 0}$. All terms decay as $\mathrm{e}^{-D^{2} \sigma_{v}^{2} k^{2}}$ at high $k$. The perturbative expansion again diverges beyond the vertical dotted line, for $k>0.53 h \mathrm{Mpc}^{-1}$.

are positive, and the series looks better behaved. However, it is clear that the series still diverges beyond $k \simeq 0.53 h \mathrm{Mpc}^{-1}$ as for (158). Moreover, we note that increasing $\omega\left(k_{0}\right)$ (by moving the IR and UV cutoffs) would move the Gaussian cutoff towards smaller $k$ and would worsen the agreement with the exact nonlinear power. In fact, for general power-spectra where the linear velocity dispersion $\sigma_{v}^{2}$ is not necessarily governed by the weakly nonlinear scales of interest, the rewriting associated with Eq. (159) does not always improve the agreement with the exact power. Power-law linear power spectra are a clear example of such cases, since $\omega=\infty$ so that the expansion (159) is not well-defined (unless one absorbs IR divergences into a renormalized $\sigma_{v}{ }^{1}$ ), even though the equal-time power remains finite, and the usual perturbative expansion (158) is well-defined. Note that the expansion schemes based on Eq. (83), through path-integral or diagrammatic methods, do not exactly correspond to the expansion (159). Although the first term $R \times G_{\mathrm{I}} \times R^{\mathrm{T}}$ may exhibit the factor $\mathrm{e}^{-D^{2} \omega^{2}}$, this is no longer true for the second term (because of the integrations over time in $R \cdot \Pi \cdot R^{\mathrm{T}}$ ), as shown by the discussion in Sect. 8.3. We note, however, that since the response function $R$ also involves the quantity $\omega$ we can expect such schemes to fail in cases where the velocity dispersion $\sigma_{v}^{2}$ is governed by scales that are very far from those of interest.

\section{Direct steepest-descent method}

\subsection{Response $R$ and damping self-energy $\Sigma$}

We now investigate the properties of the steepest-descent method described in Sect. 4.1. We first consider the damping self-energy

1 The procedure (159) could be modified to apply to cases such as $n=-2$ without any IR cutoff, as the exact expression (112) shows that the IR divergences are fully absorbed into $\omega=k \sigma_{v}$. Then, by splitting all IR-divergent integrals into a $\sigma_{v}$ part and a finite part and using a given value for $\sigma_{v}$, one obtains finite expansions. The value of $\sigma_{v}$ is irrelevant for exact equal-time statistics (but would remain in approximate quantities obtained as in Eq. (159) by truncation at some order). 


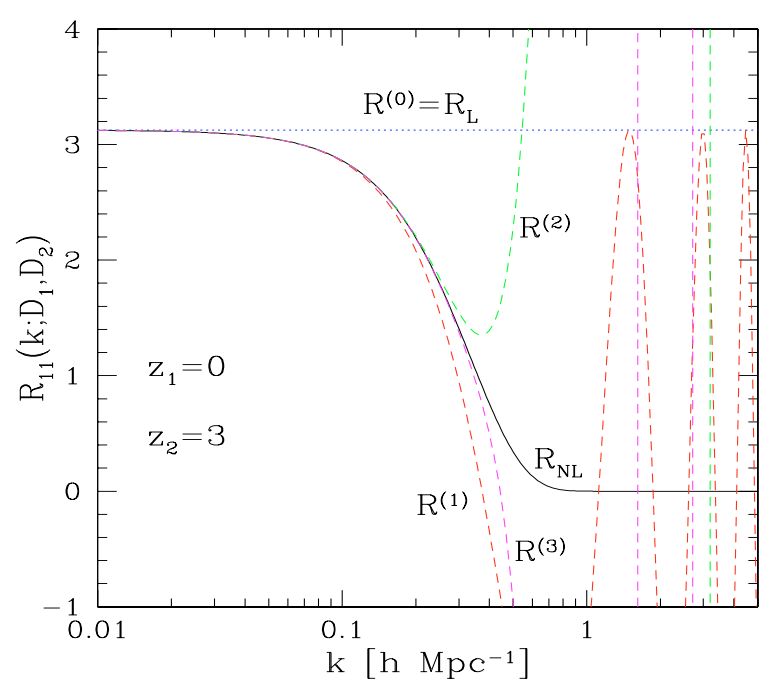

Fig. 6. The expansion of the response function defined by the direct steepest-descent method, from Eq. (162). We only show the densitydensity component $R_{11}$ for clarity. The solid curve $R_{\mathrm{NL}}$ is the exact response (133), whereas curves labeled $R^{(p)}$ are the expansion of the response function up to order $p$.

$\Sigma$ and the response $R$. The self-energy $\Sigma$ can be obtained at oneloop order from Eq. (73), which gives Eq. (76), and at higher orders by including higher-order diagrams. However, for the Zeldovich dynamics, it can be directly obtained from the exact expression derived in Sect. 6.4. From Eq. (134) it is clear that series (142) corresponds to the perturbative expansion of the selfenergy $\Sigma$ over powers of $P_{\mathrm{L} 0}$ (through powers of $\left.\omega^{2}\right)$. The oneloop result (76) simply corresponds to the first term $\sigma^{(1)}(t)=\sigma_{0}$ (whereas the linear regime corresponds to $\sigma=0$ ) because of the prefactor $\omega^{2}$ in $\Sigma_{0}$ defined in Eq. (76). In this fashion we obtain the self-energy $\Sigma$ up to order $p$ as

$\Sigma^{(p)}=\Sigma_{0} \sigma^{(p)}=\Sigma_{0} \sum_{m=0}^{p-1}(-1)^{m} \sigma_{m} \frac{\left[\omega\left(D_{1}-D_{2}\right)\right]^{2 m}}{(2 m) !}$.

This in turn determines the response function through the steepest-descent method described in Sect. 4.1 as

$R^{(p)}\left(k ; D_{1}, D_{2}\right)=R_{\mathrm{L}} r^{(p)}\left[\omega\left(D_{1}-D_{2}\right)\right]$,

where $r^{(p)}(t)$ is obtained from $\sigma^{(p)}(t)$ through Eq. (136). From Eq. (138) this gives

$\tilde{r}^{(p)}(s)=\frac{s^{2 p-1}}{s^{2 p}+\sum_{m=0}^{p-1}(-1)^{m} \sigma_{m} s^{2(p-1-m)}}$.

For the first few terms this gives

$r^{(0)}=1, r^{(1)}=\cos t, r^{(2)}=\frac{1}{3} \cosh t+\frac{2}{3} \cos \sqrt{2} t$.

Indeed, the perturbative expansion of the self-energy $\Sigma$ gives $\sigma(t)$ as a series over powers of $t$, whence $\tilde{\sigma}(s)$ as a series over powers of $1 / s$. This yields a rational function for $\tilde{r}^{(p)}(s)$ and a sum of exponentials for $r^{(p)}(t)$. At order $p=1$, the arguments of the exponentials are imaginary (they are given by the roots of the denominator of $\tilde{r}^{(p)}(s)$ ), but real parts appear at order $p=2,3$ (and presumably at higher orders). Since the denominator is even, see Eq. (162), the roots appear by pairs $\pm s_{i}$ so that both decaying and growing exponentials appear. Therefore, the direct steepest-descent method cannot reproduce the decay of the response function at large $t$ (i.e. in the highly nonlinear regime). Of course, at a given order $p$, the response $r^{(p)}(t)$ agrees with the expansion of the exact response (155) up to order $t^{2 p}$. We display the first few terms $R^{(p)}$ in Fig. 6. The behavior is actually rather close to the standard perturbative expansion shown in Fig. 3, as the higher orders slowly improve the agreements over weakly nonlinear scales but explode increasingly fast into the highly nonlinear regime (but their amplitude grows even faster as exponentials instead of power laws).

\subsection{Padé approximants}

The remarks above suggest that we may improve the expansion (162) by looking for a Padé approximant to the rational function $\tilde{r}^{(p)}(s)$, which has the same expansion over $1 / s$ up to $1 / s^{2 p+1}$. In fact, since we know the exact response function, we can directly obtain the series of Padé approximants from Eq. (139). First, from the expansion of $\mathrm{e}^{-t^{2} / 2}$ at $t=0$, we obtain formally the expansion of $\tilde{r}(s)$ over $1 / s$ as

$\tilde{r}(s)=\sum_{p=0}^{\infty}(-1)^{p}(2 p-1) ! ! s^{-2 p-1}$.

Note that this only provides an asymptotic series for $\tilde{r}(s)$ in the limit $s \rightarrow \infty$. Here it is convenient to make the change of variable $y=2 / s^{2}$ and to define $\bar{r}(y)$ by

$\tilde{r}(s)=\frac{1}{s} \bar{r}\left(y=2 / s^{2}\right), \quad \bar{r}(y)=\sum_{p=0}^{\infty}(-1)^{p} \bar{r}_{p} y^{p}$.

From Eq. (164) we obtain for the coefficients $\bar{r}_{p}$

$\bar{r}_{p}=\frac{(2 p-1) ! !}{2^{p}}=\frac{\Gamma[p+1 / 2]}{\sqrt{\pi}}=\int_{0}^{\infty} \frac{\mathrm{d} t}{\sqrt{\pi t}} \mathrm{e}^{-t} t^{p}$.

This shows that the expansion (165) is a Stieltjes series since the coefficients $\bar{r}_{p}$ are the moments of a real positive function defined over $t \geq 0$ (here of the function $\mathrm{e}^{-t} / \sqrt{\pi t}$ ), see Bender \& Orszag (1978). Since Carleman's condition is fulfilled, $\sum \bar{r}_{p}^{-1 / 2 p}=\infty$, the function $\bar{r}(y)$ is uniquely determined by its asymptotic expansion (165), which can be resummed as

$\bar{r}(y)=\int_{0}^{\infty} \frac{\mathrm{d} t}{1+y t} \frac{\mathrm{e}^{-t}}{\sqrt{\pi t}}$.

Then, all coefficients of the continued-fraction representation of $\bar{r}(y)$ are nonnegative, and both Padé sequences $P_{p}^{p}$ and $P_{p+1}^{p}$ converge monotonically to $\bar{r}(y)$ as

$$
\begin{aligned}
P_{1}^{0}(y) & \leq P_{2}^{1}(y) \leq P_{3}^{2}(y) \leq \cdots \leq \bar{r}(y) \\
\bar{r}(y) & \leq \cdot . \leq P_{2}^{2}(y) \leq P_{1}^{1}(y) \leq P_{0}^{0}(y)
\end{aligned}
$$

and

$\bar{r}(y)=\lim _{p \rightarrow \infty} P_{p+1}^{p}(y)=\lim _{p \rightarrow \infty} P_{p}^{p}(y)$.

By contrast, the usual perturbative expansion (155), which is associated with expansion (164), amounts to approximate $\bar{r}(y)$ by a polynomial, that is, by the sequence $P_{0}^{p}(y)$, whereas the steepestdescent method (162) amounts to approximate $1 / \bar{r}(y)$ by a polynomial, that is, $\bar{r}(y)$ by the sequence $P_{p}^{0}(y)$. As we have seen above, these two sequences do not converge very well since they 


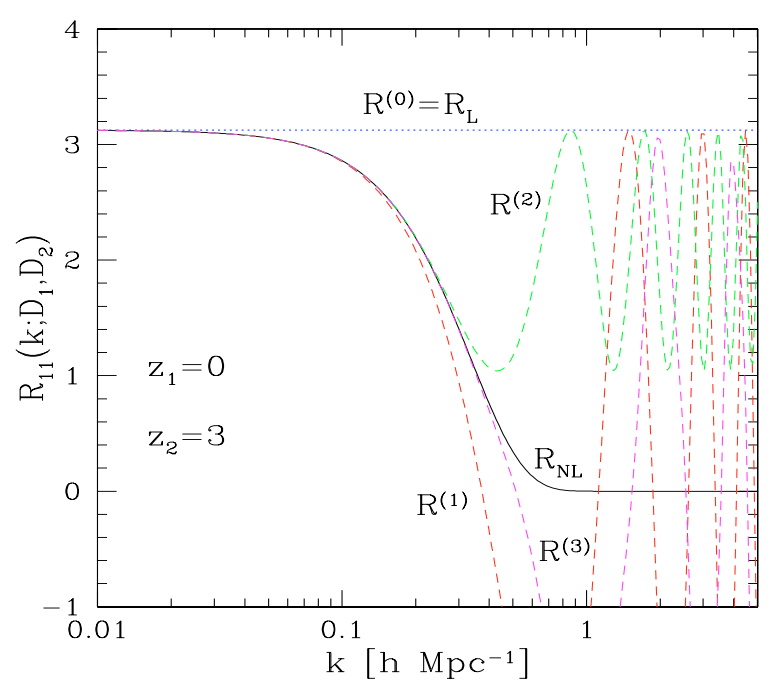

Fig. 7. The expansion of the response function defined by the Padé approximants from Eqs. (171). At each order, one obtains a sum of cosines, with an offset for even $p$.

give a response $r^{(p)}(t)$ that grows without bound for $t \rightarrow \infty$. On the other hand, the sequence associated with (170) gives

$\bar{r}^{(0)}(y)=P_{0}^{0}=1, \bar{r}^{(1)}(y)=P_{1}^{0}=\frac{1}{1+y / 2}$,

$\bar{r}^{(2)}(y)=P_{1}^{1}=\frac{1+y}{1+3 y / 2}, \cdots$

The first two terms give the same results $r^{(0)}$ and $r^{(1)}$ as Eq. (163) but the next two terms give:

$$
\begin{aligned}
r^{(2)}(t)= & \frac{2}{3}+\frac{1}{3} \cos \sqrt{3} t, \\
r^{(3)}(t)= & \frac{\sqrt{6}+2}{2 \sqrt{6}} \cos \sqrt{3-\sqrt{6}} t \\
& +\frac{\sqrt{6}-2}{2 \sqrt{6}} \cos \sqrt{3+\sqrt{6}} t,
\end{aligned}
$$

which do not grow exponentially at large $t$ any more. Because both the sequences $P_{p}^{p}$ and $P_{p+1}^{p}$ and $\bar{r}(y)$ are Stieltjes functions, they are analytic in the cut plane $|\arg (y)|<\pi$, and all poles of the Padé approximants $P_{p}^{p}$ and $P_{p+1}^{p}$ lie on the negative real axis (Bender \& Orszag 1978). Therefore, Eq. (165) shows that all poles of the associated rational function $\tilde{r}^{(p)}(s)$ lie on the imaginary axis and appear by pairs $\pm i s_{j}$ together with a pole at $s=0$ for even $p$. Then, the response factor $r^{(p)}(t)$ is a sum of cosines $\cos \left(s_{j} t\right)$ plus a constant for even $p$, in agreement with Eqs. (172), (173). This is a clear improvement over the standard expansion (155) and the direct steepest-descent expansion (162), (163). However, even this expansion cannot recover the Gaussian decay $\mathrm{e}^{-t^{2} / 2}$, which is replaced by fast oscillations. Nevertheless, this gives rise to an effective damping (for odd $p$ ) once the response function is integrated with some weight function. We compare the first few terms to the exact nonlinear response in Fig. 7. Of course, we recover the same agreement at low $k$ as with the other expansions displayed in Figs. 3 and 6, but as explained above, the response remains bounded at high $k$ with fast oscillations.

\subsection{Correlation $G$ and self-energy $\Pi$}

We now consider the predictions of the direct steepest-descent method for the two-point correlation $G$ and self-energy $\Pi$. As in Sect. 7, we consider the case of a power-law linear power spectrum $n=-2$, which simplifies the calculations, since integrals over wavenumber $k$ have already been performed following Eqs. (156)-(157). Moreover, as in Eq. (159) and Fig. 5, we consider a finite linear velocity dispersion $\omega(k)=k \sigma_{v}$ normalized in Eq. (153). As in Sect. 6.5, in order to simplify the computations, we approximate the matrix form of the correlation $G_{i j}$ by Eq. (146). Thus, we keep the exact nonlinear, density-density correlation $G_{11}$, and make approximation $G_{i j}=G_{11}$ for all $\{i, j\}$. Using Eq. (112) and expanding the exponential $\mathrm{e}^{D_{1} D_{2} \omega^{2}}$ and $F(x)$, we write $G_{11}$ as

$G_{11}=\frac{1}{4 \pi k^{3}} \sum_{p, m=0}^{\infty} \frac{F_{p}}{p ! m !} \Delta_{\mathrm{L} 0}^{2 p} \omega^{2 m}\left(D_{1} D_{2}\right)^{p+m} \mathrm{e}^{-\frac{D_{1}^{2}+D_{2}^{2}}{2} \omega^{2}}$

where $F_{p}$ is the $p$ th derivative of $F(x)$ at $x=0$ :

$F(x)=\sum_{p=0}^{\infty} \frac{F_{p}}{p !} x^{p}$

The first few coefficients $F_{p}$ are given in Eq. (158). Note, however, that for the case $n=-2$, the Taylor series (175) diverges for $x>8 / \pi$ as seen in Sect. 7. Then, we can write the matrix two-point correlation $\hat{G}$ as

$\hat{G}=\frac{1}{4 \pi k^{3}} \sum_{p, m} \frac{F_{p}}{p ! m !} \Delta_{\mathrm{L} 0}^{2 p} \omega^{2 m} \hat{G}_{p+m}\left(D_{1}\right) \hat{G}_{p+m}\left(D_{2}\right)^{\mathrm{T}}$,

where the vectors $\hat{G}_{n}$ have been defined in Eq. (148). Thus, as compared with Eq. (147), the use of a power-law linear power spectrum has simply replaced the integral over $\boldsymbol{q}$ by a discrete sum. Then Eqs. (149)-(152) still apply, once we replace the integral over $\boldsymbol{q}$ by this discrete sum. We now need to compute the functions $\hat{\pi}_{n}(D)$ defined in Eq. (151). Using the expansion (142), we can perform the integrations over $D^{\prime}$ and obtain

$$
\begin{aligned}
& \hat{\pi}_{n}(D)=(n-1) D^{n}+D^{n} \sum_{m=0}^{\infty} \frac{(-1)^{m}}{m !}\left(\frac{\omega^{2} D^{2}}{2}\right)^{m+1} \\
& \times\left[-\frac{n-1}{m+1}-2+\frac{m ! 2^{m+1}}{(n+2 m) !} \sum_{\ell=0}^{m} \sigma_{m-\ell} \frac{(n+2 \ell-1) !}{\ell ! 2^{\ell}}\right] .
\end{aligned}
$$

Of course, from Eq. (143) we can check that $\hat{\pi}_{1}=0$, in agreement with the result already obtained in Sect. 6.5. Substituting Eq. (177) into the analog of Eq. (149), which reads here (as Eq. (176) for $\hat{G}$ ) as

$\hat{\Pi}=\frac{1}{4 \pi k^{3}} \sum_{p, m} \frac{F_{p}}{p ! m !} \Delta_{\mathrm{L} 0}^{2 p} \omega^{2 m} \hat{\Pi}_{p+m}\left(D_{1}\right) \hat{\Pi}_{p+m}\left(D_{2}\right)^{\mathrm{T}}$

we obtain the self-energy $\hat{\Pi}$ up to order $P_{\mathrm{L} 0}^{p+1}$ as

$\hat{\Pi}^{(p)}\left(k ; D_{1}, D_{2}\right)=\frac{1}{4 \pi k^{3}} \sum_{n=1}^{p} \hat{\Pi}_{n}\left(k ; D_{1}, D_{2}\right)\left(\begin{array}{cc}1 & 1 \\ 1 & 1\end{array}\right)$,

where $\hat{\Pi}_{n} \propto P_{\mathrm{L} 0}^{n+1}$ and the first few terms are

$\hat{\Pi}_{1}=\Delta_{\mathrm{L}}^{2}\left(\frac{3 \pi^{2}}{64} \Delta_{\mathrm{L}}^{2}+\omega_{1} \omega_{2}\right)$ 


$$
\begin{aligned}
\hat{\Pi}_{2}= & -\Delta_{\mathrm{L}}^{2}\left(\omega_{1}^{2}+\omega_{2}^{2}\right)\left(\frac{3 \pi^{2}}{64} \Delta_{\mathrm{L}}^{2}+\omega_{1} \omega_{2}\right) \\
& +\Delta_{\mathrm{L}}^{2}\left(-\frac{\pi^{2}}{8} \Delta_{\mathrm{L}}^{4}+\frac{3 \pi^{2}}{16} \Delta_{\mathrm{L}}^{2} \omega_{1} \omega_{2}+2 \omega_{1}^{2} \omega_{2}^{2}\right)
\end{aligned}
$$

where we have introduced (we recall that $\Delta_{\mathrm{L}}^{2}=D_{1} D_{2} \Delta_{\mathrm{L} 0}^{2}$ )

$\omega_{1}=D_{1} \omega(k)=D_{1} k \sigma_{v}, \quad \omega_{2}=D_{2} \omega(k)$.

As for the standard perturbative expansion (158) applied to the correlation function $G$, the series expansion (179) for the selfenergy $\hat{\Pi}$ gives higher-order terms that grow increasingly fast into the highly nonlinear regime. Moreover, we can expect that it diverges at equal times for $\Delta_{\mathrm{L}}^{2}>8 / \pi$ as for $G$.

Then, the direct-steepest descent method prediction at order $p$ is obtained by applying Eq. (83) using the response $R^{(p)}$ and the self-energy $\hat{\Pi}^{(p)}$ at that order. Note that this expansion does not have the form of a standard perturbative expansion such as Eqs. (158) or (159), since all terms in the series get modified as we go to higher orders, because the response $R^{(p)}$ has a different functional form, see (163). At linear order $p=0$ we have $\Pi=0$ and $\Delta^{2(0)}=\Delta_{\mathrm{L}}^{2}$. At first order $p=1$, we obtain from Eq. (163)

$$
\begin{aligned}
& \Delta^{2(1)}\left(k ; D_{1}, D_{2}\right)=\Delta_{\mathrm{L}}^{2} \cos \left(\omega_{1}\right) \cos \left(\omega_{2}\right) \\
& +\Delta_{\mathrm{L}}^{2}\left(\frac{3 \pi^{2}}{64} \Delta_{\mathrm{L}}^{2}+\omega_{1} \omega_{2}\right) \frac{\sin \left(\omega_{1}\right) \sin \left(\omega_{2}\right)}{\omega_{1} \omega_{2}} .
\end{aligned}
$$

Of course, if we expand Eq. (183) over powers of $\omega$, we recover the usual perturbative result, and at equal time we recover Eq. (158) up to order $\Delta_{\mathrm{L}}^{4}$. Moreover, we already know that at equal times $D_{1}=D_{2}$, all terms $\omega$ must cancel out up to order $P_{\mathrm{L} 0}^{p+1}$ because the exact power (156) does not depend on $\omega$. This is a general result that does not depend on the shape of the linear power spectrum and can be seen from Eq. (105). This is related to the cancellation of IR divergences recalled in Sect. 6.2 associated with Galilean invariance. In a similar fashion, at order $p=2$, we obtain from Eq. (163)

$$
\begin{aligned}
\Delta^{2(2)}= & \Delta_{\mathrm{L}}^{2} r_{1}^{(2)} r_{2}^{(2)}+\Delta_{\mathrm{L}}^{2}\left(\frac{3 \pi^{2}}{64} \Delta_{\mathrm{L}}^{2}+\omega_{1} \omega_{2}\right) r_{1}^{(2 ; 1)} r_{2}^{(2 ; 1)} \\
& -\Delta_{\mathrm{L}}^{2}\left(\frac{3 \pi^{2}}{64} \Delta_{\mathrm{L}}^{2}+\omega_{1} \omega_{2}\right)\left(\omega_{1}^{2} r_{1}^{(2 ; 3)} r_{2}^{(2 ; 1)}+\omega_{2}^{2} r_{1}^{(2 ; 1)} r_{2}^{(2 ; 3)}\right) \\
& +\Delta_{\mathrm{L}}^{2}\left(-\frac{\pi^{2}}{8} \Delta_{\mathrm{L}}^{4}+\frac{3 \pi^{2}}{16} \Delta_{\mathrm{L}}^{2} \omega_{1} \omega_{2}+2 \omega_{1}^{2} \omega_{2}^{2}\right) r_{1}^{(2 ; 2)} r_{2}^{(2 ; 2)}
\end{aligned}
$$

where we have defined $(\ell \geq 1)$

$r_{i}^{(p)}=r^{(p)}\left(\omega_{i}\right), \quad r_{i}^{(p ; \ell)}=\int_{0}^{1} \mathrm{~d} t t^{\ell-1} r^{(p)}\left[\omega_{i}(1-t)\right]$.

We display our results for the first few terms in Fig. 8. We can see that the exponential terms associated with the response function (see Eq. (163)) make the higher-order approximations grow exponentially at high $k$ (for $p \geq 2$ ). This very strong growth makes the series of little practical value for $p \geq 2$, where the convergence is not better than for the standard perturbative expansion displayed in Fig. 4. We note that the equal-time power can become negative at high $k$, whereas both $\Pi$ and $G$ should be positive matrices from Eq. (83), which implies that the components $G_{i i}$ and $\Pi_{i i}$ are positive at equal times. This failure comes from the truncation of the self-energy $\hat{\Pi}$ in Eq. (179), which breaks the positivity of $\hat{\Pi}$. Indeed, from Eq. (181) and the scalings $\Delta_{\mathrm{L}}^{2} \propto k$ and $\omega \propto k$, we see that $\hat{\Pi}_{2} \sim-3 \pi^{2} / 32 \Delta_{\mathrm{L}}^{4} \omega^{2}$ at high $k$.

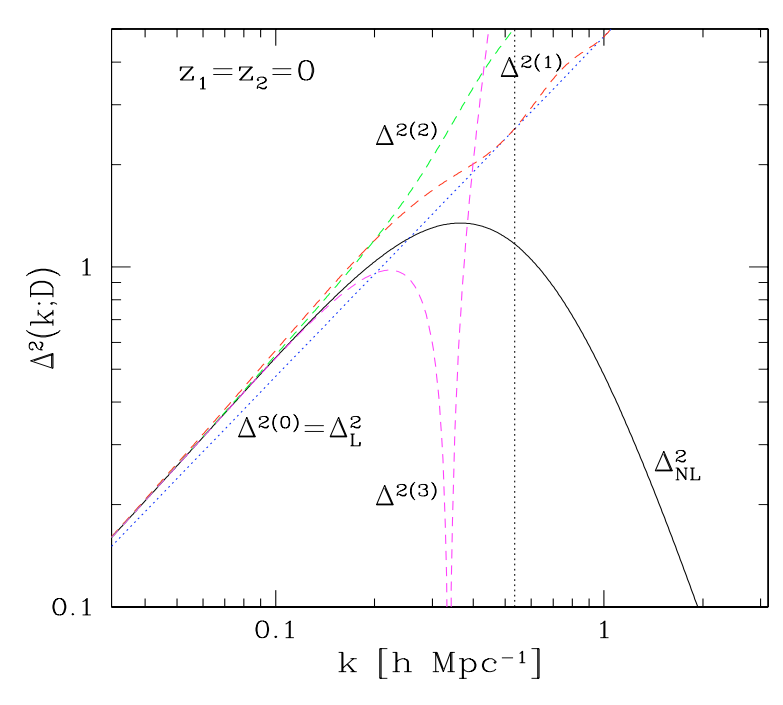

Fig. 8. The expansion of the two-point correlation defined by the direct steepest-descent method, from Eq. (179). Higher-order terms display an exponential growth at high $k$ for $p \geq 2$.

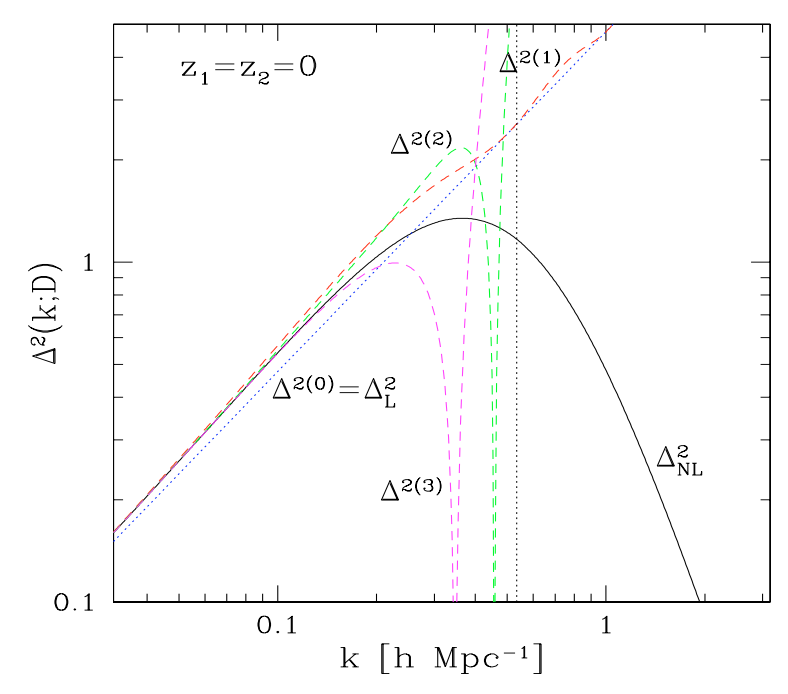

Fig. 9. The expansion of the two-point correlation defined by the direct steepest-descent method, from Eq. (179), but using the Padé approximants (172), (173) for the response function. Higher-order terms display a power-law growth at high $k$.

We show in Fig. 9 the results obtained from the expansion (179) for the self-energy $\hat{\Pi}$ but using the Padé approximants (172), (173) for the response function. The higher-order terms no longer grow as exponentials at high $k$ but as power laws, since the response functions only contain constants and cosines instead of exponentials. However, this is not sufficient for significantly improving the convergence of the series to the exact nonlinear power $\Delta^{2}$.

Finally, we show in Fig. 10 the results obtained from the expansion (179) for the self-energy $\hat{\Pi}$ when we use the exact response function (133). Note that in this case the lowestorder approximation $\Delta^{2(0)}$ is equal to the linear power multiplied by a Gaussian damping factor. It is actually equal to the first term of Eq. (159). Higher-order terms do not exhibit such a Gaussian damping because of the time-integrals involved in the 


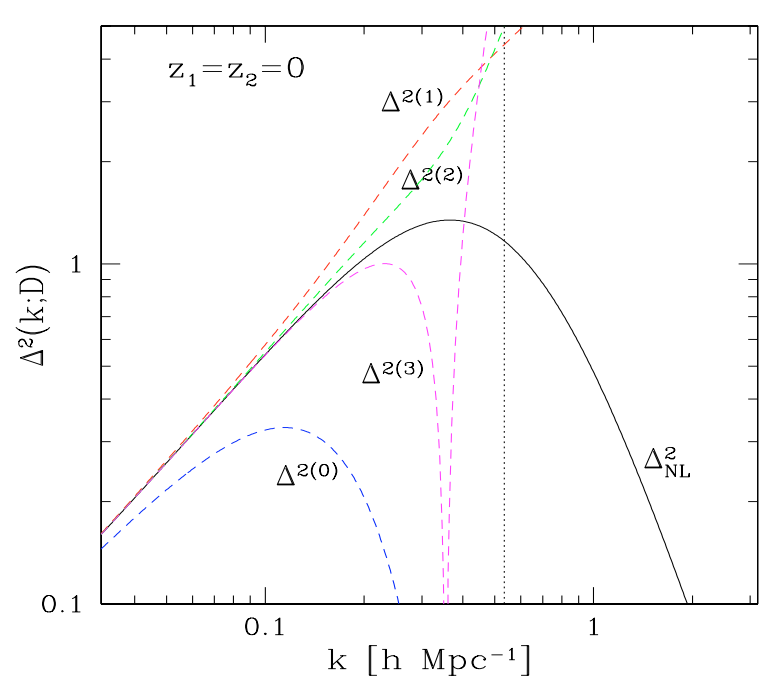

Fig. 10. The expansion of the two-point correlation defined by the direct steepest-descent method, from Eq. (179), but using the exact response function (133). Higher-order terms display a power-law growth at high $k$.

last term of Eq. (83). Indeed, expansion (179) yields power laws over $D_{1}, D_{2}$, which gives enough power generated at all times to build large density fluctuations into the nonlinear regime. We can see from Fig. 10 that using the exact response function is not enough to significantly improve the convergence of the series obtained for the matter power spectrum. Therefore, it appears that to improve the results one should use other expansion schemes for the self-energy $\hat{\Pi}$ : improving the response function alone does not help much.

\subsection{Using a Gaussian decay for the self-energy $\Pi$}

The previous discussion and the results obtained from expansion (159), shown in Fig. 5, suggest that it may be useful to factor out a Gaussian term of the form $\mathrm{e}^{-D^{2} \omega^{2}}$ from the self-energy $\Pi$. Therefore, we now replace expansion (179) by

$\hat{\Pi}\left(k ; D_{1}, D_{2}\right)=\frac{\mathrm{e}^{-\left(\omega_{1}^{2}+\omega_{2}^{2}\right) / 2}}{4 \pi k^{3}} \sum_{n=1}^{\infty} \hat{\Pi}_{n}\left(k ; D_{1}, D_{2}\right)\left(\begin{array}{cc}1 & 1 \\ 1 & 1\end{array}\right)$

where $\hat{\Pi}_{n}$ is again of order $P_{\mathrm{L} 0}^{n+1}$ and $\omega_{i}=D_{i} \omega$ as in Eq. (182). This expansion can be obtained as in Sect. 8.3 or it can be derived from the expansion (179) by multiplying by a factor $\mathrm{e}^{\left(\omega_{1}^{2}+\omega_{2}^{2}\right) / 2}$ and again expanding over powers of $P_{\mathrm{L} 0}$. We display in Fig. 11 the results obtained from Eq. (186) using the exact response function (133) that also shows a Gaussian decay at high $k$. Of course, because of the Gaussian damping factor introduced in Eq. (186) all terms decay as $\mathrm{e}^{-k^{2} \sigma_{v}^{2}}$ at high $k$ and the expansion looks better behaved than the one displayed in Fig. 10. Besides, the approximation obtained at a given order seems to provide good accuracy over a slightly wider range than in both Figs. 10 and 5. Thus, decomposing the two-point correlation in terms of response function and self-energy as in Eq. (83) and using a Gaussian decay ansatz at high $k$ appears to be a good scheme. However, this is somewhat artificial (see Sect. 9).

As explained in Sect. 9 below, the Gaussian damping factors $\mathrm{e}^{-D^{2} k^{2} \sigma_{v}^{2} / 2}$ merely correspond to the linear displacement field that moves the location of large-scale structures between

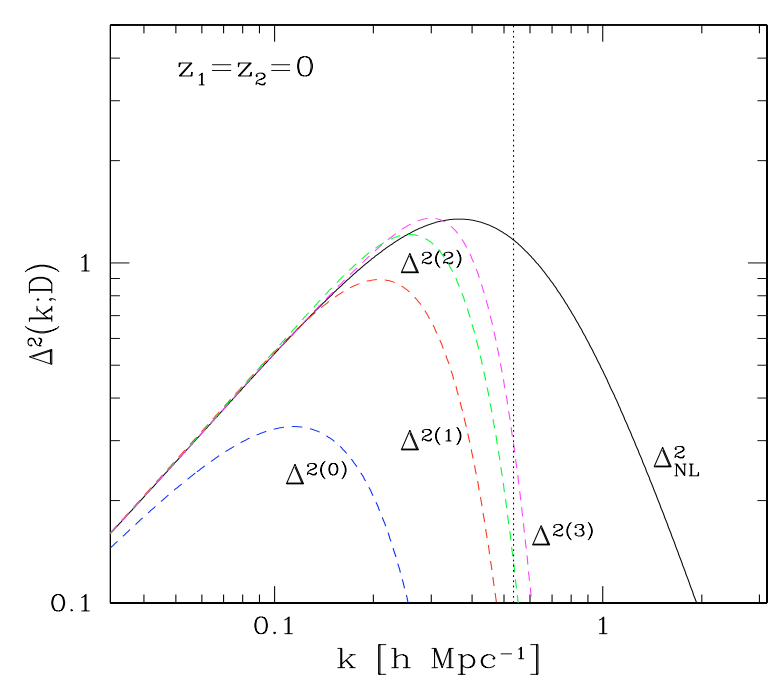

Fig. 11. The expansion of the two-point correlation from Eq. (186), using the exact response function (133) and the expansion (186) with Gaussian factors for the self-energy $\hat{\Pi}$. All terms display a Gaussian decay at high $k$.

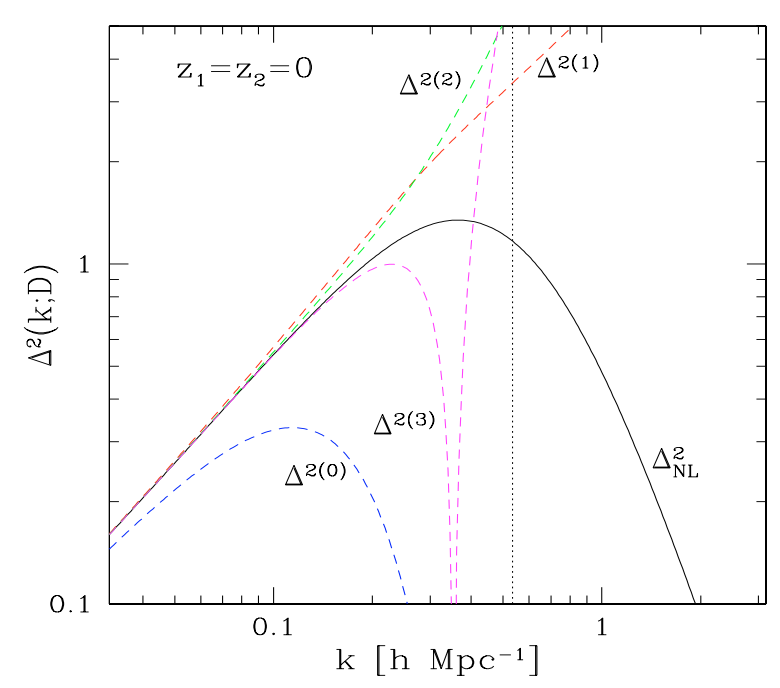

Fig. 12. The expansion of the two-point correlation from Eq. (187), using the exact response function (133) and the expansion (187) with Gaussian factors for the self-energy $\hat{\Pi}$. This Gaussian factor only damps the contributions at different times, and high-order terms display a power-law growth at high $k$.

different times. However, this process does not affect the matter clustering, and in particular the linear velocity variance $\sigma_{v}$ can show IR divergences for $n \leq-1$, which cancel out for equaltime statistics (Vishniac 1983; Jain \& Bertschinger 1996). This suggests that it would make more sense to factor out a Gaussian factor of the form

$\hat{\Pi}\left(k ; D_{1}, D_{2}\right)=\frac{\mathrm{e}^{-\left(\omega_{1}-\omega_{2}\right)^{2} / 2}}{4 \pi k^{3}} \sum_{n=1}^{\infty} \hat{\Pi}_{n}\left(k ; D_{1}, D_{2}\right)\left(\begin{array}{cc}1 & 1 \\ 1 & 1\end{array}\right)$,

which is equal to unity at equal times. This also agrees with the functional form of the exact two-point function (112) and of the simple approximation (199) below. We show the results of the expansion (187) in Fig. 12, again using the exact response 

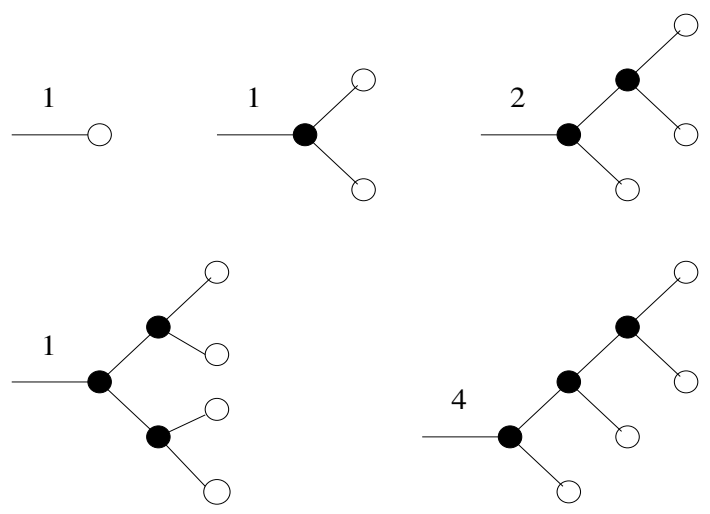

Fig. 13. The expansion of the nonlinear field $\psi$ over the linear growing mode $\psi_{\mathrm{L}}$ from Eq. (51), up to order $\psi_{\mathrm{L}}^{4}$. The filled circles are the vertex $\tilde{K}_{\mathrm{s}}$, whereas the white circles are the linear input $\psi_{\mathrm{L}}$. The numbers are the multiplicity factor associated with each diagram.

function (133). The high-order terms now grow as power laws at high $k$ for the equal-time power (clearly they would still decay as a Gaussian for unequal times), but the expansion does not fare better than the straightforward expansion obtained from Eq. (179) displayed in Fig. 10. This shows that using a reasonable Gaussian decay ansatz (which must disappear at equal times) is not sufficient to bring a significant improvement over previous expansions.

\section{High-k limit?}

To improve the behavior of expansion schemes, Crocce \& Scoccimarro (2006b) suggest using a response function that matches both the low- $t$ behavior (obtained by perturbative expansions) and the Gaussian decay at high $t$. Indeed, for the case of the gravitational dynamics where the exact response $R$ is not known, one can still obtain the low- $t$ behavior by perturbative expansions, such as those described above, by summing over higher-loop diagrams; see Crocce \& Scoccimarro (2006a) and Valageas (2007). On the other hand, Crocce \& Scoccimarro (2006b) manage to resum a subset of diagrams in the high- $k$ limit, which gives rise to the same Gaussian decay $\mathrm{e}^{-t^{2} / 2}$ as for the Zeldovich dynamics. Then, one may hope that by using a "fit" for the response function that closely follows the expected behavior, one has done half the work needed to obtain a good prescription for the matter power spectrum, and all that is left is to use a good recipe for the self-energy $\Pi$. In fact, we have shown in Sect. 8.3 and Fig. 10 that this is not so simple, because even using the exact response $R$ is not enough to improve the predictions for the power spectrum if we use a simple expansion over powers of $P_{\mathrm{L} 0}$ for the self-energy $\Pi$, as in Eq. (179). Moreover, as shown in Sect. 8.4 using such a Gaussian cutoff for $\Pi$ is not sufficient either.

Nevertheless, this section revisits the approximate resummation performed in Crocce \& Scoccimarro (2006b) to clarify its meaning and its shortcomings. Let us start from the general integral equation of motion (51). This equation can be solved perturbatively as an expansion over powers of $\psi_{\mathrm{L}}$ of the form

$\psi=\psi_{\mathrm{L}}+\tilde{K}_{\mathrm{s}} \psi_{\mathrm{L}}^{2}+2 \tilde{K}_{\mathrm{s}}^{2} \psi_{\mathrm{L}}^{3}+5 \tilde{K}_{\mathrm{s}}^{3} \psi_{\mathrm{L}}^{4}+\cdots$

which can also be written in a diagrammatic manner, see Crocce \& Scoccimarro (2006a) and Valageas (2001), as shown in Fig. 13. In the high- $k$ limit, one assumes that all wavenumbers $w_{i}$ associated with the linear fields $\psi_{\mathrm{L}}$ are much smaller
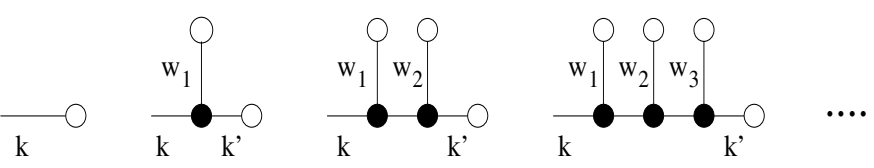

Fig. 14. The diagrams assumed to dominate in the high- $k$ limit. We have $k^{\prime} \simeq k$, and all intermediate wavenumbers $w_{i}$ are much smaller than $k$.

than $k$ except for one field (because of the conservation of momentum associated with the Dirac factor $\delta_{\mathrm{D}}\left(\boldsymbol{k}_{1}+\boldsymbol{k}_{2}-\boldsymbol{k}\right)$ in the vertex $K_{\mathrm{s}}$, see Eq. (20)). This assumes that the power is generated over some finite range of wavenumbers (i.e. the dynamics is not governed by an extended UV tail). Crocce \& Scoccimarro (2006b) assume that the dominant contribution is provided by the diagrams shown in Fig. 14 where all low- $w_{i}$ fields $\psi_{\mathrm{L}}\left(\boldsymbol{w}_{i}\right)$ are directly connected to the "principal path" that joins the only one high- $k$ field $\psi_{\mathrm{L}}$ to the root of the diagram. The idea is that in such diagrams the "principal path" only interacts with other fields $\psi_{\mathrm{L}}\left(\boldsymbol{w}_{i}\right)$, which are pure linear growing modes, as they have not already suffered nonlinear interactions through the coupling vertex $\tilde{K}_{\mathrm{s}}$. Then, these diagrams should maximize the crosscorrelations since nonlinear interactions are expected to erase the memory of initial conditions. Therefore, the response function $R$ may be dominated by these diagrams in the high- $k$ limit.

On the other hand, the diagrams of Fig. 14 are actually generated by the equation of motion

$\hat{\psi}(x)=\psi_{\mathrm{L}}(x)+2 \tilde{K}_{\mathrm{s}}\left(x ; x_{1}, x_{2}\right) \cdot \psi_{\mathrm{L}}^{<}\left(x_{1}\right) \hat{\psi}\left(x_{2}\right)$,

where $\psi_{\mathrm{L}}^{<}\left(x_{1}\right)$ is the linear growing mode restricted to low wavenumbers $w_{1} \ll k$ (the factor 2 comes from the fact that we can associate $\psi_{\mathrm{L}}^{<}$to either $x_{1}$ or $x_{2}$ in Eq. (51)), and we noted by a hat the approximate field $\hat{\psi}$ obtained with this high- $k$ limit. Note that $\psi_{\mathrm{L}}(x)$ and $\psi_{\mathrm{L}}^{<}\left(x_{1}\right)$ are independent Gaussian fields (since $\left.w_{1} \neq k\right)$ and Eq. (189) is now a linear equation for the field $\hat{\psi}$. Indeed, we can check that, by solving Eq. (189) as a perturbative series over powers of $\psi_{\mathrm{L}}$, we recover the diagrams of Fig. 14. In other words, by keeping only the diagrams of Fig. 14 we have actually approximated the nonlinear equation of motion (51) by the linear equation of motion (189). Next, following Crocce \& Scoccimarro (2006b) we note that the vertices $\gamma^{s}$ of Eqs. (21), (22) satisfy at the high- $k$ limit,

$$
\frac{k}{w} \gg 1: \sum_{m} \gamma_{i ; m j}^{s}(\boldsymbol{w}, \boldsymbol{k}) \psi_{\mathrm{L} m}^{<}\left(\boldsymbol{w}, \eta^{\prime}\right) \simeq \delta_{i, j} \frac{\boldsymbol{k} \cdot \boldsymbol{w}}{2 w^{2}} \mathrm{e}^{\eta^{\prime}} \delta_{\mathrm{L} 0}(\boldsymbol{w}),
$$

where we have only kept the terms of order $k / w$ and neglected terms of order $1, w / k, \ldots$ At this level, we can also replace the Dirac factor $\delta_{\mathrm{D}}\left(\boldsymbol{w}+\boldsymbol{k}^{\prime}-\boldsymbol{k}\right)$ by $\delta_{\mathrm{D}}\left(\boldsymbol{k}^{\prime}-\boldsymbol{k}\right)$ in the vertex $K_{\mathrm{s}}$, and using the second Eqs. (52) and (20), we can write Eq. (189) as

$$
\hat{\delta}(\boldsymbol{k}, \eta)=\mathrm{e}^{\eta} \delta_{\mathrm{L} 0}(\boldsymbol{k})+\int \mathrm{d} \boldsymbol{w} \frac{\boldsymbol{k} \cdot \boldsymbol{w}}{w^{2}} \delta_{\mathrm{L} 0}(\boldsymbol{w}) \mathrm{e}^{\eta} \int_{\eta_{\mathrm{I}}}^{\eta} \mathrm{d} \eta^{\prime} \hat{\delta}\left(\boldsymbol{k}, \eta^{\prime}\right),
$$

where we use the fact that $\psi_{\mathrm{L}}$ is also the linear growing mode. Here we consider the case where the initial conditions are set up at a finite time $\eta_{\mathrm{I}}$ as in Sect. 3.2.2. This linear equation can be solved through the expansion

$$
\begin{aligned}
\hat{\delta}(\boldsymbol{k}, \eta)= & \mathrm{e}^{\eta} \delta_{\mathrm{L} 0}(\boldsymbol{k})+\mathrm{e}^{\eta} \delta_{\mathrm{L} 0}(\boldsymbol{k}) \sum_{p=1}^{\infty} \prod_{j=1}^{p} \int \mathrm{d} \boldsymbol{w}_{j} \frac{\boldsymbol{k} \cdot \boldsymbol{w}_{j}}{w_{j}^{2}} \delta_{\mathrm{L} 0}\left(\boldsymbol{w}_{j}\right) \\
& \times \int_{\eta_{\mathrm{I}}}^{\eta} \mathrm{d} \eta_{1} \mathrm{e}^{\eta_{1}} \int_{\eta_{\mathrm{I}}}^{\eta_{1}} \mathrm{~d} \eta_{2} \mathrm{e}^{\eta_{2}} \cdot \int_{\eta_{\mathrm{I}}}^{\eta_{p-1}} \mathrm{~d} \eta_{p} \mathrm{e}^{\eta_{p}}
\end{aligned}
$$


which can be resummed as:

$$
\hat{\delta}(\boldsymbol{k}, D)=D \delta_{\mathrm{L} 0}(\boldsymbol{k}) \mathrm{e}^{\left(D-D_{\mathrm{I}}\right) \int \mathrm{d} \boldsymbol{w} \frac{\boldsymbol{k} \cdot \boldsymbol{w}}{w^{2}} \delta_{\mathrm{L} 0}(\boldsymbol{w})},
$$

where we use the time-coordinate $D=\mathrm{e}^{\eta}$. Let us recall that $\delta_{\mathrm{L} 0}(\boldsymbol{k})$ and $\delta_{\mathrm{L} 0}(\boldsymbol{w})$ must be treated as independent Gaussian variables in Eq. (193). Then, if we define a response $\hat{R}_{\mathrm{I}}\left(\boldsymbol{k}, \eta ; \boldsymbol{k}^{\prime}\right)$ in a fashion similar to Eq. (63) by

$\hat{R}_{\mathrm{I}}\left(\boldsymbol{k}, \eta ; \boldsymbol{k}^{\prime}\right)=\left\langle\frac{\mathcal{D} \hat{\delta}(\boldsymbol{k}, \eta)}{\mathcal{D} \delta_{\mathrm{LI}}\left(\boldsymbol{k}^{\prime}\right)}\right\rangle=\frac{1}{D_{\mathrm{I}}}\left\langle\frac{\mathcal{D} \hat{\delta}(\boldsymbol{k}, \eta)}{\mathcal{D} \delta_{\mathrm{L} 0}\left(\boldsymbol{k}^{\prime}\right)}\right\rangle$,

where $\mathcal{D}$ is the functional derivative, we obtain

$$
\begin{aligned}
\hat{R}_{\mathrm{I}} & =\delta_{\mathrm{D}}\left(\boldsymbol{k}-\boldsymbol{k}^{\prime}\right) \frac{D}{D_{\mathrm{I}}}\left\langle\mathrm{e}^{\left(D-D_{\mathrm{I}}\right) \int \mathrm{d} \boldsymbol{w} \frac{\boldsymbol{k} w}{w^{2}} \delta_{\mathrm{L} 0}(\boldsymbol{w})}\right\rangle \\
& =\delta_{\mathrm{D}}\left(\boldsymbol{k}-\boldsymbol{k}^{\prime}\right) \frac{D}{D_{\mathrm{I}}} \mathrm{e}^{-\frac{1}{2}\left(D-D_{\mathrm{I}}\right)^{2} k^{2} \sigma_{v}^{2}} .
\end{aligned}
$$

Thus we recover the exact Gaussian decay at high $k$ obtained by Crocce \& Scoccimarro (2006b). Note that since we have restricted the initial conditions to the linear growing mode in Eq. (191), the response $\hat{R}_{\mathrm{I}}$ of Eq. (194) actually corresponds to the sum $\tilde{\mathcal{R}}_{11}+\widetilde{\mathcal{R}}_{12}$ of the components of the response defined in Eq. (63). The advantage of this formulation is that one may see more clearly through Eqs. (189)-(193) the meaning of the assumptions involved in this high- $k$ limit. In particular, it is interesting to compare Eq. (193) with the exact result (98), which reads as

$\delta(\boldsymbol{k}, D)=\int \frac{\mathrm{d} \boldsymbol{q}}{(2 \pi)^{3}} \mathrm{e}^{-\mathrm{i} \boldsymbol{k} \cdot \boldsymbol{q}} \mathrm{e}^{D \int \mathrm{d} \boldsymbol{w} \mathrm{e}^{\mathrm{i} \boldsymbol{w} \cdot \boldsymbol{q}} \frac{\boldsymbol{k} \cdot \boldsymbol{w}}{w^{2}} \delta_{\mathrm{L} 0}(\boldsymbol{w})}$.

Then, if we assume that we can split the integral over $w$ into low and high wavenumber parts $w<\Lambda$ and $w>\Lambda$, such that most of the power is associated with $w<\Lambda$ and the high-wavenumber contribution is small, we can expand the exponential over this high-wavenumber part as

$$
\begin{aligned}
& \delta(\boldsymbol{k}, D)=\int \frac{\mathrm{d} \boldsymbol{q}}{(2 \pi)^{3}} \mathrm{e}^{-\mathrm{i} \boldsymbol{k} \cdot \boldsymbol{q}} \mathrm{e}^{D \int_{w<\Lambda} \mathrm{d} \boldsymbol{w} \mathrm{e}^{\mathrm{i} w \cdot \boldsymbol{q}} \frac{\boldsymbol{k} \cdot \boldsymbol{w}}{w^{2}} \delta_{\mathrm{L} 0}(\boldsymbol{w})} \\
& \times\left[1+D \int_{w^{\prime}>\Lambda} \mathrm{d} \boldsymbol{w}^{\prime} \mathrm{e}^{\mathrm{i} \boldsymbol{w}^{\prime} \cdot \boldsymbol{q}} \frac{\boldsymbol{k} \cdot \boldsymbol{w}^{\prime}}{w^{\prime 2}} \delta_{\mathrm{L} 0}\left(\boldsymbol{w}^{\prime}\right)+\ldots\right] .
\end{aligned}
$$

Next, if we assume that this expression is dominated by $q \sim 1 / k$, we can neglect the factor $\mathrm{e}^{\mathrm{i} \boldsymbol{i} \cdot \boldsymbol{q}}$ in the exponent in the high- $k$ limit $k \gg \Lambda$. Then the integration over $\boldsymbol{q}$ yields the Dirac factor $\delta_{\mathrm{D}}\left(\boldsymbol{w}^{\prime}-\boldsymbol{k}\right)$ to obtain at lowest order (the factor 1 does not contribute)

$$
\delta(\boldsymbol{k}, D) \simeq D \delta_{\mathrm{L} 0}(\boldsymbol{k}) \mathrm{e}^{D \int_{w<\Lambda} \mathrm{d} \boldsymbol{w} \frac{\boldsymbol{k} w}{w^{2}} \delta_{\mathrm{L} 0}(\boldsymbol{w})} .
$$

Thus we recover Eq. (193) with $D_{\text {I }} \rightarrow 0$ and letting $\Lambda \rightarrow \infty$. Of course, the assumptions involved in the derivation of Eq. (198) from Eq. (196) are identical to those involved in the derivation of Eq. (193). To check whether these assumptions are valid, we can compare the nonlinear power spectrum predicted by Eq. (193) with the exact power studied in Sect. 6.1. This gives (with $D_{\mathrm{I}}=0$ )

$\hat{\Delta}^{2}\left(k ; D_{1}, D_{2}\right)=\Delta_{\mathrm{L}}^{2} \mathrm{e}^{-\frac{1}{2}\left(D_{1}-D_{2}\right)^{2} k^{2} \sigma_{v}^{2}}$.

We again recover the exact Gaussian decay at high $k$ for unequal times, which corresponds to the exponential term in the exact expression (112); but for equal times, we merely get back the linear power $\Delta_{\mathrm{L}}^{2}$. In fact, the analysis of Sect. 6.2 shows that the assumptions underlying Eq. (198) are not valid. For a power-law linear power spectrum (114), the derivation leading to Eq. (117) shows that, in the highly nonlinear regime the nonlinear power at wavenumber $k$ is associated with scales $q \sim k^{-1+(n+3) /(n+1)}$ and with linear wavenumbers $w \sim k^{1-(n+3) /(n+1)}$. Thus, for $-3<n<$ -1 , where the system is well-defined, we find that the power at a nonlinear wavenumber $k$ is generated by linear wavenumbers $w$, which actually grow faster ${ }^{2}$ than $k$ instead of being restricted to a finite range $w<\Lambda$. Therefore, one cannot define a fixed cutoff $\Lambda$ beyond which nonlinear interactions are negligible so that we can expand the high- $w$ part as in Eq. (197). The latter integral is actually large in the nonlinear regime, and one needs to take its full non-perturbative expression into account to obtain the nontrivial scaling of Eq. (118).

As was already clear from the linear Eqs. (189), (191), the high- $k$ approximations described above neglect the nonlinear interactions associated with high wavenumbers, which actually govern the formation of large-scale structures on small scales, and only keep track of the overall displacement associated with the large-scale velocity field. This is why the low- $k$ nonlinear interactions could be resummed as $\mathrm{e}^{-\left(D_{1}-D_{2}\right) k^{2} \sigma_{v}^{2}}$, which only involves the variance $\sigma_{v}$ of the linear displacement field. This is clearly an important feature of the dynamics for unequal times, especially for the simple Zeldovich dynamics studied in this paper where the exact trajectories satisfy the simple law (10) and the response function (133) happens to be fully determined by a similar advection process. In fact, Eq. (193) reads in real space (with $D_{\text {I }}=0$ ) as

$\hat{\delta}(\boldsymbol{x}, D)=\delta_{\mathrm{L}}\left(\boldsymbol{x}-\boldsymbol{s}_{\mathrm{L}}(\boldsymbol{q}=0, D)\right)$,

where $s_{\mathrm{L}}=D_{+} s_{\mathrm{L} 0}$ is the linear displacement field of Eq. (10). As expected, Eq. (200) explicitly shows that the approximation $\delta_{\mathrm{D}}\left(\boldsymbol{w}+\boldsymbol{k}^{\prime}-\boldsymbol{k}\right) \simeq \delta_{\mathrm{D}}\left(\boldsymbol{k}^{\prime}-\boldsymbol{k}\right)$ in the vertex $K_{\mathrm{s}}$ used to obtain Eq. (191) and to simplify the diagrams of Fig. 14 has broken the invariance through translations of the system. As seen above, this invariance is restored as in Eqs. (195) and (199) by treating $s_{\mathrm{L}}(\boldsymbol{q}=0)$ as an independent Gaussian random variable. Equation (200) clearly shows that the effective dynamics associated with these high- $k$ approximations is simply the uniform advection of the linear density field by the linear velocity at $\boldsymbol{q}=0$. After averaging over the Gaussian initial conditions, this random displacement of large-scale structures leads to an apparent "diffusion" in the form of a Gaussian decay $\mathrm{e}^{-k^{2} \sigma_{v}^{2}}$ for both the response function and the different-time correlation, see Eqs. (195) and (199). This apparent loss of memory happens to dominate the different-time behavior of two-point functions for the Zeldovich dynamics, as seen from Eqs. (112) and (133), but

2 That the power at a nonlinear wavenumber $k$ mostly comes from higher linear wavenumbers $w$, as shown by the explicit expression (109) analyzed in Sect. 6.2, may seem a bit counter-intuitive as one may expect a "direct cascade" from larger to smaller scales. However, it appears that the actual process is somewhat more complicated within the highly nonlinear regime. In particular, as analyzed in Taylor \& Hamilton (1996) (see also Schneider \& Bartelmann 1995), the equaltime nonlinear power behaves as $\Delta^{2}(k, D) \propto D^{-3}$ (which is independent of $k$ ) in the highly nonlinear regime if the spectral index is less than -3 on small scales (e.g. the linear power shows a high- $k$ cutoff $\left.P_{\mathrm{L}}(k) \propto k^{n} \mathrm{e}^{-k^{2} / \Lambda^{2}}\right)$. This scaling can be read from Eq. (105) with $w$ fixed and $q \sim 1 /(D k)$, which shows that the power comes from a fixed range of wavenumbers $w$ (e.g. $w<\Lambda$ ) associated with caustics (Schneider $\&$ Bartelmann 1995). However, for a spectral index larger than -3 on small scales the scaling is quite different as shown by Eq. (118) (see also Taylor \& Hamilton 1996), and one cannot neglect smaller scales to describe the nonlinear evolution. 
it is actually disconnected from the building of matter clustering as is obvious from Eq. (200).

It is clear that all steps going from Eqs. (188) to (195), and Eqs. (199)-(200) can be applied identically to the exact gravitational dynamics, see Crocce \& Scoccimarro (2006b). As seen above, the high- $k$ approximations associated with Eq. (193) cannot aim at capturing the physics of gravitational clustering but only the "diffusion" associated with the linear velocity variance, which affects different-time statistics. However, it is not obvious that the approximate response (195) should again agree with the exact response in the high- $k$ limit, which may thus depart from such a Gaussian decay. The small-scale gravitational dynamics is indeed quite different from the simple Zeldovich dynamics and other processes may come into play. Nevertheless, the apparent loss of memory due to the almost uniform random displacement of large-scale structures by low- $k$ modes, which is captured by the simple dynamics (200), clearly applies to the exact gravitational dynamics as well. Therefore, one can expect again a decay as fast as in Eq. (195) although only wavelengths which are still linear would contribute to $\sigma_{v}$ (it is not clear whether nonlinear wavelengths would give rise to a stronger or weaker decay as compared with Eq. (195)). On the other hand, for the exact gravitational dynamics the fluid equations break down beyond shell-crossing so that the small-scale limit associated with these hydrodynamical equations is not so well defined.

\section{2PI effective action method}

We now investigate the 2PI effective action method presented in Sect. 4.2 and described in detail in Valageas (2007). Thus, we need to solve the system of coupled Eqs. (70)-(72) and (81), (82). Thanks to causality, which leads to the Heaviside factor $\theta\left(\eta_{1}-\eta_{2}\right)$ within both $R$ and $\Sigma$, we solve this system by moving forward over time. We refer the reader to Valageas (2007) for a description of the numerical scheme. We consider the $\Lambda \mathrm{CDM}$ cosmology associated with Eq. (153) and we only investigate the one-loop predictions.

We first display in Fig. 15 the evolution forward over time $D_{1}$ of the response $R\left(k ; D_{1}, D_{2}\right)$. We can see that the nonlinear response exhibits oscillations as for the steepest-descent result (163) but its amplitude now decays as an inverse powerlaw at large times $D_{1}$ instead of following the linear envelope (at one-loop order). As shown in Sect. 6.1 of Valageas (2007) this behavior is due to the nonlinearity of the Schwinger-Dyson equation for the response $R$. Of course, in the weakly nonlinear regime we also recover the exact response (133) (dashed lines). Next, we display in Fig. 16 the response function as a function of wavenumber $k$. In agreement with Fig. 15, we again obtain damped oscillations in the nonlinear regime. This is a clear improvement over both the standard perturbative expansion displayed in Fig. 3 and the steepest-descent result displayed in Fig. 6. This behavior is identical to the one obtained for the gravitational dynamics studied in Valageas (2007).

Finally, we show the logarithmic power $\Delta^{2}\left(k ; D_{1}, D_{2}\right)$ as a function of wavenumber $k$ in Figs. 17 and 18. We compare the 2PI effective action prediction at one-loop order with the linear power, the exact nonlinear power obtained from Eq. (109), and the usual one-loop result obtained from standard perturbative analysis. The latter may also be obtained by expanding Eq. (109) up to order $P_{\mathrm{L} 0}^{2}$, and it reads for the Zeldovich dynamics as,

$P^{1 \text { loop }}\left(k ; D_{1}, D_{2}\right)=P_{\mathrm{L}}+P_{22}+P_{13}$,

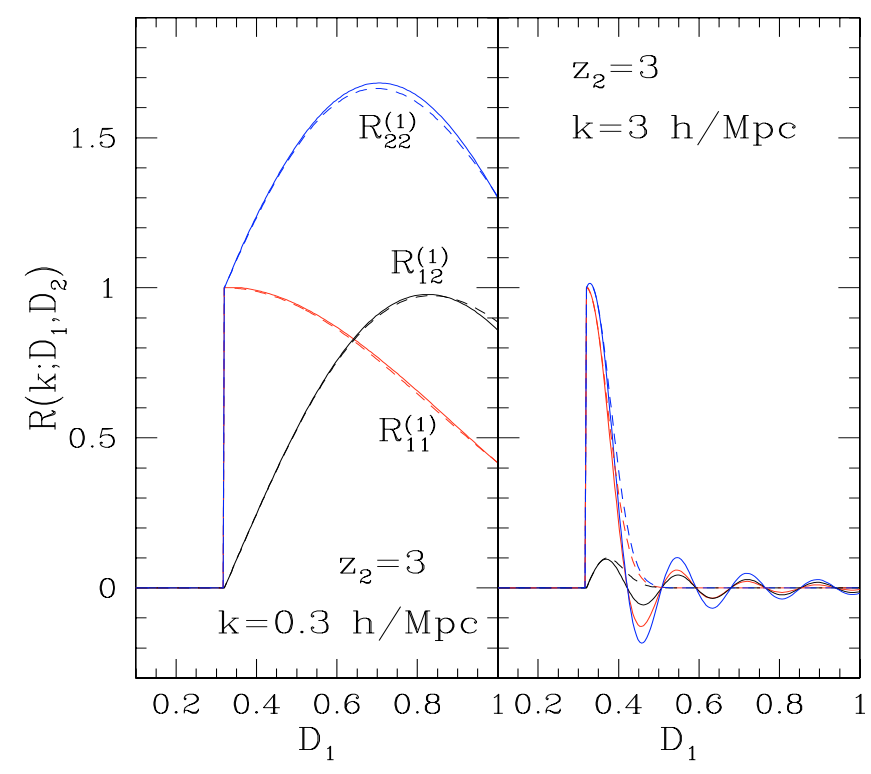

Fig. 15. The nonlinear response $R\left(k ; D_{1}, D_{2}\right)$ (solid lines) as a function of forward time $D_{1}$, for $D_{2}=0.32$ (i.e. $z_{2}=3$ ) and wavenumbers $k=0.3$ (left panel) and $3 \times h \mathrm{Mpc}^{-1}$ (right panel) for the 2PI effective action method at one-loop order. For comparison we also plot the exact response $R_{\mathrm{NL}}$ (dashed lines).

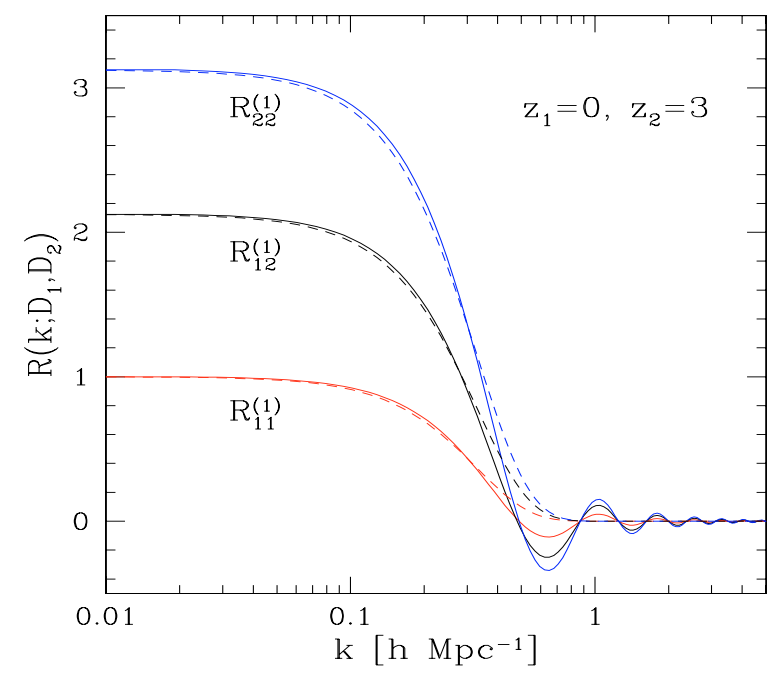

Fig. 16. The nonlinear response function $R\left(k ; D_{1}, D_{2}\right)$ (solid lines) as a function of wavenumber $k$, at times $z_{1}=0, z_{2}=3$, for the 2PI effective action method at one-loop order. We also plot the exact nonlinear response $R_{\mathrm{NL}}$ (dashed lines).

with

$P_{\mathrm{L}}=D_{1} D_{2} P_{\mathrm{L} 0}(k)$

$P_{13}=-\frac{D_{1}^{2}+D_{2}^{2}}{2} D_{1} D_{2} P_{\mathrm{L} 0}(k) k^{2} \sigma_{v}^{2}$

$P_{22}=D_{1}^{2} D_{2}^{2} \int \mathrm{d} \boldsymbol{w} \frac{(\boldsymbol{k} \cdot \boldsymbol{w})^{2}[\boldsymbol{k} \cdot(\boldsymbol{k}-\boldsymbol{w})]^{2}}{2 w^{4}|\boldsymbol{k}-\boldsymbol{w}|^{4}} P_{\mathrm{L} 0}(w) P_{\mathrm{L} 0}(|\boldsymbol{k}-\boldsymbol{w}|)$

The results match the steepest-descent predictions, as well as the usual one-loop power (201), on large scales. On small scales, 


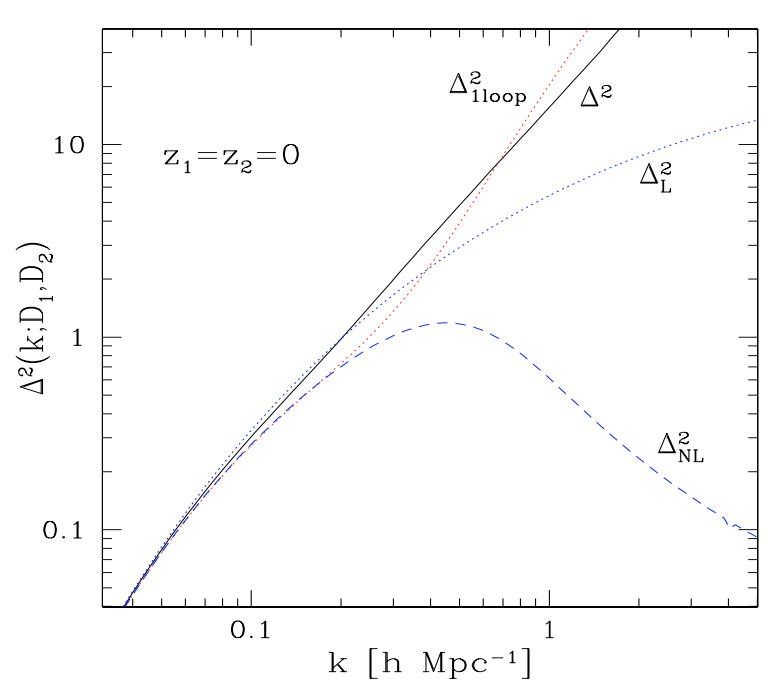

Fig. 17. The logarithmic power $\Delta^{2}(k)$ (solid line) at redshift $z=0$, that is, at equal times $z_{1}=z_{2}=0$. We also display the linear power $\Delta_{\mathrm{L}}^{2}$ (dotted line), the usual one-loop perturbative result $\Delta_{\text {lloop }}^{2}$ of Eq. (201) (dotted line) and the exact nonlinear power $\Delta_{\mathrm{NL}}^{2}$ of Eq. (109) (dashed line).

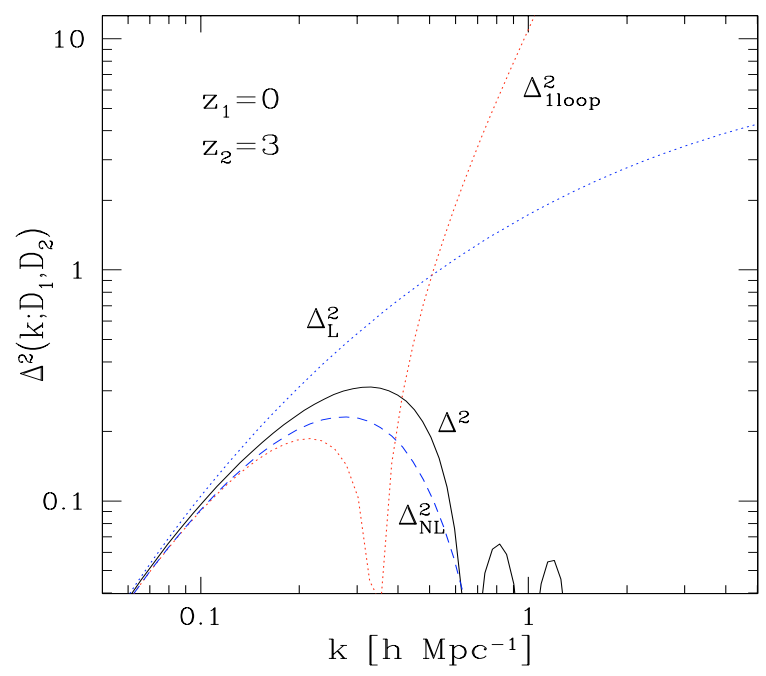

Fig. 18. The logarithmic power $\Delta^{2}(k)$ at unequal times $\left(D_{1}=1, D_{2}=\right.$ 0.32 ) (i.e. $z_{1}=0, z_{2}=3$ ).

contrary to the usual one-loop power (201) and the steepestdescent predictions, the 2PI effective action methods yields a logarithmic power $\Delta^{2}\left(k ; D_{1}, D_{2}\right)$, which decays for different times $D_{1} \neq D_{2}$, see Fig. 18. This high- $k$ power-law damping is due to the decay of the response function already shown in Figs. 15 and 16, leading to a decorrelation on small scales and for large time separations; however, it is only a power-law decay instead of the exact Gaussian damping seen in Eq. (112). On the other hand, for equal times $D_{1}=D_{2}=D$, we obtain a steady growth of the power $\Delta^{2}(k)$ in between the linear prediction $\Delta_{\mathrm{L}}^{2}$ and the usual one-loop prediction $\Delta_{\text {lloop }}^{2}$, see Fig. 17. This is due to the contributions of nearby times $D_{1}^{\prime} \simeq D_{2}^{\prime} \simeq D$ in the last term of Eq. (83), which are not damped because of their small time-difference. Note that the cancellation at equal times of the damping associated with the decay of the response function is qualitatively correct, as shown from the exact nonlinear solution studied in Sects. 6.1 and 6.2, see Eq. (112). Therefore, at oneloop order, the 2PI effective action method shows a significant qualitative improvement over the standard perturbative expansions of Sect. 7 and the direct steepest-descent method. However, it does not manage to predict the high- $k$ smooth power-law decay of the equal-time power $\Delta^{2}(k)$.

\section{Simple nonlinear schemes associated with the 2PI effective action method}

\subsection{Response function}

For the 2PI effective action method, it is not straightforward to obtain the self-energy $\Sigma$ at a given order from the exact expressions (140) or (142). Indeed, for the steepest-descent approach investigated in Sect. 8, the self-energy at a given order simply corresponds to the truncation of its expansion over powers of $P_{\mathrm{L} 0}$; therefore, it could be directly obtained by expanding the exact result. By contrast, within the 2PI effective action scheme, the self-energy is obtained from a diagrammatic expansion in terms of the nonlinear response $R$ and correlation $G$ (defined self-consistently at this order). Then, the exact expressions of the response $R$ and correlation $G$ are not sufficient to fully define the equations associated with the 2PI effective action method at any order, so one must go back to its diagrammatic definition. To avoid this complication, and to take advantage of the known expressions of the exact two-point functions, which allowed us to bypass the computation of high-order diagrams in Sect. 8, we investigate here a nonlinear expansion that is not identical to the 2PI effective action but is expected to show a similar behavior. Thus, we look for an expansion of the self-energy $\Sigma$ over the nonlinear response $R$. A simple way to build such an expansion is to use the expansions over $1 / s$ of the Laplace transforms $\tilde{r}(s)$ and $\tilde{\sigma}(s)$. Thus, from Eq. (164) and the Laplace transform of Eq. (142), we have

$$
\begin{aligned}
& \tilde{r}(s)=\frac{1}{s}-\frac{1}{s^{3}}+\frac{3}{s^{5}}-\frac{15}{s^{7}}+\frac{105}{s^{9}}+\cdots \\
& \tilde{\sigma}(s)=\frac{1}{s}-\frac{2}{s^{3}}+\frac{10}{s^{5}}-\frac{74}{s^{7}}+\frac{706}{s^{9}}+\cdots
\end{aligned}
$$

Then, the series (205) may be inverted as

$\frac{1}{s}=\tilde{r}+\tilde{r}^{3}+3 \tilde{r}^{7}-20 \tilde{r}^{9}+\cdots$

Composing this expansion with Eq. (206), we obtain

$\tilde{\sigma}=\tilde{r}-\tilde{r}^{3}+4 \tilde{r}^{5}-27 \tilde{r}^{7}+248 \tilde{r}^{9}+\cdots$

This provides an expansion of the self-energy $\Sigma$ in terms of the nonlinear response $R$. In real $t$-space this yields multiple integrals over $r(t)$

$\sigma(t)=r(t)-\int_{0}^{t} \mathrm{~d} t_{1} \int_{0}^{t_{1}} \mathrm{~d} t_{2} r\left(t-t_{1}\right) r\left(t_{1}-t_{2}\right) r\left(t_{2}\right)+\cdots$

in a fashion similar to what would be obtained for the diagrammatic expansion associated with the 2PI effective action. The main difference is that the expansion (208) only involves the response $R$, whereas the 2PI effective action expansion involves both $R$ and $G$, as in Eq. (81). Note that this shows that one can define several nonlinear expansion schemes. That it is possible to write a simple expansion such as (208) is due to the exact response $R$ only depending on the linear power spectrum through 
the velocity dispersion $\sigma_{v}^{2}$. This is not the case for the gravitational dynamics where it may not be possible to write an expansion for $\Sigma$ only in terms of $R$. Next, truncating the expansion (208) at a given order and substituting it into Eq. (136), we obtain at order $p=1$ :

$\tilde{\sigma}=\tilde{r}, \quad \tilde{r}^{2}+s \tilde{r}-1=0, \quad \tilde{r}^{(1)}(s)=\frac{\sqrt{s^{2}+4}-s}{2}$.

The root of the polynomial of degree $2 p$ which must be chosen, is the one that is consistent with the expansion (205) for $s \rightarrow$ $\infty$. Equation (210) is a well-known Laplace transform, and we obtain

$r^{(1)}(t)=\frac{J_{1}(2 t)}{t}$.

Note that this expression is also obtained as a simple approximation for the one-loop 2PI effective action approach for the gravitational dynamics in Valageas (2007). We also recover the damped oscillations obtained within the 2PI effective action method displayed in Figs. 15 and 16. Thus, as expected this nonlinear expansion and the 2PI effective action expansion show the same behavior at order $p=1$. At orders $p=2,3$, we obtain the polynomial equations:

$p=2:-\tilde{r}^{4}+\tilde{r}^{2}+s \tilde{r}-1=0$

$p=3: 4 \tilde{r}^{6}-\tilde{r}^{4}+\tilde{r}^{2}+s \tilde{r}-1=0$.

However, we now find that the solutions $\tilde{r}^{(p)}(s)$ defined from these equations have singularities in the right-hand half-plane $\operatorname{Re}(s)>0$. For order $p=2$, this may be directly seen from the explicit solution of Eq. (212) or from the behavior of the four roots $\tilde{r}_{i}(s)$ as a function of $s$ shown in left panel of Fig. 19. Indeed, as we follow $s$ along the real axis from $+\infty$ to 0 , the root of interest $\tilde{r}_{1}(s)$ that starts from $\tilde{r}_{1}=0$ at $s=+\infty$ "collides" with a second root $\tilde{r}_{2}$ at $\tilde{r}_{*} \simeq 0.87\left(s_{*} \simeq 0.94\right)$ and afterwards forms a pair of complex conjugates with $\tilde{r}_{2}$. This is associated with a square-root singularity for $\tilde{r}(s)$. (A simple example is provided by the polynomial $\tilde{r}^{2}-s=0$ with a singularity at $\tilde{r}_{*}=0, s_{*}=0$.) For order $p=3$, the right panel of Fig. 19, where we follow $s$ along the line $\operatorname{Im}(s)=1.052$ from $\operatorname{Re}(s)=+\infty$ down to $\operatorname{Re}(s)=0$, shows that we again have a singularity at the complex points $s_{*} \simeq 1.17 \pm 1.05 i, \tilde{r}_{*} \simeq 0.56 \mp 0.31 i$. These singularities yield exponential factors $\mathrm{e}^{s_{*} t}$ for the response $r(t)$, which grow at large $t$. Therefore, although the expansion (208) is much better than the steepest-descent approach (163) at order $p=1$, since it exhibits a damping in the nonlinear regime, it shows as for expansion (163) growing exponentials at higher orders. Thus, in this sense this nonlinear expansion is not wellbehaved. We can expect that a similar problem occurs for the 2PI effective action approach at high orders.

\subsection{Correlation function}

We now investigate nonlinear schemes for the two-point correlation $G$. As for the response function, we look for a simple nonlinear expansion that bypasses the need to compute high-order diagrams but that follows the structure of the 2PI effective action method. This is not as straightforward as for the response $R$ because the two-point correlation $G$ cannot be written in terms of a one-dimensional function such as $r(t)$ for the response $R$. Thus, we focus on the equal-time nonlinear power for the case of a power-law linear power spectrum and we write

$\Delta^{2}(k ; D)=\Delta_{\mathrm{L}}^{2} g(t)$ with $t=D \sqrt{\Delta_{\mathrm{L} 0}^{2}}=\sqrt{\Delta_{\mathrm{L}}^{2}}$,
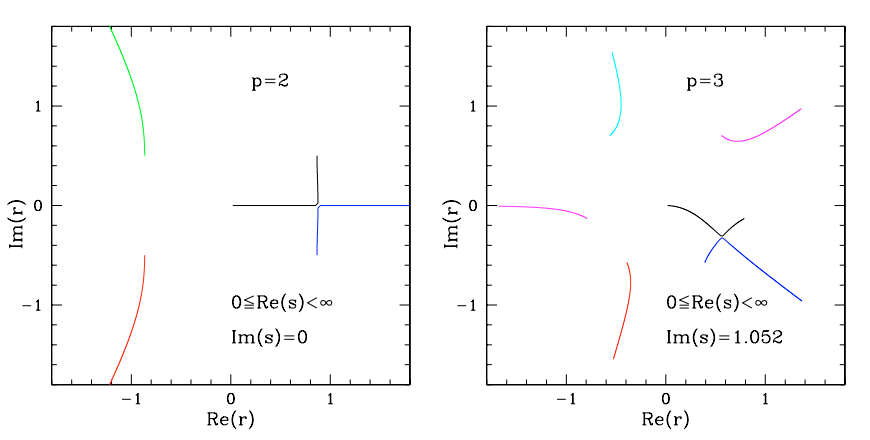

Fig. 19. Left panel: the trajectories of the four roots of Eq. (212) as we follow $s$ along the real axis from $s=+\infty$ down to $s=0$. Right panel: the trajectories of the six roots of Eq. (213) as we follow $s$ along the line $\operatorname{Im}(s)=1.052$ from $\operatorname{Re}(s)=+\infty$ down to $\operatorname{Re}(s)=0$. The root of interest starting from $\tilde{r}=0$ "collides" with a second root and changes direction by $\pi / 2$. This corresponds to a singularity for the implicit function $\tilde{r}(s)$.

which defines the time-variable $t$ used in this section and the function $g(t)$. From Eqs. (156) and (175), we have for $n=-2$

$g(t)=\sum_{p=1}^{\infty} \frac{F_{p}}{p !} t^{2 p-2}$

which yields for the Laplace transform defined as in Eq. (137):

$\tilde{g}(s)=\sum_{p=1}^{\infty} \frac{F_{p}}{p !}(2 p-2) ! s^{-2 p+1}=\frac{1}{s}+\frac{3 \pi^{2}}{32 s^{3}}-\frac{3 \pi^{2}}{4 s^{5}}+\cdots$

This series can be inverted as:

$\frac{1}{s}=\tilde{g}-\frac{3 \pi^{2}}{32} \tilde{g}^{3}+\frac{3\left(256 \pi^{2}+9 \pi^{4}\right)}{1024} \tilde{g}^{5}+\cdots$

Next, the derivative of $g(t)$ verifies

$g^{\prime}(t)=\sum_{p=2}^{\infty} \frac{F_{p}}{p !}(2 p-2) t^{2 p-3}$

and the Laplace transform of this equation reads

$$
\begin{aligned}
s \tilde{g}(s)-1 & =\sum_{p=2}^{\infty} \frac{F_{p}}{p !}(2 p-2) ! s^{-2 p+2} \\
& =\frac{3 \pi^{2}}{32 s^{2}}-\frac{3 \pi^{2}}{4 s^{4}}-\frac{675 \pi^{4}}{512 s^{6}}+\cdots,
\end{aligned}
$$

which could also be obtained from Eq. (216). Then, substituting the series (217) into Eq. (219) gives

$s \tilde{g}(s)-1=\frac{3 \pi^{2}}{32} \tilde{g}^{2}-\frac{3 \pi^{2}\left(128+3 \pi^{2}\right)}{512} \tilde{g}^{4}+\cdots$

Thus, as for the response function studied in Sect. 11.1, we have obtained a simple nonlinear expansion scheme for the two-point correlation $G$ (i.e. for the nonlinear power $\left.\Delta^{2}(k ; D)\right)$. However, since the functional dependence of the two-point correlation $G$ is not as simple as for response $R$, the expansion (220) only applies to the equal-time power, and it is not built from the selfenergy П. Nevertheless, going back to real $t$-space, Eq. (220) yields an integro-differential equation for $G$ such as Eq. (70), with a fixed differential term on the left hand side and a series of multiple integrals over $g(t)$ on the right hand side. At order 
$p=0$, the right hand side is zero and we recover the linear solution $g^{(0)}(t)=1$. At order $p=1$, we obtain

$s \tilde{g}-1=\frac{3 \pi^{2}}{32} \tilde{g}^{2}, \quad \tilde{g}^{(1)}(s)=\frac{16}{3 \pi^{2}}\left[s-\sqrt{s^{2}-3 \pi^{2} / 8}\right]$,

which gives

$g^{(1)}(t)=\sqrt{\frac{2}{3}} \frac{4}{\pi t} I_{1}\left(\sqrt{\frac{3}{2}} \frac{\pi t}{2}\right)$,

where $I_{1}$ is the modified Bessel function of order 1 . Thus, the nonlinear power $\Delta^{2(1)}$ shows an exponential growth in the highly nonlinear regime. We can check that at order $p=2$ we again have an exponential growth (with oscillations since $\operatorname{Im}\left(s_{*}\right) \neq 0$ where $s_{*}$ is the location of the singularity of $\left.\tilde{g}^{(2)}(s)\right)$. Therefore, the nonlinear expansion (220) is not better behaved than the "linear" expansions associated with the steepest-descent approach studied in Sect. 8.3.

\section{Simple nonlinear schemes associated with the running with the high-k cutoff}

\subsection{Response function}

In a fashion similar to Sect. 11, we now investigate some nonlinear schemes that may be built in the spirit of the method outlined in Sect. 5, where we considered the dependence of the system on a high- $k$ cutoff $\Lambda$. In order to separate the dependence on $\Lambda$, we now write the response function as

$R=R_{\mathrm{L}} r\left(t, \omega^{2}\right) \quad$ with $\quad t=D_{1}-D_{2}$,

$\omega^{2}=k^{2} \frac{4 \pi}{3} \int_{0}^{\Lambda} \mathrm{d} w P_{\mathrm{L} 0}(w)$.

Thus, the exact response function $r\left(t, \omega^{2}\right)$ is, from Eq. (133),

$r\left(t, \omega^{2}\right)=\mathrm{e}^{-\omega^{2} t^{2} / 2}$.

Defining the Laplace transform with respect to $t$ as in Eq. (137), we obtain

$\tilde{r}\left(s, \omega^{2}\right)=\sum_{p=0}^{\infty}(-1)^{p}(2 p-1) ! ! \omega^{2 p} s^{-2 p-1}$,

while the derivative with respect to $\Lambda$ is

$\frac{\partial \tilde{r}}{\partial \Lambda}\left(s, \omega^{2}\right)=\frac{\mathrm{d} \omega^{2}}{\mathrm{~d} \Lambda} \sum_{p=1}^{\infty}(-1)^{p}(2 p-1) ! ! p \omega^{2(p-1)} s^{-2 p-1}$.

Inverting the series (226) and substituting into Eq. (227) gives the expansion

$\frac{\partial \tilde{r}}{\partial \Lambda}=\frac{\mathrm{d} \omega^{2}}{\mathrm{~d} \Lambda}\left[-\tilde{r}^{3}+3 \omega^{2} \tilde{r}^{5}-18 \omega^{4} \tilde{r}^{7}+\cdots\right]$.

At order $p=0$, we have $\partial \tilde{r} / \partial \Lambda=0$ and we recover the linear response (since we impose $\left.r(\Lambda=0)=r_{\mathrm{L}}\right)$. At order $p=1$ we obtain

$\frac{\partial \tilde{r}}{\partial \Lambda}=-\frac{\mathrm{d} \omega^{2}}{\mathrm{~d} \Lambda} \tilde{r}^{3}$

which is similar to Eq. (91) once we go back to real $t$-space, which leads to a double integral over time (as in Eq. (209)). We can recover Eq. (92) by noting that the linear response is
$\tilde{r}_{\mathrm{L}}(s)=1 / s$, whence $\tilde{r}_{\mathrm{L}}^{3}=1 / 2 \mathrm{~d}^{2} \tilde{r}_{\mathrm{L}} / \mathrm{d} s^{2}$. Substituting this result into Eq. (229) gives $\partial \tilde{r} / \partial \Lambda=-1 / 2\left(\mathrm{~d} \omega^{2} / \mathrm{d} \Lambda\right) \mathrm{d}^{2} \tilde{r}_{\mathrm{L}} / \mathrm{d} s^{2}$. The inverse Laplace transform of this equation gives back Eq. (92). It is clear that this procedure is not systematic and only applies to the linear response. This is why we could reduce the three response functions on the right hand side of Eq. (91) to the one response function on the right hand side of Eq. (92). Then, replacing $R_{\mathrm{L}}$ by $R$ in Eq. (92) is clearly correct at the order $\omega^{2}$ at which the right hand side of Eq. (90) was truncated, but this method cannot be extended to higher orders in a systematic fashion. Thus, let us consider Eq. (229) with keeping the right hand side as it comes out from the systematic expansion obtained in (228). Integrating over $\Lambda$ gives

$\tilde{r}^{(1)}(s)=\frac{1}{\sqrt{s^{2}+2 \omega^{2}}}$ whence $r^{(1)}(t)=J_{0}(\sqrt{2} \omega t)$.

Thus, we obtain decaying oscillations into the nonlinear regime, which are damped as $\left[k\left(D_{1}-D_{2}\right)\right]^{-1 / 2}$. Note that the damping is smaller than for the 2PI effective action method where the simplified expansion (208) gave Eq. (211), which decays as $\left[k\left(D_{1}-D_{2}\right)\right]^{-3 / 2}$. At next order $p=2$ we obtain

$$
\frac{\partial \tilde{r}}{\partial \Lambda}=\frac{\mathrm{d} \omega^{2}}{\mathrm{~d} \Lambda}\left[-\tilde{r}^{3}+3 \omega^{2} \tilde{r}^{5}\right] \text { hence } \frac{\partial \tilde{r}}{\partial \omega^{2}}=-\tilde{r}^{3}+3 \omega^{2} \tilde{r}^{5}
$$

Then, looking for a solution of the form

$r\left(t, \omega^{2}\right)=\rho(\omega t), \quad \tilde{r}\left(s, \omega^{2}\right)=\frac{1}{\omega} \tilde{\rho}(y)$ with $y=\frac{s}{\omega}$,

we obtain for the Laplace transform $\tilde{\rho}(y)$ the equation

$\tilde{\rho}+y \tilde{\rho}^{\prime}=2 \tilde{\rho}^{3}-6 \tilde{\rho}^{5}$.

Note that Eq. (233) no longer involves an explicit dependence on $\omega$. It can be solved in implicit form as

$y=\frac{1}{\tilde{\rho}}\left(1-2 \tilde{\rho}^{2}+6 \tilde{\rho}^{4}\right)^{1 / 4} \mathrm{e}^{\left[\arctan \sqrt{5}-\arctan \left(\frac{\sqrt{5}}{1-6 \tilde{\rho}^{2}}\right)\right] /(2 \sqrt{5})}$.

Going back to $s=\omega y$, we see that $\tilde{r}\left(s, \omega^{2}\right)$ is singular at the point

$s_{*}=\omega y_{*} \quad$ with $\quad y_{*}=6^{1 / 4} \mathrm{e}^{\frac{1}{2 \sqrt{5}} \arctan \sqrt{5}}, \quad s_{*}>0$,

where $|\tilde{r}|=\infty$. Therefore, the response function obtained at order $p=2$ grows into the nonlinear regime as $r^{(2)} \sim \mathrm{e}^{s_{*} t}$, which gives $R^{(2)} \sim \mathrm{e}^{y_{*} k \sigma_{v}\left(D_{1}-D_{2}\right)}$. Thus, as for the nonlinear scheme of Sect. 11.1 associated with the 2PI effective action method, we find that at high orders, the expansion (228) gives rise to response functions that exhibit an exponential growth into the nonlinear regime, even though at lowest-order $p=1$, it managed to provide a nonlinear damping. Therefore, that the Gaussian decay could be recovered from Eq. (92) is not due to the good convergence properties of the method outlined in Sect. 5. As discussed in Sect. 5, from Eq. (94) it merely comes from the successive steps that have been performed using the properties of the linear response until one gets the linear Eq. (92), which is correct at lowest order and which has the desired solution. However, these intermediate steps cannot be directly extended to high orders without ambiguities, and the systematic nonlinear expansion (228) does not recover this damping at high orders. 


\subsection{Correlation function}

For the correlation function $G$, following the procedure of Sects. 11.2 and 12.1, we write

$\Delta^{2}(k ; D)=\Delta_{\mathrm{L}}^{2} g\left(t, \Delta_{\mathrm{L} 0}^{2}\right) \quad$ with $\quad t=D$.

Then, again introducing the Laplace transform with respect to $t$, inverting the series $\tilde{g}\left(s, \Delta_{\mathrm{L} 0}^{2}\right)=1 / s+.$. and substituting into $\partial \tilde{g} / \partial \Lambda$ gives the expansion:

$\frac{\partial \tilde{g}}{\partial \Lambda}=\frac{\mathrm{d} \Delta_{\mathrm{L} 0}^{2}}{\mathrm{~d} \Lambda}\left[\frac{3 \pi^{2}}{32} \tilde{g}^{3}-\frac{3 \pi^{2}\left(512+9 \pi^{2}\right)}{1024} \Delta_{\mathrm{L} 0}^{2} \tilde{g}^{5}+\cdots\right]$.

At order $p=0$ we have the linear correlation $\partial \tilde{g} / \partial \Lambda=0, \tilde{g}=$ $1 / s$ and $g(t)=1$. At order $p=1$ we obtain

$\frac{\partial \tilde{g}}{\partial \Delta_{\mathrm{L} 0}^{2}}=\frac{3 \pi^{2}}{32} \tilde{g}^{3}, \quad \tilde{g}^{(1)}(s)=\frac{1}{\sqrt{s^{2}-3 \pi^{2} \Delta_{\mathrm{L} 0}^{2} / 16}}$,

which gives

$g^{(1)}\left(t, \Delta_{\mathrm{L} 0}^{2}\right)=I_{0}\left(\frac{\sqrt{3} \pi}{4} \Delta_{\mathrm{L} 0} t\right)$,

where $I_{0}$ is the modified Bessel function of order 0 . Thus, as for the simple nonlinear scheme associated with the 2PI effective action method of Sect. 11.2, we obtain at order $p=1$ a nonlinear equal-time power $\Delta^{2}$ which shows an exponential growth in the nonlinear regime. Following the method leading to Eq. (234), we can actually integrate the nonlinear Eq. (237) at any order, using the scaling $\tilde{g}\left(s, \Delta_{\mathrm{L} 0}^{2}\right)=\tilde{\gamma}\left(s / \Delta_{\mathrm{L} 0}\right) / \Delta_{\mathrm{L} 0}$, which transforms Eq. (237) into an implicit equation giving $y=s / \Delta_{\mathrm{L} 0}$ as the integral of a rational function of $\tilde{\gamma}$. We can check that, at order $p=2$, we again have an exponential growth into the nonlinear regime (with oscillations because $\operatorname{Im}\left(y_{*}\right) \neq 0$ ).

\section{Weakly nonlinear scales}

In the previous sections, we have investigated the convergence properties of several expansion schemes that may be used to study gravitational clustering in the expanding Universe, applied to the case of the Zeldovich dynamics where exact results can be obtained. In this section, we complete this study by a brief description of the results obtained at one-loop order on weakly nonlinear scales. Indeed, an accurate prediction for the matter power spectrum on these scales is of great practical interest for several cosmological probes, such as baryonic acoustic oscillations (Eisenstein et al. 1998, 2005) and weak-lensing shear (Munshi et al. 2007). For the exact gravitational dynamics, the various expansion schemes must be compared with numerical simulations, which is not very convenient to evaluate their power. Therefore, it is interesting to check the accuracy and the behavior of these expansion methods against the Zeldovich dynamics.

\subsection{Linear expansion schemes}

We first consider "linear" expansion schemes, that is, methods that give rise to expansions in terms of the linear power spectrum, such as the standard perturbative expansions of Sect. 7 and the direct steepest-descent methods of Sect. 8. We focus on the equal-time power spectrum for the $\Lambda \mathrm{CDM}$ universe described in the first paragraph of Sect. 7. We used the CAMB Boltzmann code (Lewis et al. 2000) to obtain the linear power spectrum

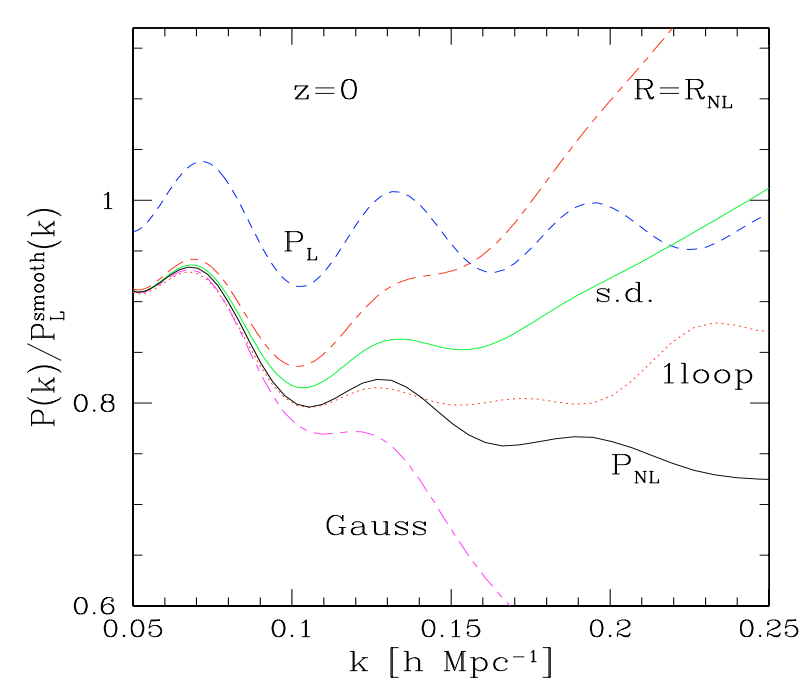

Fig. 20. The power spectrum $P(k)$ divided by a smooth linear power $P_{\mathrm{L}}^{\text {smooth }}$ at redshift $z=0$. We display the linear power $P_{\mathrm{L}}(k)$ (dashed line), the exact nonlinear power $P_{\mathrm{NL}}$ of Eq. (109), the standard 1-loop result (dotted line) of Eq. (201), and the steepest-descent result of Eq. (83) (upper solid line "s.d."). We also show the results obtained by adding a Gaussian factor to the standard perturbative result as in Eq. (159) (lower dot-dashed line) or by using the exact nonlinear response $R_{\mathrm{NL}}$ in Eq. (83) within the steepest-descent scheme (upper dashed line), as in Fig. 10.

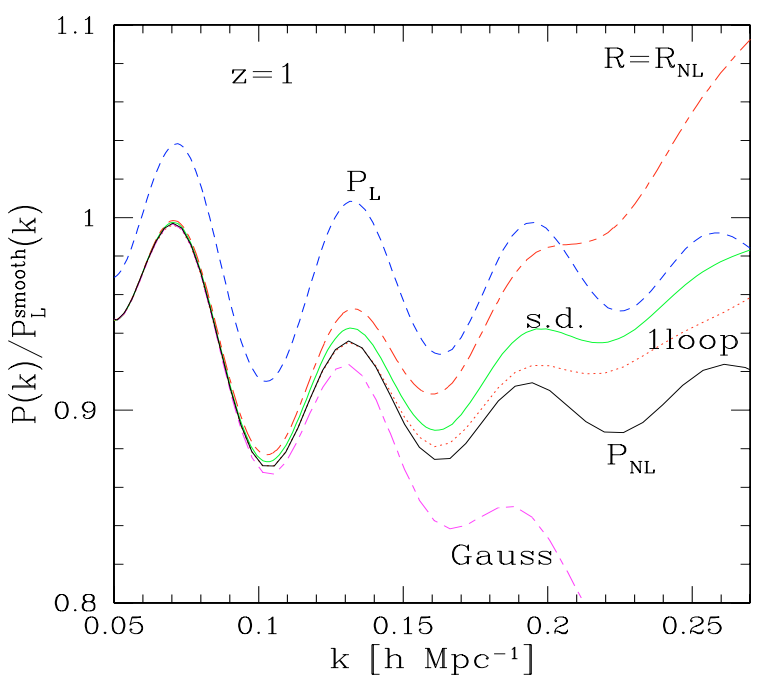

Fig. 21. The power spectrum $P(k)$ divided by a smooth linear power $P_{\mathrm{L}}^{\text {smooth }}$ as in Fig. 20, but at redshift $z=1$.

with the baryonic acoustic oscillations. In order to magnify the difference between various schemes, we show in Figs. 20 and 21 the nonlinear power divided by the linear power $P_{\mathrm{L}}^{\text {smooth }}$ associated to a smooth power spectrum without baryonic oscillations, taken from Eisenstein \& $\mathrm{Hu}$ (1998). We compared the results of various expansion schemes with the exact nonlinear power (solid line labeled $P_{\mathrm{NL}}$ ) obtained from the numerical integration of Eq. (109) for the $\Lambda \mathrm{CDM}$ power spectrum. First, we see in Fig. 20 that all schemes agree with the exact power up to $k \simeq 0.1 \mathrm{~h} \mathrm{Mpc}^{-1}$ at redshift $z=0$ and follow the departure from the linear power $P_{\mathrm{L}}$. On smaller scales, the various expansion schemes deviate from one another and from the exact 
nonlinear power. It is interesting to note that using the exact nonlinear response (133) within the steepest-descent scheme does not improve the agreement over the original steepest-descent scheme where we use the response function predicted at the same order. On the other hand, it appears that the standard oneloop expansion provides the best results at this order.

Following Eq. (159) and Fig. 5, we also consider the expansion defined from the standard perturbative series by factorizing a Gaussian decay:

$$
\begin{aligned}
P_{\text {Gauss. }}^{1 \text { loop }}(k) & =\mathrm{e}^{-D^{2} \omega^{2}}\left[P_{\mathrm{L}}+P_{22}+P_{13}+D^{2} \omega^{2} P_{\mathrm{L}}\right] \\
& =\mathrm{e}^{-D^{2} \omega^{2}}\left(P_{\mathrm{L}}+P_{22}\right) .
\end{aligned}
$$

In agreement with Sect. 7 and Crocce \& Scoccimarro (2006a), this gives a positive power whatever the shape of the linear power spectrum; however, Fig. 20 shows that this does not necessarily improve the accuracy as compared with the usual one-loop result (201). We show the power spectrum obtained at redshift $z=1$ in Fig. 21. Of course the various expansion schemes agree more closely on the same scales with the exact result, since we are closer to the linear regime. We can see that we recover the same behaviors as in Fig. 20.

The behavior of these various expansion schemes at higher orders was investigated in Sects. 7, 8. We found that no linear scheme provides a significant improvement over the standard perturbative expansion. Factorizing a Gaussian decaying term as in Eq. (240) seemed to give a small improvement in Fig. 5, but as discussed below Fig. 5, this is not very robust and would not apply to any power spectrum. On the other hand, Fig. 10 shows that even using the exact nonlinear response within the direct steepest-descent scheme does not generically provide a significant improvement either. Nevertheless, the most promising scheme within this framework is probably to combine a good ansatz for the response function with such expansions (see also Sect. 13.2 below). Then, one could hope to gain from the higher orders, while the imposed decay of the response function could tame the increasingly fast growth at higher orders obtained in the standard perturbative expansion. An application of such a strategy was presented in Crocce \& Scoccimarro (2007) for a $\Lambda$ CDM universe, and a significant improvement over the standard perturbative expansion was obtained up to two-loop order.

\subsection{Nonlinear expansion schemes}

Finally, we study in this section "nonlinear" expansion schemes, that is, methods that give rise to expansions in terms of the nonlinear two-point functions $R$ and $G$, such as the 2PI effective action method of Sects. 4.2 and 10. We show our results for the power spectrum (divided again by $P_{\mathrm{L}}^{\mathrm{smooth}}$ ) at redshifts $z=0,1$ in Figs. 22 and 23. First, we note that the 2 PI effective action result at one-loop order overestimates the power spectrum on weakly nonlinear scales. Both the standard one-loop result and the direct steepest-descent method actually work better in this range. This behavior can be traced back to the damping self-energy $\Sigma$. Indeed, since $\Sigma \propto R G$ at one-loop order (see Eq. (81)), the decay of the response and of the two-point correlation at high $k$ (shown in Figs. 16 and 18)) leads to a smaller $\Sigma(k)$ at high $k$ as compared with the $\Sigma_{0}$ obtained for the direct steepest-descent method. This in turns yields a response $R$ that is somewhat larger than for the steepest-descent method in the weakly nonlinear regime (but smaller at high $k$ where it decays) whence a twopoint correlation $G$ that is somewhat larger from Eq. (83). Of course, this slight overestimate of $R$ and underestimate of $\Sigma$ will be corrected by higher-order terms. For the Zeldovich dynamics,

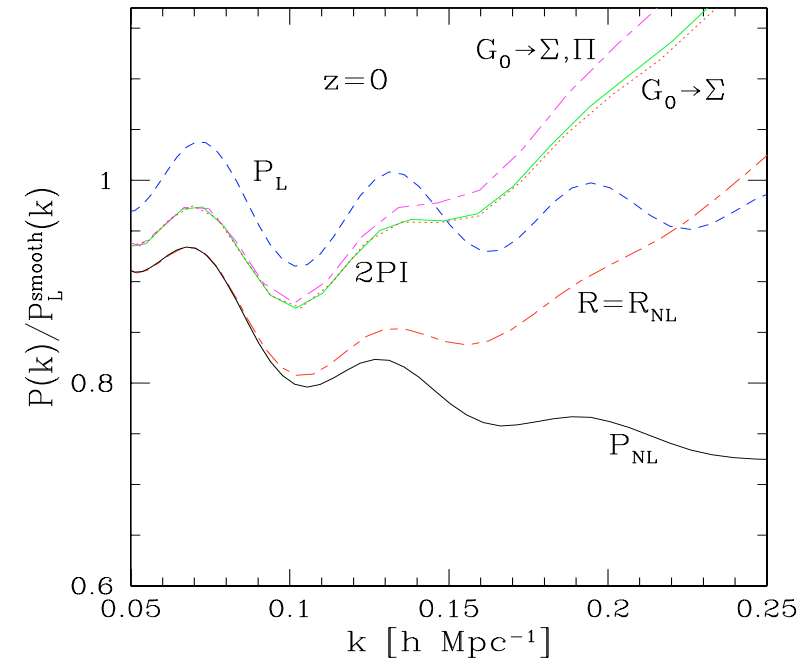

Fig. 22. The power spectrum $P(k)$ divided by a smooth linear power $P_{\mathrm{L}}^{\text {smooth }}$ at redshift $z=0$. We display the linear power $P_{\mathrm{L}}(k)$ (dashed line), the exact nonlinear power $P_{\mathrm{NL}}$ of Eq. (109), and the 2PI effective action result of Eq. (83) (upper solid line "2PI"). We also show the results obtained by using the exact nonlinear response $R_{\mathrm{NL}}$ in Eq. (83) within the 2PI scheme (lower dashed line), or by using the linear two-point correlation to compute both $\Sigma$ and $\Pi$ (upper dot-dashed line) or only $\Sigma$ (dotted line).

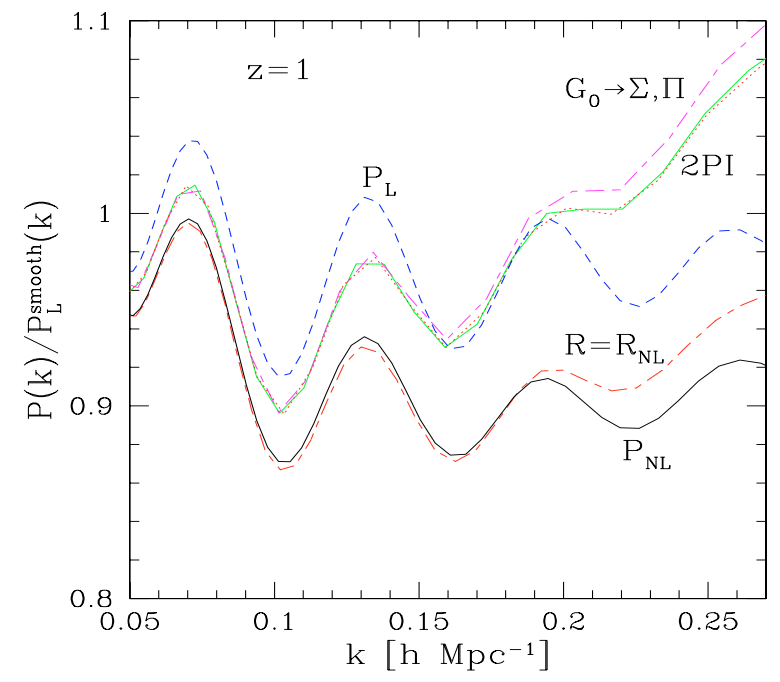

Fig. 23. The power spectrum $P(k)$ divided by a smooth linear power $P_{\mathrm{L}}^{\text {smooth }}$ as in Fig. 22, but at redshift $z=1$.

we know that $R$ and $\Sigma$ actually depend only on $\sigma_{v}$, so that terms that depend on other integrals over $P_{\mathrm{L} 0}(k)$ must cancel out in the full resummation. However, this discrepancy makes the one-loop 2PI effective action result insufficient for practical purposes (at least at the one-loop order).

We also display the results obtained when both the selfenergies $\Sigma$ and $\Pi$ are obtained from the linear correlation $G_{0}$ (so that only the response $R$ coupled to $\Sigma$ is obtained from nonlinear equations) and when only the self-energy $\Sigma$ is obtained from $G_{0}$ (so that the nonlinear systems for the pairs $\{\Sigma, R\}$ and $\{\Pi, G\}$ are decoupled). We see that these two methods give 
results very close to the original $2 \mathrm{PI}$ effective action prediction where all two-point functions $\{\Sigma, \Pi, R, G\}$ are coupled.

On the other hand, we also show the power spectrum obtained from the coupled Eqs. (82) and (83), when we use the exact nonlinear response (133). We can see that this significantly improves the agreement with the exact power spectrum, and the result is slightly better than the steepest-descent prediction shown in Fig. 20, but it is still a bit less accurate than the standard perturbative result at $z=0$. However, Fig. 23 shows that at $z=1$ the power obtained in this fashion is slightly more accurate than the standard perturbative result. Therefore, contrary to the case of the steepest-descent method studied in Sect. 13.1, using the exact nonlinear response improves the prediction for the two-point correlation and provides a scheme that can be competitive with the usual perturbative expansion on weakly nonlinear scales.

At higher orders, the nonlinear 2PI expansion scheme - especially if it uses the exact nonlinear response or a reliable ansatz (as for the lower dashed lines of Figs. 20-23) - may provide an even greater improvement as compared with the standard perturbative expansion. The analysis of Sects. 11, 12 showed that nonlinear schemes can give unwanted exponential growths at higher orders for both the response function and the power spectrum. However, this does not rule out a good convergence on weakly nonlinear scales. Nevertheless, this behavior and Figs. 20-23 suggest that the most promising scheme would be to combine a good ansatz for the response with such nonlinear expansions. As for the linear schemes of Sect. 13.1, one could hope to benefit from the higher orders, while the imposed decay of the response function could partly restrain their increasingly fast growth. This could make the improvement over the standard perturbative expansion even more significant at higher orders, but such a study is left for future work.

\section{Conclusion}

In this article we have applied to the Zeldovich dynamics various expansion schemes that may also be used for the gravitational dynamics in the expanding Universe. We derived the path-integral formalism that describes this system, starting either from the differential or the integral form of the equations of motion, and we obtained the relationship between the associated response functions. These response functions describe the response of the system to a small perturbation applied at any time and they also encode the memory of initial conditions. Next, we briefly described how to build various expansion schemes from these path integrals, such as large- $N$ expansions or running with a high- $k$ cutoff. All these results apply almost identically to the case of the gravitational dynamics.

Then, we have derived the exact nonlinear two-point functions associated with the Zeldovich dynamics, taking advantage of the well-known exact solution of the equations of motion. Whereas the equal-time nonlinear power decays as a power law in the highly nonlinear regime, different-time two-point functions, such as the response $R\left(k ; t_{1}, t_{2}\right)$ or the correlation $G\left(k ; t_{1}, t_{2}\right)$, show a Gaussian decay $\mathrm{e}^{-k^{2} \sigma_{v}^{2}\left(D_{1}-D_{2}\right)^{2} / 2}$, where $\sigma_{v}$ is the variance of the linear velocity. This damping is associated with the uniform random displacement of particles between different times by long wavelength modes. This leads to an effective decorrelation but it is not directly related to the matter clustering. In particular, $\sigma_{v}^{2}$ can be made very large, and even infinite, through low- $k$ divergences, without affecting the equal-time matter power spectrum. This also means that the matter power spectrum $P(k ; t)$ may still be very close to linear on scale $k$ even though two-point functions such as $R\left(k ; t_{1}, t_{2}\right)$ may have already shown large deviations from their linear values on the same scale at previous times $t_{2}<t_{1}<t$. Therefore, departures from linearity of different two-point functions are not necessarily related.

Next, we have studied the standard perturbative expansions for both the response $R$ and the logarithmic power $\Delta^{2}(k ; t) \propto$ $k^{3} P(k ; t)$, focussing on a power-law linear power spectrum $n=-2$ for the latter. The usual perturbative expansion being equivalent to a Taylor expansion it cannot capture the differenttime Gaussian decay of $R$ and $\Delta^{2}$, nor the equal-time power-law decay of $\Delta^{2}$, since it gives polynomial approximations of increasing order. In the spirit of Crocce \& Scoccimarro (2006a), we noticed that for the power $\Delta^{2}$, reorganizing the perturbative series by factorizing a simple Gaussian term $\mathrm{e}^{-D^{2} k^{2} \sigma_{v}^{2}}$ gives an expansion which looks better behaved, as all terms become positive and there is a Gaussian damping. However, this procedure only works for linear power-spectra such that the scales which govern $\sigma_{v}^{2}$ and $\Delta^{2}$ are close (as for typical $\Lambda$ CDM power-spectra).

Then, we have studied the steepest-descent method derived from a large- $N$ expansion. At first order $p=1$, there is some improvement for the response function, which remains bounded (as a cosine) instead of growing as $k^{2}$. (The same behavior is obtained for the gravitational dynamics Valageas 2007.) However, at higher orders the expansion worsens as it exhibits an exponential growth in the nonlinear regime. We showed that this could be cured by using Padé approximants, which remain bounded at all orders as a sum of cosines (they do not decay but after integration the oscillations should produce some effective damping). The power $\Delta^{2}$ displays an exponential growth at orders $p \geq 2$ for the plain steepest-descent method and a polynomial growth using the Padé approximants. As for the standard perturbative expansion, one can reorganize the series by factorizing a Gaussian term $\mathrm{e}^{-\left(D_{1}^{2}+D_{2}^{2}\right) k^{2} \sigma_{v}^{2} / 2}$ in the self-energy $\Pi$ that describes the generation of power by nonlinear interactions. This appears to give slightly better results than with the standard perturbative expansion, but this procedure obviously suffers from the same restrictions. Alternatively, one can factorize a Gaussian term such as $\mathrm{e}^{-\left(D_{1}^{2}-D_{2}^{2}\right) k^{2} \sigma_{v}^{2} / 2}$ into the self-energy $\Pi$, to reproduce the fact that the Gaussian damping must disappear for equal-time statistics. Then, one again obtains a polynomial growth into the highly nonlinear regime for the power $\Delta^{2}(k ; t)$, because of the contribution of mode couplings at recent times $t_{1} \simeq t_{2} \simeq t$. Therefore, none of these methods shows very satisfactory global convergence properties.

Next, we have discussed a high- $k$ resummation proposed by Crocce $\&$ Scoccimarro (2006b) to improve the behavior of such expansion schemes. We showed that the partial resummation involved in this procedure is equivalent to approximating the nonlinear equation of motion by a linear equation. Using the high- $k$ asymptotic of the coupling kernel, as in Crocce \& Scoccimarro (2006b), we derived the explicit expression of the nonlinear density field $\delta(\boldsymbol{x}, t)$ associated with these approximations. Then, both the nonlinear response $R$ and power $\Delta^{2}$ are equal to their linear counterpart multiplied by the same differenttime Gaussian decay. Thus, these approximations manage to capture the different-time Gaussian decay (associated with the advection by long wavelengths) but they fail to capture the equaltime properties of the system. A close analysis of this procedure shows that the underlying assumptions are not valid because one cannot define a high- $k$ limit in a simple manner. (For a linear power spectrum with $-3<n<-1$ at high $k$ the nonlinear power at wavenumber $k$ is generated by the highly nonlinear couplings of modes $k^{\prime} \gg k$ instead of being produced by small 
wavenumbers restricted to some finite range $k^{\prime}<\Lambda$.) The same caveats should apply to the gravitational dynamics, although it is not totally obvious whether the Gaussian decay at different times is exact in this case (but this is not necessarily important for practical purposes).

Then, we have turned to nonlinear schemes, that is, expansions over powers of nonlinear two-point functions, such as the 2PI effective action method built from a large- $N$ expansion. At one-loop order $(p=1)$, we obtain damped oscillations (with a power-law decay) for the two-time response function and power $\Delta^{2}\left(k ; t_{1}, t_{2}\right)$, but the equal-time power still grows on small scales. (One obtains the same behavior for the gravitational dynamics, Valageas 2007). Then, from the exact two-point functions we have built simple nonlinear expansion schemes that are similar to the 2PI effective action expansion, but that can be more easily handled analytically. We recover damped oscillations for the response function at order $p=1$, but we find an exponential growth at higher orders; the equal-time power also shows an exponential growth. Next, we investigated simple nonlinear expansion schemes associated with the evolution of the system with a high- $k$ cutoff $\Lambda$. We find a similar behavior as we again obtain damped oscillations at order $p=1$ and exponential growth at higher orders for the response function; the equal-time power also exhibits an exponential growth. To recover the Gaussian decay from this expansion at order $p=1$, one must introduce some further approximations, as in Matarrese \& Pietroni (2007a,b), which are correct at this order but which do not give a systematic procedure. Therefore, it appears that at high orders $p \geq 2$ nonlinear methods do not fare much better than linear schemes.

Finally, we have studied the quantitative predictions of these various schemes at one-loop order on weakly nonlinear scales for the equal-time matter power spectrum. All linear expansions agree with the exact results on quasi-linear scales $(k<$ $0.1 \mathrm{~h} \mathrm{Mpc}^{-1}$ at $z=0$ ) where there is already a deviation of $10 \%$ from linear theory. On smaller scales they depart from each other and the standard perturbation theory actually works best for the case studied in this article. Moreover, factorizing a Gaussian damping factor or using the exact response function does not improve the predictions obtained within this framework. On the other hand, the nonlinear 2PI effective action method overestimates the nonlinear power spectrum on these scales because of the nonlinear feedback involved by the coupled system obeyed by the response $R$. However, using the exact nonlinear response within this method now improves the agreement with the exact result and can be competitive with the standard perturbation expansion (but this depends on the exact shape of the linear power spectrum as in our case it is slightly better at $z=1$ but slightly worse at $z=0$ ).

Since the equations of motion associated with the gravitational and the Zeldovich dynamics are very close we can expect these results to apply to the gravitational case. (This is the case at one-loop order as shown by the comparison with Valageas, 2007.) We have found that none of these schemes shows good global convergence properties at high orders. Indeed, they all lead to polynomial or exponential growth into the nonlinear regime for the response function, except for the use of Padé approximants which gives bounded response functions with fast oscillations in the highly nonlinear regime. On the other hand, nonlinear schemes manage to reproduce the damping at one-loop order $p=1$ but fail at higher orders. Next, no scheme manages to recover the power-law damping of the nonlinear matter power spectrum. They either display increasing growth at higher order or a Gaussian decay which is also somewhat artificial.
Nevertheless, an expansion may still be very useful on weakly nonlinear scales even if it converges badly (or even diverges) on highly nonlinear scales. There, we found that the best methods seem to be the standard perturbation theory or a nonlinear expansion where one uses the exact nonlinear response (with the Gaussian decay). These results are somewhat disappointing, since it appears to be difficult to build systematic expansion schemes that significantly improve over the standard expansion. One may still obtain some improvement as in Crocce \& Scoccimarro (2007) or Matarrese \& Pietroni (2007a,b), but this requires some additional ingredients, such as the use of an ansatz, that shows a Gaussian decay, for the response function and in some cases for other two-point functions as well. On the other hand, the use of several expansion schemes can be of interest by itself, since they should be accurate at least over the range where they all agree. This allows one to obtain an estimate of their range of validity without the need to perform numerical simulations.

In order to make progress, it appears that it may be advantageous for observational purposes to be guided by the expected behavior of two-point functions and to combine such systematic expansions with reasonable ansatze (e.g. Crocce \& Scoccimarro 2007). From a theoretical perspective, one may also look for different approaches. For instance, one could try to work directly with the Vlasov equation (Valageas 2004). However, this would make the computations significantly more difficult, and it is not clear whether it is not more efficient to stick to the hydrodynamical approach and to simply compute higher order terms, especially if one is mostly interested in weakly nonlinear scales. On the other hand, one may consider simpler effective dynamics that attempt to go beyond shell crossing (based for instance on a Schroedinger equation, Widrow \& Kaiser 1993).

\section{References}

Bender, C. M., \& Orszag, S. A. 1978, Advanced mathematical methods for scientists and engineers (McGraw-Hill Book Co.)

Berges, J. 2002, Nucl. Phys. A, 699, 847

Bernardeau, F., Colombi, S., Gaztanaga, E., \& Scoccimarro, R. 2002, Phys. Rep., 367,1

Bouchaud, J.-P., Cugliandolo, L., Kurchan, J., \& Mezard, M. 1996, Physica A, 226,243

Crocce, M., \& Scoccimarro, R. 2006a, Phys. Rev. D, 73, 063519

Crocce, M., \& Scoccimarro, R. 2006b, Phys. Rev. D, 73, 063520

Crocce, M., \& Scoccimarro, R. 2007 [arXiv: 0704 .2783]

Crocce, M., Pueblas, S., \& Scoccimarro, R. 2006, MNRAS, 373, 369

Cvitanovic, P., Lautrup, B., \& Pearson, R. B. 1978, Phys. Rev. D, 18, 1939

Doherty, J. P., Moore, M. A., Kim, J., \& Bray, A. J. 1994, Phys. Rev. Lett., 72, 2041

Eisenstein, D. J., \& Hu, W. 1998, ApJ, 496, 605

Eisenstein, D. J., Hu, W., \& Tegmark, M. 1998, ApJ, 504, L57

Eisenstein, D. J., Zehavi, Idit, Hogg, D. W., et al. 2005, ApJ, 633, 560

Goroff, M. H., Grinstein, B., Rey, S.-J., \& Wise, M. B. 1986, ApJ, 311, 6

Gurbatov, S. N., Saichev, A. I., \& Shandarin, S. F. 1989, MNRAS, 236, 385

Jain, B., \& Bertschinger, E. 1994, ApJ, 431, 495

Jain, B., \& Bertschinger, E. 1996, ApJ, 456, 43

Kraichnan, R. 1961, J. Fluid Mech., 7, 124

Lewis, A., Challinor, A., \& Lasenby, A. 2000, ApJ, 538, 473

McDonald, P. 2007, Phys. Rev. D, 75, 043514

Martin, P. C., Siggia, E. D., \& Rose, H. A. 1973, Phys. Rev. A, 8, 423

Massey, R., Rhodes, J., Leauthaud, A., et al. 2007, ApJ, accepted [arXiv: astro-ph/0701480]

Matarrese, S., \& Pietroni, M. 2007a [arXiv: astro-ph/0702653]

Matarrese, S., \& Pietroni, M. 2007b [arXiv: astro-ph/0703563]

Mou, C. Y., \& Weichman, P. 1993, Phys. Rev. Lett., 70, 1101

Munshi, D., Valageas, P., Van Waerbeke, L., \& Heavens, A. 2007, Phys. Rep., submitted [arXiv: astro-ph/0612667] 
Oukbir, J., \& Blanchard, A. 1992, A\&A, 262, L21

Peebles, P. J. E. 1980, The large scale structure of the universe (Princeton University Press)

Peebles, P. J. E. 1982, ApJ, 263, L1

Phythian, R. 1977, J. Phys. A, 10, 777

Schneider, P., \& Bartelmann, M. 1995, MNRAS, 273, 475

Scoccimarro, R. 2000, Ann. N.Y. Acad. Sci., 927, 13 [arXiv: astro-ph/0008277]

Scoccimarro, R., \& Frieman, J. 1996a, ApJS, 105, 37

Scoccimarro, R., \& Frieman, J. 1996b, ApJ, 473, 620

Seljak, U. 2000, MNRAS, 318, 203

Seljak, U., \& Zaldarriaga, M. 1996, ApJ, 469, 437

Shandarin, S. F., \& Zeldovich, Y. B. 1989, Rev. Mod. Phys., 61, 185
Smith, R. E., Peacock, J. A., Jenkins, A., et al. 2003, MNRAS, 341, 1311

Taylor, A. N., \& Hamilton, A. J. S. 1996, MNRAS, 282, 767

Valageas, P. 2001, A\&A, 379, 8

Valageas, P. 2002, A\&A, 382, 477

Valageas, P. 2004, A\&A, 421, 23

Valageas, P. 2007, A\&A, 465, 725

Vishniac, E. T. 1983, MNRAS, 203, 345

Widrow, L. M., \& Kaiser, N. 1993, ApJ, 416, L71

Younger, J. D., Bahcall, N. A., \& Bode, P. 2005, ApJ, 622, 1

Zeldovich, Y. B. 1970, A\&A, 5, 84

Zinn-Justin, J. 1989, Quantum field theory and critical phenomena (Oxford: Clarendon Press) 